\title{
Coordinated Multi-Point Transmission for Interference Mitigation in Cellular Distributed Antenna Systems
}

by

\section{Talha Ahmad, B.Eng.}

A thesis submitted to the Faculty of Graduate and Postdoctoral Affairs in partial fulfillment of the requirements for the degree of Master of Applied Science in Electrical and Computer Engineering

Ottawa-Carleton Institute for Electrical and Computer Engineering (OCIECE) Department of Systems and Computer Engineering

Carleton University

Ottawa, Ontario, Canada

June 2011

Copyright (C)

Talha Ahmad, 2011 


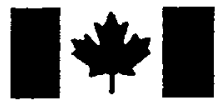

Library and Archives

Canada

Published Heritage

Branch

395 Wellington Street

Ottawa ON K1A 0N4

Canada
Bibliothèque et

Archives Canada

Direction du

Patrimoine de l'édition

395 , rue Wellington

Ottawa ON K1A ON4

Canada
Your file Votre référence
ISBN: 978-0-494-83061-1
Our file Notre référence
ISBN: $978-0-494-83061-1$
NOTICE:

The author has granted a nonexclusive license allowing Library and Archives Canada to reproduce, publish, archive, preserve, conserve, communicate to the public by telecommunication or on the Internet, loan, distribute and sell theses worldwide, for commercial or noncommercial purposes, in microform, paper, electronic and/or any other formats.

The author retains copyright ownership and moral rights in this thesis. Neither the thesis nor substantial extracts from it may be printed or otherwise reproduced without the author's permission.
AVIS:

L'auteur a accordé une licence non exclusive permettant à la Bibliothèque et Archives Canada de reproduire, publier, archiver, sauvegarder, conserver, transmettre au public par télécommunication ou par l'Internet, prêter, distribuer et vendre des thèses partout dans le monde, à des fins commerciales ou autres, sur support microforme, papier, électronique et/ou autres formats.

L'auteur conserve la propriété du droit d'auteur et des droits moraux qui protège cette thèse. Ni la thèse ni des extraits substantiels de celle-ci ne doivent être imprimés ou autrement reproduits sans son autorisation.
In compliance with the Canadian Privacy Act some supporting forms may have been removed from this thesis.

While these forms may be included in the document page count, their removal does not represent any loss of content from the thesis.
Conformément à la loi canadienne sur la protection de la vie privée, quelques formulaires secondaires ont été enlevés de cette thèse.

Bien que ces formulaires aient inclus dans la pagination, il n'y aura aucun contenu manquant. 
The undersigned recommend to the Faculty of Graduate and Postdoctoral Affairs acceptance of the thesis

\title{
Coordinated Multi-Point Transmission for Interference Mitigation in Cellular Distributed Antenna Systems
}

\author{
Subminted by \\ Talha Alunad, B.Eng. \\ in partial fulfillment of the requirements for the degree of \\ Master of Applied Science in Electrical and Computer Engineering
}

Thesis Supervisor, Dr. Halim Yanıö̈meroğlu

Chair, Department of Systcms and Computer Engineering, Dr. Howard Schwartz

Carleton University

2011 


\section{In the name of God, most Gracious, most Merciful}

"Read! In the name of your Lord Who has created (all that exists) - created man

from a clot. Read! And your Lord is the Most Generous - He Who has taught (the use of) the pen. He has taught man that which he did not know."

The Holy Qur'an, Chapter 96, Verses 1-5 


\section{Abstract}

Distributed antenna systems (DASs) have been shown to improve the coverage and increase the capacity in cellular networks by reducing the access distance to user terminals (UTs) and by attaining macrodiversity gains. However, the conventional DASs do not inherently mitigate inter-cell interference. In this thesis, coordinated multi-point transmission schemes are developed for interference mitigation in the downlink of a cellular DAS.

The thesis is comprised of two parts. In the first part, two precoding schemes are developed, which enable coordinated transmission from multiple distributed antenna ports in a cellular DAS with a total power constraint. The goal is to serve multiple UTs in a particular resource block in each cell, while mitigating intra-cell and intercell interference. Simulation is used to show the performance gains attained by the proposed DAS schemes as compared to their co-located antenna system counterparts, and by centralized multi-cell processing as compared to single-cell processing.

In the second part, the joint selection of the ports and the corresponding beam steering coefficients that maximize the minimum signal-to-interference-plus-noise ratio of the UTs in a coordinated multi-cell DAS, in which the transmit power of each port is fixed, is considered. This problem is NP-hard. To circumvent this difficulty, a two-stage polynomial-complexity technique that relies on semidefinite relaxation and Gaussian randomization is developed. The performance of the proposed technique is shown to be comparable to that of exhaustive search. Additionally, it is demonstrated that proper port selection yields significant power savings in the cellular network. 


\section{Acknowledgements}

I begin by praising God, the Creator and Sustainer of all the worlds.

I would like to sincerely thank my supervisor, Professor Halim Yanıkömeroḡlu, for his support, guidance, and encouragement throughout the course of my master's program. I am also grateful to him for giving me the opportunity to be a part of a knowledgeable, productive, and tightly-knit research group.

My sincere gratitude to Dr. Gary Boudreau (Ericsson Canada), whose insightful advice greatly enhanced the quality of my research.

A very special thanks to Dr. Saad Al-Ahmadi, Dr. Ramy Gohary, and Akram Salem Bin Sediq, whose mentorship and influence significantly accelerated my learning and productivity.

On the personal side, I wish to thank my parents, grandparents, aunts, uncles, and cousins for their unconditional love, incomparable upbringing, and support of my academic aspirations.

I wish to thank my colleagues and friends, Furkan Alaca, Dr. Muhammad Aljuaid, Imran Ansari, Tamer Beitelmal, Dr. Gürhan Bulu, Dr. Ghassan Dahman, Soumitra Dixit, Dr. Petar Djukic, Heba Eid, Yaser Fouad, Arshdeep Kahlon, Kevin Luo, Mahmudur Rahman, Rozita Rashtchi, Dr. Mohamed Rashad Salem, Alireza Sharifian, Dr. Daniel Calabuig Soler, Dr. Sebastian Szyszkowicz, Aizaz Chaudhry, Tariq Shehata, Meraj Siddiqui, Nazia Ahmad, Mohammad Aslam Malik, Rajab Legnain, Amar Farouk Merah, Rami Sabouni, Shafiqul Islam, Muhammad Ajmal Khan, Dany David, and Dr. Laurence Smith for making my experience an enjoyable one. 


\section{Contents}

$\begin{array}{ll}\text { Abstract } & \text { ii }\end{array}$

$\begin{array}{ll}\text { Acknowledegements } & \text { iii }\end{array}$

Contents $\quad$ iv

List of Figures $\quad$ vi

List of Tables $\quad$ vii

Nomenclature viii

1 Introduction $\quad \mathbf{1}$

1.1 Cellular Networks . . . . . . . . . . . . . . . . . . . 1

1.2 Distributed Antenna Systems . . . . . . . . . . . . . . . . 2

1.3 Coordinated Multi-Point Transmission and Reception . . . . . . . . . 2

1.4 Thesis Contributions and Organization ............. 3

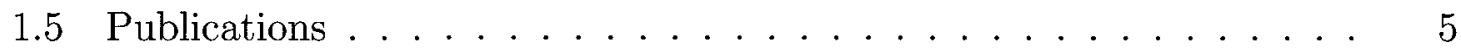

2 Background $\quad 7$

2.1 Related Works on Distributed Antenna Systems . . . . . . . . . . 7

2.2 Related Works on CoMP . . . . . . . . . . . . . . . 8

2.3 Information-Theoretic Background . . . . . . . . . . . . 9

3 Coordinated Multi-Point Downlink Transmission Schemes 11

3.1 Related Literature . . . . . . . . . . . . . . . . . . 11

3.2 Background: Basic Linear Algebra . . . . . . . . . . . . . . . . 12

3.2.1 Eigenvectors and Eigenvalues . . . . . . . . . . . . . 13

3.2 .2 Null Space of a Matrix . . . . . . . . . . . . . . . . . 13

3.2 .3 Rank of a Matrix ................. . . . 13

3.2.4 Singular Value Decomposition . . . . . . . . . . . . . 14

3.3 Single-Cell Processing . . . . . . . . . . . . . . . . . . . . . . . . . . . . . . .

3.3.1 System Model . . . . . . . . . . . . . . . . . . 14

3.3.2 DAS Block Diagonalization ............. . 19

3.3.3 DAS Zero-Forcing Dirty Paper Coding . . . . . . . . . . 23

3.3.4 An Exemplary Configuration. . . . . . . . . . . 25

3.4 Centralized Multi-Cell Processing . . . . . . . . . . . . . 27 
3.4.1 System Model . . . . . . . . . . . . . . . . . . . 28

3.4.2 DAS Block Diagonalization . . . . . . . . . . . . . . 29

3.4.3 DAS Zero-Forcing Dirty Paper Coding . . . . . . . . . . . 31

3.5 Performance Evaluation . . . . . . . . . . . . . . . . . 32

3.5.1 Single-Cell System (Interference-Free Environment) . . . . . . 33

3.5.2 Multi-Cell System with Single-Cell Processing . . . . . . . . . 41

3.5.3 Multi-Cell System with Centralized Processing . . . . . . . . 48

4 Coordinated Max-Min Fair Multi-Cell Port Selection and Beam Steering Optimization using Semidefinite Relaxation $\quad 53$

4.1 Related Literature . . . . . . . . . . . . . . . . . . . . 53

4.2 Background: Mathematical Optimization . . . . . . . . . . 54

4.2.1 Convex Optimization Problem . . . . . . . . . . . 55

4.2 .2 Semidefinite Programming . . . . . . . . . . . . 55

4.2.3 Relaxation of an Optimization Problem . . . . . . . . . . 55

4.3 System Model . . . . . . . . . . . . . . . . . . . . . 56

4.4 Problem Statement and Proposed Solution . . . . . . . . . . . 58

4.5 Coordinated Multi-Cell Port Selection . . . . . . . . . . . . 58

4.5.1 Positive Semidefinite Relaxation . . . . . . . . . . . . . . 61

4.5.2 Randomization for Coordinated Port Selection . . . . . . . . 64

4.6 Coordinated Beam Steering Optimization . . . . . . . . . . . . 66

4.7 A Two-Stage Approach to obtain an Approximate Solution to the Joint Optimization Problem . . . . . . . . . . . . . . 71

4.8 Complexity Analysis . . . . . . . . . . . . . . . . . . . 71

4.8.1 Computational Complexity of the First Stage . . . . . . . 72

4.8.2 Computational Complexity of the Second Stage . . . . . . . 73

4.8.3 Computational Complexity of the Two-Stage Approach . . . . 73

4.9 Performance Evaluation . . . . . . . . . . . . . . . . 74

5 Conclusions and Future Work 93

5.1 Summary . . . . . . . . . . . . . . . . . . 93

5.2 Contributions . . . . . . . . . . . . . . . . . . . . . 94

5.3 Future Work . . . . . . . . . . . . . . . . . . . . . . 94

$\begin{array}{ll}\text { Appendices } & 96\end{array}$

$\begin{array}{lr}\text { A Proofs and Derivations for Chapter 4 } & 97\end{array}$

A.1 Derivation of the matrix notation in $(4.7) \ldots \ldots \ldots \ldots$

A.2 Proof for Lemma $4.1 \ldots \ldots \ldots$

$\begin{array}{lr}\text { References } & 99\end{array}$ 


\section{List of Figures}

3.1 A single DAS cell with $K=3$ UTs per RB and $L=7$ ports, and an illustration of the general signal model. In this example, without loss of generality, $\mathcal{A}_{m}=\mathcal{S}_{m}=\{1,2, \ldots, 7\} \ldots \ldots \ldots$

3.2 An exemplary single-cell DAS configuration with $K=3$ UTs per RB and $L=7$ ports.

3.3 A comparison between the ergodic and outage aggregate cell spectral efficiencies per RB achieved by DAS BD and DAS ZF-DPC and those achieved by their CAS counterparts in a single-cell system that operates in an interference-free environment. $N_{t}=2$ and $\left|\mathcal{C}_{k m}\right|=3, \forall k$. .

3.4 A comparison between the ergodic and outage aggregate cell spectral efficiencies per RB achieved by DAS BD and DAS ZF-DPC and those achieved by their CAS counterparts in a single-cell system that operates in an interference-free environment. $N_{t}=3$ and $\left|\mathcal{C}_{k m}\right|=3, \forall k$. .

3.5 A comparison between the ergodic and outage aggregate cell spectral efficiencies per RB achieved by DAS BD and DAS ZF-DPC and those achieved by their CAS counterparts in a single-cell system that operates in an interference-free environment. $N_{t}=2$ and $\left|\mathcal{C}_{k m}\right|=7, \forall k$. .

3.6 A comparison between the aggregate cell spectral efficiencies per RB achieved by DAS BD and DAS ZF-DPC and those achieved by their CAS counterparts for different values of $N_{t}$ in a single-cell system that operates in an interference-free environment. $\left|\mathcal{C}_{k m}\right|=3, \forall k . \ldots$.

3.7 A comparison between the aggregate cell spectral efficiencies per RB achieved by DAS BD and DAS ZF-DPC for different values of $\left|\mathcal{C}_{k m}\right|$ in a single-cell system that operates in an interference-free environment.

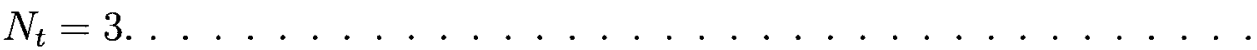

3.8 A comparison between the aggregate cell spectral efficiencies per RB achieved by DAS BD and DAS ZF-DPC, and those achieved by their CAS counterparts for different UT locations in a single-cell system that operates in an interference-free environment. $N_{t}=2$ and $\left|\mathcal{C}_{k m}\right|=3, \forall k .41$

3.9 A seven-cell DAS with single-cell processing, $K=3$ UTs per RB, and $L=7$ ports per cell. . . . . . . . . . . . . .

3.10 A comparison between the ergodic and outage aggregate cell spectral efficiencies per RB achieved by DAS BD and DAS ZF-DPC and those achieved by their CAS counterparts for a cell that operates in an intercell interference environment. $N_{t}=2$ and $\left|\mathcal{C}_{k m}\right|=3, \forall k \ldots \ldots$ 
3.11 A comparison between the ergodic and outage aggregate cell spectral efficiencies per RB achieved by DAS BD and DAS ZF-DPC and those achieved by their CAS counterparts for a cell that operates in an intercell interference environment. $N_{t}=3$ and $\left|\mathcal{C}_{k m}\right|=3, \forall k$. . . . . .

3.12 A comparison between the ergodic and outage aggregate cell spectral efficiencies per RB achieved by DAS BD and DAS ZF-DPC and those achieved by their CAS counterparts for a cell that operates in an intercell interference environment. $N_{t}=2$ and $\left|\mathcal{C}_{k m}\right|=7, \forall k \ldots \ldots$.

3.13 A comparison between the aggregate cell spectral efficiencies per RB achieved by DAS BD and DAS ZF-DPC for different values of $\left|\mathcal{C}_{k m}\right|$ for a cell that operates in an inter-cell interference environment. $N_{t}=3.47$

3.14 A seven-cell DAS with centralized multi-cell processing, $K=3$ UTs per RB, and $L=7$ ports per cell. . . . . . . . . . . . .

3.15 A comparison between the ergodic and outage average aggregate spectral efficiency per cell per RB achieved by DAS BD and DAS ZF-DPC with centralized multi-cell processing and with single-cell processing for a seven-cell cluster. $N_{t}=2$ and $\left|\mathcal{C}_{k}\right|=3, \forall k \ldots \ldots \ldots$

3.16 A comparison between the ergodic and outage average aggregate spectral efficiency per cell per RB achieved by DAS BD and DAS ZF-DPC with centralized multi-cell processing and with single-cell processing for a seven-cell cluster. $N_{t}=3$ and $\left|\mathcal{C}_{k}\right|=3, \forall k \ldots \ldots \ldots$

4.1 A seven-cell DAS cluster with seven ports per cell. . . . . . . . . . .

4.2 A comparison between the largest minimum SINR achieved by port selection with exhaustive search and with the technique proposed in the first stage, and that achieved by the one-port (without coordination) and all-port strategies for a two-cell cluster in the SMa scenario. . . .

4.3 A comparison between the largest minimum spectral efficiency achieved by port selection with exhaustive search and with the technique proposed in the first stage, and that achieved by the one-port (without coordination) and all-port strategies for a two-cell cluster in the SMa scenario. . . . . . . . . . . . . . . . . . . . .

4.4 A comparison between the largest minimum SINR achieved by the port selection technique proposed in the first stage, and that achieved by the one-port (without coordination) and all-port strategies for a seven-cell cluster in the SMa scenario. . . . . . . . . . . . . . . . . . .

4.5 A comparison between the largest minimum spectral efficiency achieved by the port selection technique proposed in the first stage, and that achieved by the one-port (without coordination) and all-port strategies for a seven-cell cluster in the SMa scenario. . . . . . . . . . . . .

4.6 The distribution of the number of ports activated per cell by the port selection technique proposed in the first stage for a seven-cell cluster in the SMa scenario. . . . . . . . . . . . . . . . . . 
4.7 The average number of ports activated per cell by the port selection technique proposed in the first stage for a seven-cell cluster in the SMa scenario. . . . . . . . . . . . . . . . . . . .

4.8 A comparison between the average spectral efficiency per cell achieved by the port selection technique proposed in the first stage, by the singleport (without coordination) and all-port transmission strategies, and by the CAS for a seven-cell cluster in the SMa scenario. . . . . . . . .

4.9 A comparison between the largest minimum SINR achieved by the port selection technique proposed in the first stage, and that achieved by the one-port (without coordination) and all-port strategies for a seven-cell cluster in the UMa scenario. . . . . . . . . . . . . . . . . . . .

4.10 A comparison between the largest minimum spectral efficiency achieved by the port selection technique proposed in the first stage, and that achieved by the one-port (without coordination) and all-port strategies for a seven-cell cluster in the UMa scenario. . . . . . . . . . . . . . .

4.11 The distribution of the number of ports activated per cell by the port selection technique proposed in the first stage for a seven-cell cluster in the UMa scenario. . . . . . . . . . . . . . . . . . . .

4.12 The average number of ports activated per cell by the selection technique proposed in the first stage for a seven-cell cluster in the UMa scenario. . . . . . . . . . . . . . . . . .

4.13 A comparison between the average spectral efficiency per cell achieved by the port selection technique proposed in the first stage, by the singleport (without coordination) and all-port transmission strategies, and by the CAS for a seven-cell cluster in the UMa scenario. . . . . . . .

4.14 A comparison between the largest minimum SINR achieved by the computationally-efficient two-stage approach proposed herein, an exhaustivesearch based close-to-optimal solution, and the port selection technique in the first stage for a two-cell cluster in the SMa scenario. . . . . . . .

4.15 A comparison between the largest minimum spectral efficiency achieved by the computationally-efficient two-stage approach proposed herein, an exhaustive-search based close-to-optimal solution, and the port selection technique in the first stage for a two-cell cluster in the SMa scenario. . . . . . . . . . . . . . . . 


\section{List of Tables}

3.1 System and simulation parameters used for the cellular DAS architecture 33

4.1 Algorithm 1 - Generating a close-to-optimal set of port states . . . . 66

4.2 Algorithm 2 - Generating close-to-optimal beam steering coefficients . 70

4.3 Complexity of the proposed techniques and the corresponding exhaustive search ..................... . . . 74

4.4 System parameters used to simulate the cellular DAS architecture . . 74

4.5 IMT-Advanced scenario parameters used to simulate the cellular DAS 75

4.6 Channel gains between the ports and the UTs in each cell $\left(\times 10^{-4}\right) \cdot 92$ 


\section{Nomenclature}

\section{Acronyms}

\begin{tabular}{|c|c|}
\hline Acronym & Meaning \\
\hline $3 \mathrm{GPP}$ & 3rd Generation Partnership Project \\
\hline $\mathrm{BC}$ & Broadcast channel \\
\hline $\mathrm{BD}$ & Block diagonalization \\
\hline $\mathrm{BS}$ & Base station \\
\hline CAS & Co-located antenna system \\
\hline CDMA & Code division multiple access \\
\hline CoMP & Coordinated multi-point \\
\hline CSI & Channel state information \\
\hline DAS & Distributed antenna system \\
\hline $\mathrm{DPC}$ & Dirty paper coding \\
\hline $\mathrm{IC}$ & Interference channel \\
\hline ICI & Inter-cell interference \\
\hline LTE & Long Term Evolution \\
\hline IMT & International Mobile Telecommunications \\
\hline $\mathrm{MAC}$ & Medium access control \\
\hline MIMO & Multiple-input multiple-output \\
\hline NLoS & Non-line-of-sight \\
\hline NP-hard & Non-deterministic polynomial-time hard \\
\hline OFDM & Orthogonal frequency division multiplexing \\
\hline
\end{tabular}


OFDMA Orthogonal frequency division multiple access

PSD Positive semidefinite

RB Resource block

SDP Semidefinite program

SDR Semidefinite relaxation

SINR Signal-to-interference-plus-noise ratio

SMa Suburban macro-cell

SNR Signal-to-noise ratio

SVD Singular value decomposition

UMa Urban macro-cell

UT User terminal

ZFBF Zero-forcing beamforming

ZF-DPC Zero-forcing dirty paper coding 


\section{Mathematical Operators and Symbols}

\begin{tabular}{|c|c|}
\hline Symbol & Meaning \\
\hline$(\cdot)^{*}$ & Complex conjugate \\
\hline$(\cdot)^{T}$ & Transpose of the vector or matrix argument \\
\hline$(\cdot)^{H}$ & Hermitian of the vector or matrix argument \\
\hline $\boldsymbol{I}_{N}$ & $N \times N$ identity matrix \\
\hline $\mathbf{0}_{M \times N}$ & $M \times N$ all zero-matrix \\
\hline $\mathbb{R}^{n \times m}$ & Space of $n \times m$ real matrices \\
\hline $\mathbb{C}^{n \times m}$ & Space of $n \times m$ complex matrices \\
\hline$|\cdot|$ & $\begin{array}{l}\text { Absolute value of the scalar argument, determinant of the matrix } \\
\text { argument, or cardinality of the set argument }\end{array}$ \\
\hline$\|\cdot\|_{2}$ & Frobenius norm of the matrix argument \\
\hline$E\{\cdot\}$ & Expected value \\
\hline$P\{\mathcal{E}\}$ & Probability of event $\mathcal{E}$ occurring \\
\hline $\operatorname{Tr}(\cdot)$ & Trace of the matrix argument \\
\hline $\operatorname{rank}(\cdot)$ & Rank of the matrix argument \\
\hline $\operatorname{diag}(\cdot)$ & Vector comprised of the diagonal elements of the matrix argument \\
\hline$\oplus$ & Direct sum of matrices \\
\hline$\{\bar{\cdot}\}$ & Set complement \\
\hline$\{\cdot\} \backslash\{\cdot\}$ & Set difference \\
\hline $\operatorname{sgn}(\cdot)$ & Element-wise signum function \\
\hline
\end{tabular}




\section{Chapter 1}

\section{Introduction}

\subsection{Cellular Networks}

Cellular networks gained commercial momentum during the 1990s as a convenient means of voice communication. The primary usage of these networks has since shifted toward data communication, and it is expected that data-intensive applications, such as mobile Internet and multimedia services, will consume most of the resources in future cellular networks.

In a cellular network, a geographical area is tessellated into smaller regions called cells. A base station (BS) is located in each cell and it provides wireless services to mobile user terminals (UTs) in its coverage region. All BSs in the network are connected to each other with a wired backbone network. When a UT moves from one cell to another, the serving BS hands off its responsibilities to the BS in the new cell.

As is characteristic of a terrestrial wireless channel, UTs that are located far from the BS are likely to receive highly attenuated signals. This phenomenon is called path loss. In addition to path loss, UTs that are located close to the periphery of the cell may suffer from inter-cell interference (ICI), i.e., the interference caused by transmissions from the BSs in other cells. Both path loss and ICI reduce the signalto-interference-plus-noise ratio (SINR), and in turn, the achievable data rates, of the UTs, especially those at the cell edge. Hence, there is a need to develop efficient and cost-effective techniques to combat these phenomena in order for future cellular 
networks to satisfy the anticipated consumer demands.

\subsection{Distributed Antenna Systems}

An effective approach to counteract distance-based signal attenuation is to bring the cellular network closer to the UTs. A distributed antenna system (DAS) [1] is a promising candidate architecture to attain this goal. In a DAS, multiple antenna ports are dispersed throughout a cell, and the BS in the cell is connected to these ports with high-speed communication links, such as optical fiber. Using such a configuration, the DAS helps enable more ubiquitous high data-rate coverage throughout the cell.

Although a DAS reduces the performance-degrading effects of path loss, particularly for cell-edge UTs, this architecture does not inherently mitigate ICI. In other words, despite the fact that the desired signal strength at a cell-edge UT increases due to the reduced access distance to the serving BS, the ICI is also stronger as a result of the reduced distance to some of the ports in other cells. Hence, there remains a need to implement appropriate processing techniques that mitigate ICI. One such set of techniques, known as coordinated multi-point (CoMP) transmission and reception, is described in the next section.

\subsection{Coordinated Multi-Point Transmission and Reception}

The conventional approach for mitigating ICI is a sparse reuse of frequency-time resource blocks (RBs), i.e., by avoiding the assignment of a particular RB to cells that have relatively small geographic separation. Despite the efficacy of this approach, the available spectrum is used inefficiently. Since the spectrum is a limited and expensive resource, the cellular industry has recently begun to implement more aggressive reuse patterns, and seek alternative and more cost-effective ICI mitigation methods for future cellular networks. A promising set of techniques that achieves this objective is 
CoMP, which is also known as multi-cell multiple-input multiple-output (MIMO) or network MIMO.

Under the CoMP framework, a high-speed backbone network that connects the BSs to each other is used to establish coordination between these BSs. By means of this coordination, various ICI mitigation techniques can be implemented, thus increasing the SINR of cell-edge UTs. Although the concept of BS coordination has existed in the research community for a relatively long period of time (see, e.g., $[2$, $3,4]$ ), CoMP has recently been proposed as a candidate technology for enhancing data rates in future cellular networks $[5,6,7]$. In particular, CoMP is envisioned to be an integral part of the 3rd Generation Partnership Project (3GPP) Long Term

Evolution (LTE) Advanced specifications (Release 11 and beyond) [8, 9].

Although coordination among BSs is the most common type of CoMP, it can be observed that transmission from multiple distributed antenna ports in a DAS to a particular UT is also an intra-cell level of CoMP. Hence, the DAS architecture and CoMP schemes (both at the intra-cell and inter-cell levels) complement each other to collectively achieve improved performance in cellular networks. In fact, the use of CoMP schemes in conjunction with cellular DASs is in consideration for LTEAdvanced; see [8, Section 20.1].

\subsection{Thesis Contributions and Organization}

In this thesis, both intra-cell and inter-cell downlink CoMP schemes are incorporated into a cellular DAS architecture. In doing so, not only are the performance-degrading effects of path loss reduced, but ICI is also mitigated. Hence, significantly improved performance is attained in the cellular network.

An approach that is common to all the chapters in the thesis is port selection, wherein a subset of the available distributed antenna ports in a cell or a cluster of cells are chosen for transmission to each UT. Port selection is motivated by the trade-off 
between the benefit to a particular UT that is achieved by using a large number of ports to transmit to this UT, and the performance loss that is suffered by other UTs as a result of the increased levels of interference. It is shown in the thesis that an individual (per-port or per-antenna) power constraint is desirable to fully realize the performance gains of port selection. In particular, it is demonstrated that, when each port transmits at a fixed power level, significant improvement in the performance can be attained using proper port selection.

Throughout the thesis, a variety of metrics are used to assess the performance of the schemes and algorithms that are developed for the cellular DAS. These include the aggregate cell spectral efficiency per RB, the average aggregate spectral efficiency per cell per RB, and the maximum minimum SINR (i.e., the maximum achievable guarantee on the minimum SINR of the UTs). The first two metrics represent overall performance of a particular cell or the network, while the third one can be related to the performance of cell-edge UTs.

The thesis is organized as follows:

- In Chapter 2, a brief literature review is given for DAS and CoMP, in addition to suitable information-theoretic models for cellular systems employing various levels of CoMP. The existing results for these models are used to assess the challenges involved in the design of downlink CoMP schemes for a cellular DAS.

- In Chapter 3, two closely-related precoding schemes are developed for a cellular DAS with a priori port selection and coordinated transmission from multiple distributed antenna ports. These schemes are extensions of existing ones for a co-located antenna system (CAS), i.e., a conventional cellular system. The performance gains of the DAS schemes over the corresponding CAS schemes are demonstrated using simulation. The results in this chapter provide important insights, which are used to develop the algorithms described in the next chapter. 
- Chapter 4 contains the main contribution of thesis. In this chapter, a two-stage approach is proposed for determining an approximate set of binary port states and the corresponding beam steering coefficients that collectively maximize the minimum SINR in a cellular DAS with inter-cell coordination and fixed transmit power levels at each port. In each stage of this approach, the semidefinite relaxation (SDR) technique with Gaussian randomization is used to efficiently generate a close-to-optimal solution to an optimization problem that is nondeterministic polynomial-time hard (NP-hard). Hence, the proposed approach is capable of providing an approximate solution to the original NP-hard problem in polynomial time, and simulation is used to demonstrate its efficacy.

- The thesis is concluded in Chapter 5 with a summary of the main contributions and a discussion of potential ideas for future work in the area of downlink CoMP transmission in a cellular DAS.

\subsection{Publications}

\section{Chapter 3}

- Talha Ahmad, Saad Al-Ahmadi, Halim Yanikomeroglu, and Gary Boudreau, "Downlink linear transmission schemes in a single-cell distributed antenna system with port selection," in Proceedings of IEEE Vehicular Technology Conference (VTC2011-Spring), May 2011.

\section{Chapter 4}

- Talha Ahmad, Ramy Gohary, Halim Yanikomeroglu, Saad Al-Ahmadi, and Gary Boudreau, "Coordinated port selection and beam steering optimization in a multi-cell distributed antenna system using semidefinite relaxation," under review in IEEE Transactions on Wireless Communications. 
- Talha Ahmad, Ramy Gohary, Halim Yanikomeroglu, Saad Al-Ahmadi, and Gary Boudreau, "Coordinated max-min fair port selection in a multi-cell distributed antenna system using semidefinite relaxation," submitted to IEEE Global Communications Conference (Globecom 2011) Workshop on Distributed Antenna Systems for Broadband Mobile Communications, December 2011. 


\section{Chapter 2}

\section{Background}

\subsection{Related Works on Distributed Antenna Systems}

Distributed antenna systems were originally introduced in [1] to fill coverage gaps in indoor wireless networks, and early research on DASs was in the context of such networks (see, e.g., [10]).

Early works on the integration of DASs in cellular networks appeared in [11]-[14], and these were primarily focussed on code division multiple access (CDMA) based systems. In addition to incorporating this architecture into cellular networks, it was shown in these works that dispersing the antennas of the BS over the geographic area of the cell results in increased capacity as well as reduced transmit power levels.

In [15], the uplink outage signal-to-noise ratio (SNR) performance of a generalized MIMO DAS with multi-antenna ports and multi-antenna UTs was evaluated for various diversity combining schemes using a composite fading channel model. This work was followed by [16], wherein the uplink and downlink outage capacity achieved by such a generalized MIMO DAS was investigated. Furthermore, in [17], it was demonstrated that a DAS achieves significantly higher capacity as compared to a traditional MIMO system, i.e., a CAS.

Although the performance improvements offered by a DAS as compared to a CAS are clear, the literature regarding transmission and power allocations schemes in a DAS was relatively limited in the past. Recently, however, novel DAS signal 
processing and resource allocation schemes, particularly those involving coordination between multiple ports and/or cells, have begun to appear; see, e.g., $[18,19]$ (both of these works will be discussed in further detail in Section 3.1).

A comprehensive overview of the DAS architecture and related coordinated transmission and power allocation schemes, as well as other resource allocation schemes, can be found in $[20,21]$.

\subsection{Related Works on CoMP}

The term coordinated multi-point transmission and reception serves as an umbrella for techniques and schemes that utilize coordination between multiple transmitters and/or receivers to mitigate interference. In the context of cellular networks, CoMP generally refers to coordination between BSs for both uplink and downlink. The focus in this thesis is on the latter scenario, and hence, an overview of literature related to the downlink case will be provided in this section.

The set of techniques that fall under the CoMP umbrella spans both the physical and medium access control (MAC) layers. For instance, physical layer CoMP techniques include coordinated multi-cell beamforming and precoding (see, e.g., [22, 23]), while MAC layer techniques include coordinated UT scheduling and power allocation (see, e.g., [24]). The focus herein is on the earlier set of techniques.

An assumption that is commonly made when designing CoMP schemes is that all the BSs have perfect channel state information (CSI) for all the UTs in the coordination region. Furthermore, it is generally assumed that the coordination between the BSs is perfect, i.e., the backbone links have negligible delay, are relatively error-free, and have infinite capacity. Although such assumptions simplify the design, they are not necessarily applicable in practice, especially when the number of coordinating BSs is large. To alleviate such impractical assumptions while maintaining design tractability, clustered coordination schemes have been proposed $[25,26]$. In such 
schemes, a large cellular network is divided into smaller clusters of cells. The BSs of the cells in a particular cluster coordinate their transmissions, but there is limited or no inter-cluster coordination. Additionally, in some works, such as [27] and [28], the heavy overhead on the backbone is reduced by designing schemes that rely only on locally available CSI.

Although CoMP techniques impose a heavier load on the backbone and also have relatively strict delay and error threshold requirements, their potential advantages in terms of the achievable data rates have been demonstrated both numerically [29] and analytically [30]. They have also been shown to outperform conventional noncoordinating cellular networks, even in the presence of moderate amounts of channel estimation error [31]. Furthermore, in [32], a performance trade-off has been investigated between BS coordination and denser BS deployment in future cellular networks.

A detailed overview of existing CoMP schemes, their performance, and the challenges involved in their implementation, can be found in [33] and [34].

\subsection{Information-Theoretic Background}

In this section, the underlying information-theoretic models will be presented for cellular systems with different levels of coordination.

Consider a single-cell system in which a multi-antenna BS transmits to one or more $\mathrm{UTs}^{1}$. If the number of UTs in the cell is exactly one, then such a system can be modelled as a point-to-point MIMO channel, for which, the capacity can be achieved using the singular value decomposition (SVD) based water-filling scheme proposed in [35]. However, if there are multiple UTs in the cell, then such a system can be modelled as a Gaussian MIMO broadcast channel (BC), for which, the dirty paper coding (DPC) scheme [36] is known to achieve the capacity [37]. However, DPC is difficult to implement in practice, and hence, there exist various linear and

\footnotetext{
${ }^{1}$ The antennas of the BS may be either co-located or distributed.
} 
non-linear sub-optimal schemes that are more practical (see, e.g., [38]-[44]). Such schemes will be the focus of the discussion in Chapter 3.

Now, let us consider a more general multi-cell system in which the BS in each cell operates independently as described above. Such a system can be treated as a MIMO interference channel (IC). Although information-theoretic results exist for some special cases (see, e.g., [45]), the capacity region of a general MIMO IC has not yet been characterized. It is, therefore, difficult to gauge the performance of transmission schemes designed for such systems. A simplifying strategy is to establish full multi-cell coordination, which results in a larger MIMO BC. This is the approach taken to model the system in Chapter 4. 


\section{Chapter 3}

\section{Coordinated Multi-Point Downlink Transmission Schemes}

In this chapter, the focus is on the design and performance evaluation of downlink transmission schemes in a cellular DAS, wherein a subset of the available multiantenna ports of a cell transmit in a coordinated manner to serve one or more multiantenna UTs in a particular RB.

\subsection{Related Literature}

As mentioned in Section 2.3, a single-cell CAS with multi-antenna UTs can be modeled as a Gaussian MIMO BC, for which the optimal transmission scheme is DPC. However, DPC is difficult to implement in practice due to its computational complexity, and several sub-optimal transmission schemes have been proposed for mitigating inter-user interference. In [38], the zero-forcing dirty paper coding (ZF-DPC) scheme is proposed, which uses LQ decomposition on the aggregate channel matrix, which consists of the channel matrices of all single-antenna UTs, to eliminate a part of the inter-user interference. The remaining interference is mitigated by means of successive dirty paper encoding. In [38], ZF-DPC is shown to be asymptotically optimal with increasing SNR. In [40]-[43], this scheme is extended to incorporate multi-antenna UTs. A linear scheme that mitigates inter-user interference is zero-forcing beamforming (ZFBF), which spatially orthogonalizes all single-antenna UTs by using the 
pseudo-inverse of the aggregate channel matrix as the precoding matrix. An extension of this scheme for multi-antenna UTs is block diagonalization (BD) [39], in which the precoding matrix of each UT is designed such that its transmitted signal is in the null space of the channel matrices of the other UTs.

With the incorporation of port selection, the CAS-based versions of the schemes described above cannot be directly applied to a DAS, and processing modifications are necessary. In this chapter, the ZF-DPC and BD schemes are extended to fit the cellular DAS architecture.

In [18], the performance of various multi-user transmission schemes, including BD, is explored in the context of a single-cell DAS with multi-antenna ports. However, the schemes presented in [18] do not incorporate port selection. Furthermore, singleantenna UTs are mainly assumed and spatial multiplexing of multiple data streams for each UT is not included. Both these features are included in the schemes described herein. In [19], port selection is explored for a multi-user cellular DAS. However, the UTs are orthogonalized through orthogonal frequency division multiplexing (OFDM). In contrast, orthogonalization is achieved using spatial precoding in this chapter.

\subsection{Background: Basic Linear Algebra}

In this section, a brief overview will be provided for matrix analysis topics that are relevant to the formulation in this chapter. Before proceeding, however, it is necessary to state that throughout this chapter and the rest of the thesis, scalars are denoted by lower-case regular-face letters, vectors are denoted by lower-case bold-face letters, and matrices are denoted by upper-case bold-face letters. 


\subsubsection{Eigenvectors and Eigenvalues}

Let $\boldsymbol{A}$ be a square matrix. A non-zero vector $\boldsymbol{v}$ is called a right eigenvector of $\boldsymbol{A}$ if there exists a scalar, $\lambda$, such that

$$
\boldsymbol{A v}=\lambda \boldsymbol{v}
$$

and it is called a left eigenvector of $\boldsymbol{A}$ if there exists a scalar, $\lambda$, such that

$$
\boldsymbol{v}^{H} \boldsymbol{A}=\lambda \boldsymbol{v}^{H}
$$

In (3.1) and (3.2), $\lambda$ is called an eigenvalue of $\boldsymbol{A}$ corresponding to $\boldsymbol{v}$ and $\boldsymbol{v}^{H}$, respectively [46, Section 6.1].

\subsubsection{Null Space of a Matrix}

Let $\boldsymbol{B} \in \mathbb{C}^{m \times n}$ be a rectangular matrix. The null space, which is also referred to as the kernel, of the matrix $\boldsymbol{B}$ is the set of vectors $\boldsymbol{y}$ such that

$$
B y=0 .
$$

The dimension of the null space of a matrix is called the nullity of this matrix [46, Section 4.5].

\subsubsection{Rank of a Matrix}

The rank of a matrix is defined as the minimum number of linearly independent columns or rows of this matrix [46, Section 4.5]. This value is related to the nullity of the matrix as follows. Consider the matrix $\boldsymbol{B}$ defined above, and let $r$ denote the rank of $\boldsymbol{B}$. Then, the nullity of $\boldsymbol{B}$ is equal to $n-r$. 


\subsubsection{Singular Value Decomposition}

Consider the rectangular matrix $\boldsymbol{B}$ defined in Section 3.2.2. Any such matrix can be factorized as follows:

$$
\boldsymbol{B}=\boldsymbol{U} \boldsymbol{\Sigma} \boldsymbol{V}^{H}
$$

where $\boldsymbol{U} \in \mathbb{C}^{m \times m}$ and $\boldsymbol{V} \in \mathbb{C}^{n \times n}$ are unitary matrices ${ }^{1}$ and $\boldsymbol{\Sigma} \in \mathbb{R}^{m \times n}$ is a diagonal matrix of the form

$$
\boldsymbol{\Sigma}=\left[\begin{array}{lll}
\sigma_{1} & & \\
& \ddots & \\
& & \sigma_{p}
\end{array}\right]
$$

where $p=\min (m, n)$, and $\sigma_{1}, \ldots, \sigma_{p}$ are called the singular values of $\boldsymbol{B}$ [46, Section 7.1]. These singular values are generally arranged in decreasing order, and are related to the eigenvalues as follows:

$$
\lambda_{i}=\sigma_{i}^{2}, \quad i=1, \ldots, p
$$

\subsection{Single-Cell Processing}

In this section, the DAS BD and DAS ZF-DPC schemes will be developed under the assumption that the BS in each cell operates independently from those in other cells.

\subsubsection{System Model}

Consider a cellular DAS consisting of $M$ cells which use the same set of frequencytime RBs each. The BS in each cell is connected to $L$ distributed $N_{t}$-antenna ports with high-speed communication links (e.g., optical fiber). Additionally, the BS in each cell has reliable knowledge of the gains between the ports and each UT in this

\footnotetext{
${ }^{1}$ A matrix $\boldsymbol{U}$ is said to be unitary if $\boldsymbol{U}^{H} \boldsymbol{U}=\boldsymbol{U} \boldsymbol{U}^{H}=\boldsymbol{I}$, where $\boldsymbol{I}$ is an identity matrix [46, Section 5.3.1].
} 
cell. A multi-user system that uses a narrow-band multiple access scheme, such as an orthogonal frequency division multiple access (OFDMA) based one, is considered. In this system, there are $K$ UTs per RB in each cell, and these UTs are equipped with $N_{r}$ antennas each.

Let $\mathcal{S}_{m}$ represent the set of indices of all ports in the $m$-th cell, where $\left|\mathcal{S}_{m}\right|=L$ and $|\cdot|$ denotes the cardinality of the set argument. Let $\mathcal{A}_{m}$ represent the set of active ports in the $m$-th cell $\left(\mathcal{A}_{m} \subseteq \mathcal{S}_{m}\right)$. Also, let $\mathcal{C}_{k m}$ denote the set of ports in the $m$-th cell that transmit to the $k$-th UT in this cell in a coordinated manner, and let $\mathcal{I}_{k m}$ denote the set of ports in the $m$-th cell that cause interference to this UT. Hence, $\mathcal{C}_{k m} \bigcup \mathcal{I}_{k m}=\mathcal{A}_{m}$ for all $k, m$, and $\bigcup_{k=1}^{K} \mathcal{C}_{k m}=\bigcup_{k=1}^{K} \mathcal{I}_{k m}=\mathcal{A}_{m}$ for all $m$.

Before proceeding with a description of the signal and channel models, it is emphasized that all formulation that follows is applicable to a single RB. However, explicit reference to a particular RB index is omitted for notational brevity.

In Fig. 3.1, a single cell of the cellular DAS with $L=7$ and $K=3$ is shown, and the signal model that follows in this section is illustrated.

\section{Signal Model}

The received signal, $\boldsymbol{y}_{k m} \in \mathbb{C}^{N_{r}}$, of the $k$-th UT in the $m$-th cell can be expressed as

$$
\boldsymbol{y}_{k m}=\boldsymbol{H}_{m k m} \boldsymbol{x}_{m}+\sum_{n=1, n \neq m}^{M} \boldsymbol{H}_{n k m} \boldsymbol{x}_{n}+\boldsymbol{n}_{k m}, \quad k=1, \ldots, K, m=1, \ldots, M
$$

where, $\boldsymbol{H}_{n k m} \in \mathbb{C}^{N_{r} \times\left|\mathcal{A}_{n}\right| N_{t}}$ is a matrix consisting of the complex-valued channel gains between all active ports in the $n$-th cell and the $k$-th UT in the $m$-th cell, $\boldsymbol{n}_{k m} \in \mathbb{C}^{N_{T}}$ is a zero-mean complex Gaussian noise vector with covariance matrix $E\left\{\boldsymbol{n}_{k m} \boldsymbol{n}_{k m}^{H}\right\}=$ $\sigma^{2} \boldsymbol{I}_{N_{r}}$, where $\boldsymbol{I}_{N_{r}}$ denotes an $N_{r} \times N_{r}$ identity matrix, and $\boldsymbol{x}_{m} \in \mathbb{C}^{\left|\mathcal{A}_{m}\right| N_{t}}$ is the signal 

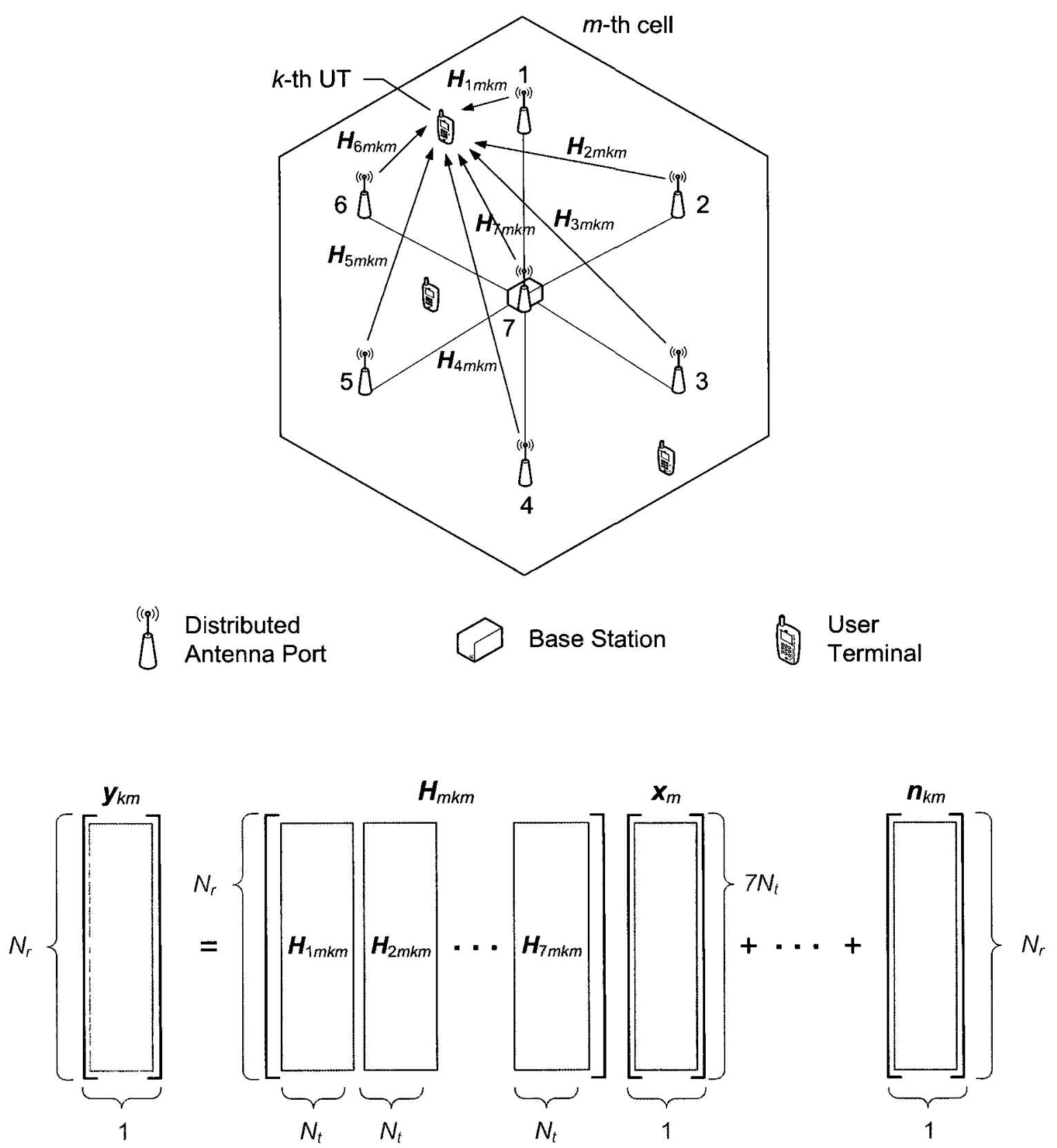

Figure 3.1: A single DAS cell with $K=3$ UTs per RB and $L=7$ ports, and an illustration of the general signal model. In this example, without loss of generality, $\mathcal{A}_{m}=\mathcal{S}_{m}=\{1,2, \ldots, 7\}$. 
transmitted from the active ports in the $m$-th cell. This signal is of the form

$$
\boldsymbol{x}_{m}=\sum_{k=1}^{K} \boldsymbol{F}_{k m}^{\prime} \boldsymbol{u}_{k m}
$$

where $\boldsymbol{u}_{k m} \in \mathbb{C}^{N_{r}}$ denotes the data vector of the $k$-th UT in the $m$-th cell, and $\boldsymbol{F}_{k m}^{\prime} \in \mathbb{C}^{\left|\mathcal{A}_{m}\right| N_{t} \times N_{r}}$ is its precoding matrix. For convenience, this precoding matrix will be designed in two separate stages: beamforming and power allocation. To facilitate this two-stage approach, it is chosen to be of the form $\boldsymbol{F}_{k m}^{\prime}=\boldsymbol{F}_{k m} \boldsymbol{\Lambda}_{k m}^{\frac{1}{2}}$, where $\boldsymbol{F}_{k m} \in \mathbb{C}^{\left|\mathcal{A}_{m}\right| N_{t} \times N_{r}}$ is the transmit beamforming matrix and $\boldsymbol{\Lambda}_{k m} \in \mathbb{C}^{N_{r} \times N_{r}}$ is a diagonal power allocation matrix. Using this notation, (3.6) can be re-written as

$$
\begin{aligned}
\boldsymbol{y}_{k m}=\boldsymbol{H}_{m k m} \boldsymbol{F}_{k m} \boldsymbol{\Lambda}_{k m}^{\frac{1}{2}} \boldsymbol{u}_{k m}+\boldsymbol{H}_{m k m} & \sum_{\jmath=1, \jmath \neq k}^{K} \boldsymbol{F}_{\jmath m} \boldsymbol{\Lambda}_{\jmath m}^{\frac{1}{2}} \boldsymbol{u}_{\jmath m}+ \\
& \sum_{n=1, n \neq m}^{M} \boldsymbol{H}_{n k m} \sum_{\jmath=1}^{K} \boldsymbol{F}_{\jmath n} \boldsymbol{\Lambda}_{\jmath n}^{\frac{1}{2}} \boldsymbol{u}_{\jmath n}+\boldsymbol{n}_{k m},
\end{aligned}
$$

where the second and third terms on the right-hand side of (3.8) represent the intracell and inter-cell interference experienced by the $k$-th UT in the $m$-th cell, respectively. In Sections 3.3.2 and 3.3.3, the focus will on be the design of the precoding matrix to mitigate intra-cell interference. The ICI will be treated as additional noise.

The ports in each cell in the cellular DAS considered herein are subject to a total power constraint, $P_{t}$; that is, $E\left\{\boldsymbol{x}_{m} \boldsymbol{x}_{m}^{H}\right\} \leq P_{t}$ for all $m$, where $E\{\cdot\}$ denotes the expectation operation. Assuming that the data vectors, $\boldsymbol{u}_{k m}$, are zero-mean with identity covariance matrix for all $k, m$, this constraint can be expressed as

$$
\sum_{k=1}^{K} \operatorname{Tr}\left(\boldsymbol{S}_{k m}\right) \leq P_{t}, \quad m=1, \ldots, M
$$

where $\boldsymbol{S}_{k m}=\boldsymbol{F}_{k m}^{\prime} \boldsymbol{F}_{k m}^{\prime H}$ is the transmit covariance matrix of the $k$-th UT in the $m$-th cell, and $\operatorname{Tr}(\cdot)$ is the trace operator. 
It is important to note that a per-port or per-antenna power constraint would be more practical for a cellular DAS since the ports are geographically dispersed throughout the cell, and each antenna is equipped with a separate power amplifier. Furthermore, it will be shown later that the performance gains promised by port selection are not fully realized in a system that is subject to a total power constraint. However, the total power constraint is considered herein due to the following two reasons. Firstly, it enables a fair comparison between the performance of the DAS transmission schemes presented herein and that of corresponding schemes in a CAS, which is generally subject to a total power constraint. Secondly, the design of transmission schemes is significantly more challenging if the system is subject to an individual power constraint. Although there exist numerical algorithms that use convex optimization techniques to attain this goal (see, e.g., [47]-[49]), no closedform analytically derivable optimal precoding scheme satisfying an individual power constraint has yet been developed.

If a per-port power constraint was to be imposed upon the system, the inequality in (3.9) would be revised to

$$
\sum_{k=1}^{K} \operatorname{Tr}\left(\boldsymbol{S}_{\jmath k m}\right) \leq P_{\jmath}, \quad \forall j \in \mathcal{A}_{m}
$$

where $P_{\jmath}$ is the power constraint of the $j$-th port of the $m$-th cell, $\boldsymbol{S}_{\jmath k m}=\boldsymbol{F}_{j k m}^{\prime} \boldsymbol{F}_{\jmath k m}^{\prime H}$ is the transmit covariance matrix for the signal transmitted by the $j$-th port in the $m$-th cell to the $k$-th UT in this cell, such that $\boldsymbol{F}_{k m}^{\prime}=\left[\boldsymbol{F}_{1 k m}^{\prime H} \ldots \boldsymbol{F}_{\left|\mathcal{A}_{m}\right| k m}^{\prime H}\right]^{H}[47]$.

\section{Channel Model}

The matrix $\boldsymbol{H}_{n k m}$ in the DAS signal model above represents a quasi-static frequencyflat wireless channel with Rayleigh fading, log-normal shadowing, and path loss com- 
ponents. This matrix can be expressed as

$$
\boldsymbol{H}_{n k m}=\left[\begin{array}{llll}
\boldsymbol{H}_{1 n k m} & \boldsymbol{H}_{2 n k m} & \ldots & \boldsymbol{H}_{\left|\mathcal{A}_{n}\right| n k m}
\end{array}\right], \quad k=1, \ldots, K, m, n=1, \ldots, M,
$$

where

$$
\boldsymbol{H}_{\jmath n k m}=\sqrt{\rho\left(d_{j n k m}\right) s_{\jmath n k m}} \boldsymbol{H}_{j n k m}^{\prime} .
$$

In (3.12), $d_{\jmath n k m}$ is the distance between the $j$-th port in the $n$-th cell and the $k$-th UT in the $m$-th cell, and $\rho(\cdot)$ is a path loss function, which depends on the propagation environment. Shadowing is represented by $s_{j n k m}$, which is log-normal distributed with $0 \mathrm{~dB}$ mean and standard deviation $\sigma_{s}$ in $\mathrm{dB}$. Multipath fading is represented by the matrix $\boldsymbol{H}_{\text {Jnkm }}^{\prime} \in \mathbb{C}^{N_{r} \times N_{t}}$. Each element of this matrix is complex Gaussian distributed with zero mean and unit variance.

The corresponding channel matrix for the CAS can be written as

$$
\boldsymbol{H}_{n k m}=\sqrt{\rho\left(d_{\mathrm{BS}_{n}, k m}\right) s_{\mathrm{BS}_{n}, k m}} \boldsymbol{H}_{n k m}^{\prime}
$$

where $s_{\mathrm{BS}_{n}, k m}$ denotes log-normal shadowing between $\left|\mathcal{A}_{n}\right| N_{t}$ co-located antennas at the BS of the $n$-th cell and the $k$-th UT in the $m$-th cell, and it has the same statistics as $s_{j n k m}$ above. The distance between this BS and this UT is denoted by $d_{\mathrm{BS}_{n}, k m}$, and $\boldsymbol{H}_{n k m}^{\prime} \in \mathbb{C}^{N_{r} \times\left|\mathcal{A}_{n}\right| N_{t}}$ is the multipath fading coefficient matrix.

\subsubsection{DAS Block Diagonalization}

Considering the first term on the right-hand side of (3.8), the rows of $\boldsymbol{F}_{k m}$ corresponding to those columns of $\boldsymbol{H}_{m k m}$ that represent the transmit antennas of the ports in $\mathcal{I}_{k m}$, can be set to zero, since those particular ports do not transmit desired signals to the $k$-th UT in the $m$-th cell. The remaining non-zero rows of $\boldsymbol{F}_{k m}$ can then constitute the submatrix $\hat{\boldsymbol{F}}_{k m} \in \mathbb{C}^{\left|\mathcal{C}_{k m}\right| N_{t} \times N_{r}}$. This reduction is performed to 
simplify the beamforming matrix design.

Eliminating intra-cell interference requires the following condition (which is commonly referred to as the zero-forcing condition in the literature) to be satisfied: $\boldsymbol{H}_{m \jmath m} \boldsymbol{F}_{k m}=0, \forall j \neq k$. Using the dimensional reduction described above, this condition can be expressed as

$$
\hat{\boldsymbol{H}}_{m \jmath m} \hat{\boldsymbol{F}}_{k m}=0, \forall j \neq k
$$

where $\hat{\boldsymbol{H}}_{m k m} \in \mathbb{C}^{N_{r} \times\left|\mathcal{C}_{k m}\right| N_{t}}$ is a channel matrix containing only those columns of $\boldsymbol{H}_{m k m}$ that correspond to the transmit antennas of the ports in $\mathcal{C}_{k m}$. To satisfy this zero-forcing constraint, a matrix known as the interference matrix can be defined for each UT. This matrix is a collection of the complex-valued channel gains between the antennas of the ports that are selected for transmission to the a particular UT in the $m$-th cell and the antennas of the other $K-1$ UTs in this cell. In particular, the interference matrix of the $k$-th UT in the $m$-th cell is defined as

$$
\tilde{\boldsymbol{H}}_{k m} \triangleq\left[\begin{array}{llllll}
\overline{\boldsymbol{H}}_{m 1 m}^{\left(\mathcal{C}_{k m}\right) H} & \ldots & \overline{\boldsymbol{H}}_{m(k-1) m}^{\left(\mathcal{C}_{k m}\right) H} & \overline{\boldsymbol{H}}_{m(k+1) m}^{\left(\mathcal{C}_{k m}\right) H} & \ldots & \overline{\boldsymbol{H}}_{m K m}^{\left(\mathcal{C}_{k m}\right) H}
\end{array}\right]^{H}, \quad k=1, \ldots, K
$$

where $\overline{\boldsymbol{H}}_{m \jmath m}^{\left(\mathcal{C}_{k m}\right)} \in \mathbb{C}^{N_{r} \times\left|\mathcal{C}_{k m}\right| N_{t}}$ is a submatrix containing only those columns of $\boldsymbol{H}_{m \jmath m}$ that correspond to the transmit antennas of the ports in $\mathcal{C}_{k m}$. The goal is to design the beamforming matrix of the $k$-th UT such that it is orthogonal to the interference matrix corresponding to this UT.

Let $\tilde{L}_{k m} \triangleq \operatorname{rank}\left(\tilde{\boldsymbol{H}}_{k m}\right)$, where $\operatorname{rank}(\cdot)$ denotes the rank of the matrix argument. Assuming that $\tilde{\boldsymbol{H}}_{k m}$ is full-rank for all $k, m, \tilde{L}_{k m}=\min \left\{N_{r}(K-1),\left|\mathcal{C}_{k m}\right| N_{t}\right\}^{2}$. To satisfy the zero-forcing constraint in (3.14), the columns of the beamforming matrix, $\hat{\boldsymbol{F}}_{k m}$, must span the null space of $\tilde{\boldsymbol{H}}_{k m}$. To obtain candidate vectors for the columns

\footnotetext{
${ }^{2}$ This follows from the reasonable assumption that the UTs are located sufficiently far apart such that their fading coefficients are independent [50].
} 
of $\hat{\boldsymbol{F}}_{k m}$, the following SVD is performed.

$$
\tilde{\boldsymbol{H}}_{k m}=\tilde{\boldsymbol{U}}_{k m} \tilde{\boldsymbol{\Sigma}}_{k m}\left[\tilde{\boldsymbol{V}}_{k m}^{(1)} \tilde{\boldsymbol{V}}_{k m}^{(0)}\right]^{H}
$$

where $\tilde{\boldsymbol{V}}_{k m}^{(1)}$ contains in its columns the first $\tilde{L}_{k m}$ right singular vectors of $\tilde{\boldsymbol{H}}_{k m}$, and $\tilde{\boldsymbol{V}}_{k m}^{(0)}$ contains the remaining $\left|\mathcal{C}_{k m}\right| N_{t}-\tilde{L}_{k m}$ right singular vectors. The columns of $\tilde{\boldsymbol{V}}_{k m}^{(0)}$ form an orthogonal basis for the null space of $\tilde{\boldsymbol{H}}_{k m}$, and hence, a linear combination of these columns can be used to design $\hat{\boldsymbol{F}}_{k m}$. It can be noted that such a design is only possible if there is a sufficient number of columns in $\tilde{\boldsymbol{V}}_{k m}^{(0)}$. Hence, to satisfy the zero-forcing constraint, it is necessary that $\tilde{\boldsymbol{V}}_{k m}^{(0)}$ is non-empty for all $k, m$. This constraint can equivalently be expressed as

$$
N_{r}(K-1)<\min _{k=1,, K}\left(\left|\mathcal{C}_{k m}\right| N_{t}\right), \quad m=1, \ldots, M
$$

This is the dimensionality constraint of a cellular DAS with port selection and singlecell processing, which limits the number of UTs that can be simultaneously served in a particular RB such that the zero-forcing condition in (3.14) is satisfied ${ }^{3}$.

Now, assuming that $\tilde{\boldsymbol{V}}_{k m}^{(0)}$ has a sufficient number of columns, a subset of these vectors can be chosen such that the effective channel gain is maximized. This can be achieved by performing the following SVD [39].

$$
\hat{\boldsymbol{H}}_{m k m} \tilde{\boldsymbol{V}}_{k m}^{(0)}=\hat{\boldsymbol{U}}_{k m}\left[\begin{array}{cc}
\hat{\boldsymbol{\Sigma}}_{k m} & \mathbf{0} \\
\mathbf{0} & \mathbf{0}
\end{array}\right]\left[\hat{\boldsymbol{V}}_{k m}^{(1)} \hat{\boldsymbol{V}}_{k m}^{(0)}\right]^{H}
$$

where $\hat{\boldsymbol{\Sigma}}_{k m} \in \mathbb{C}^{\bar{L}_{k m} \times \bar{L}_{k m}}$ is a diagonal matrix comprised of the non-zero singular values of $\hat{\boldsymbol{H}}_{m k m} \tilde{\boldsymbol{V}}_{k m}^{(0)}$ as its diagonal elements, $\bar{L}_{k m}=\operatorname{rank}\left(\hat{\boldsymbol{H}}_{m k m} \tilde{\boldsymbol{V}}_{k m}^{(0)}\right)$, and $\hat{\boldsymbol{V}}_{k m}^{(1)}$ contains

\footnotetext{
${ }^{3}$ The DAS dimensionality constraint does not, however, impose a strict limit on the overall number of active UTs since a different set of UTs can be selected for service in another RB using an appropriate scheduling algorithm.
} 
the first $N_{r}$ right singular vectors of $\hat{\boldsymbol{H}}_{m k m} \tilde{\boldsymbol{V}}_{k m}^{(0)}$. Then, $\hat{\boldsymbol{F}}_{k m}$ can be expressed as

$$
\hat{\boldsymbol{F}}_{k m}=\tilde{\boldsymbol{V}}_{k m}^{(0)} \hat{\boldsymbol{V}}_{k m}^{(1)}
$$

It is noted that this transmit beamforming submatrix is designed to only mitigate inter-user interference in the $m$-th cell. Although completely orthogonalizing all the data streams at the BS would reduce the processing complexity at the UTs, this approach has been shown in [39] to be sub-optimal in terms of the maximum achievable aggregate spectral efficiency. Therefore, each UT is assigned the task of separating its corresponding data streams. This can be done by using the decoding matrix $\hat{\boldsymbol{U}}_{k m}^{H}$, where $\hat{\boldsymbol{U}}_{k m}$ is obtained from (3.18).

After designing the beamforming matrix, the next step is to allocate the total transmit power $P_{t}$ to the multiple data streams, such that the aggregate cell spectral efficiency is maximized. This can be achieved using the water-filling technique [51].

Let $\hat{\boldsymbol{\Sigma}}_{m}$ be a diagonal matrix that contains in its main diagonal the singular values corresponding to each UT in the $m$-th cell, i.e.,

$$
\hat{\mathbf{\Sigma}}_{m}=\ominus_{k=1}^{K} \hat{\boldsymbol{\Sigma}}_{k m}, \quad m=1, \ldots, M
$$

where $\oplus$ denotes the direct sum operation $[52 \text {, Section } 0.9 .2]^{4}$. The optimal power allocation matrix, $\boldsymbol{\Lambda}_{m}$, which is of the form

$$
\boldsymbol{\Lambda}_{m}=\oplus_{k=1}^{K} \boldsymbol{\Lambda}_{k m}, \quad m=1, \ldots, M
$$

can then be obtained by performing water-filling on the diagonal elements of $\boldsymbol{\Sigma}_{m}$.

Using the beamforming, power allocation, and decoding matrices described above, the aggregate cell spectral efficiency of the $m$-th cell in a particular RB can be ex-

\footnotetext{
${ }^{4}$ The direct sum of matrices is notationally equivalent to forming a block diagonal matrix with the argument matrices constituting its block diagonal entries.
} 
pressed as

$$
R_{m, \mathrm{BD}}=\sum_{k=1}^{K} \log _{2} \frac{\left|\boldsymbol{\Theta}+\left(\hat{\boldsymbol{U}}_{k m}^{H} \boldsymbol{H}_{m k m} \boldsymbol{F}_{k m} \boldsymbol{\Lambda}_{k m}^{\frac{1}{2}}\right)^{2}\right|}{|\boldsymbol{\Theta}|}
$$

where $|\cdot|$ denotes the determinant of the matrix argument, and

$$
\boldsymbol{\Theta}=\sigma^{2} \boldsymbol{I}_{N_{r}}+\sum_{n=1, n \neq m}^{M} \sum_{\jmath=1}^{K}\left(\hat{\boldsymbol{U}}_{k m}^{H} \boldsymbol{H}_{n k m} \boldsymbol{F}_{j n} \boldsymbol{\Lambda}_{\jmath n}^{\frac{1}{2}}\right)^{2}
$$

It is again emphasized that the total power constraint limits the performance improvements promised by port selection in a multi-cell DAS. Additionally, by its very nature, the water-filling algorithm may effectively perform port selection by limiting or eliminating the transmit power of certain ports. However, the total power constraint is considered here for tractability reasons. If the system was subject to an individual (per-port or per-antenna) power constraint, the power allocation stage (involving water-filling) described above would be appropriately replaced.

\subsubsection{DAS Zero-Forcing Dirty Paper Coding}

In this section, the ZF-DPC scheme, and in particular, the one described in [41] for multi-antenna UTs, is extended to the DAS with port selection.

Let $\pi$ denote a permutation operator for UT ordering. In the ZF-DPC scheme, successive dirty paper encoding is used to generate the data vector of the $\pi(k)$-th $\mathrm{UT}, \boldsymbol{u}_{\pi(k)}$, such that the interference caused by transmissions intended for the UTs indexed between $\pi(1)$ and $\pi(k-1)$ is eliminated. The remaining intra-cell interference is mitigated using a BD-based zero-forcing technique. In this case, the zero-forcing condition can be written as

$$
\hat{\boldsymbol{H}}_{m \pi(\jmath) m} \hat{\boldsymbol{F}}_{\pi(k) m}=0, \forall k>j .
$$

Similar to the DAS BD scheme in Section 3.3.2, an interference matrix corre- 
sponding to the $\pi(k)$-th UT can be defined as

$$
\tilde{\boldsymbol{H}}_{\pi(k) m} \triangleq\left[\begin{array}{llll}
\overline{\boldsymbol{H}}_{m \pi(1) m}^{\left(\mathcal{C}_{\pi(k) m}\right) H} \overline{\boldsymbol{H}}_{m \pi(2) m}^{\left(\mathcal{C}_{\pi(k) m}\right) H} & \ldots & \overline{\boldsymbol{H}}_{m \pi(k-1) m}^{\left(\mathcal{C}_{\pi(k) m}\right) H}
\end{array}\right]^{H}, \quad k=2, \ldots, K
$$

From this point onward, the design of the transmit beamforming and power allocation matrices is analogous to that described for the DAS BD scheme. In particular, the technique involving two SVD operations (see Section 3.3.2) is used to obtain the precoding and decoding matrices, $\boldsymbol{F}_{\pi(k) m}$ and $\hat{U}_{\pi(k) m}^{H}$, respectively, for each UT.

It can be noted that there is no interference matrix for the $\pi(1)$-th UT because the data vectors of the other $K-1$ UTs are designed to mitigate interference to this UT. In this case, an SVD analogous to the one in (3.18) is applied directly to $\hat{\boldsymbol{H}}_{m \pi(1) m}$, and the resulting singular value matrix, $\hat{\boldsymbol{\Sigma}}_{\pi(1) m}$, is used for power allocation.

Now, using the precoding and decoding matrices described above, the maximum aggregate cell spectral efficiency per RB of the $m$-th cell achieved by the DAS ZF-DPC scheme can be expressed as

$$
R_{m, \mathrm{ZF}-\mathrm{DPC}}=\max _{\pi} \sum_{k=1}^{K} \log _{2} \frac{\left|\boldsymbol{\Xi}+\left(\hat{\boldsymbol{U}}_{\pi(k) m}^{H} \boldsymbol{H}_{m \pi(k) m} \boldsymbol{F}_{\pi(k) m} \boldsymbol{\Lambda}_{\pi(k) m}^{\frac{1}{2}}\right)^{2}\right|}{|\boldsymbol{\Xi}|}
$$

where

$$
\Xi=\sigma^{2} \boldsymbol{I}_{N_{r}}+\sum_{n=1, n \neq m}^{M} \sum_{j=1}^{K}\left(\hat{\boldsymbol{U}}_{\pi(k) m}^{H} \boldsymbol{H}_{n \pi(k) m} \boldsymbol{F}_{\pi(j) n} \boldsymbol{\Lambda}_{\pi(j) n}^{\frac{1}{2}}\right)^{2}
$$

and the maximization over $\pi$ is due to the fact that the UT ordering affects the achievable spectral efficiency. In particular, in the BD-based zero-forcing technique, the precoding matrices of the UTs are designed to lie in the null space of other UTs' channels, whereas successive dirty paper encoding does not incur any such limitation on the precoder design. Therefore, the $\pi(K)$-th UT is the most restricted in terms of the number of available spatial degrees of freedom, while the $\pi(1)$-th UT is the least restricted. It is for this reason that $\mathrm{ZF}-\mathrm{DPC}$ outperforms $\mathrm{BD}$, as will be shown in 
Section 3.5. Although some heuristic UT ordering strategies have been proposed in the literature (see, e.g., [34]), an arbitrary ordering is considered herein for simplicity.

\subsubsection{An Exemplary Configuration}

In this section, the application of the DAS BD and DAS ZF-DPC schemes described in Sections 3.3.2 and 3.3.3, respectively, will be illustrated by means of an example.

Consider the setup shown in Fig. 3.2, in which $M=1, K=3$, and $\mathcal{S}_{1}=$ $\{1,2, \ldots, 7\}$. To evenly cover the geographic area of the cell, six of the ports are distributed uniformly in the cell at a distance of $\frac{2}{3} r_{c}$ from the BS, where $r_{c}$ is the circumradius of the hexagonal cell, and the seventh port is co-located with the BS at the center of the cell. The location of each UT is fixed as shown in Fig. 3.2. Each UT receives desired signals from the two ports that have the shortest distance to it, i.e., $\mathcal{C}_{11}=\{1,2\}, \mathcal{C}_{21}=\{3,4\}$, and $\mathcal{C}_{31}=\{1,6\}$. In this case, ports 5 and 7 are inactive, and hence, $\mathcal{A}_{1}=\{1,2,3,4,6\}$. The channel matrix for each of the UTs can be expressed as

$$
\boldsymbol{H}_{k 1}=\left[\begin{array}{lllll}
\boldsymbol{H}_{11 k 1} & \boldsymbol{H}_{21 k 1} & \boldsymbol{H}_{31 k 1} & \boldsymbol{H}_{41 k 1} & \boldsymbol{H}_{61 k 1}
\end{array}\right], \quad k=1,2,3,
$$

where $\boldsymbol{H}_{j n k m}$ is as described in (3.12). The channel submatrices $\hat{\boldsymbol{H}}_{1 k 1}$ for the UTs can be written as

$$
\begin{aligned}
\hat{\boldsymbol{H}}_{111} & =\left[\begin{array}{ll}
\boldsymbol{H}_{1111} & \boldsymbol{H}_{2111}
\end{array}\right], \\
\hat{\boldsymbol{H}}_{121} & =\left[\begin{array}{ll}
\boldsymbol{H}_{3121} & \boldsymbol{H}_{4121}
\end{array}\right], \\
\hat{\boldsymbol{H}}_{131} & =\left[\begin{array}{ll}
\boldsymbol{H}_{1131} & \boldsymbol{H}_{6131}
\end{array}\right] .
\end{aligned}
$$




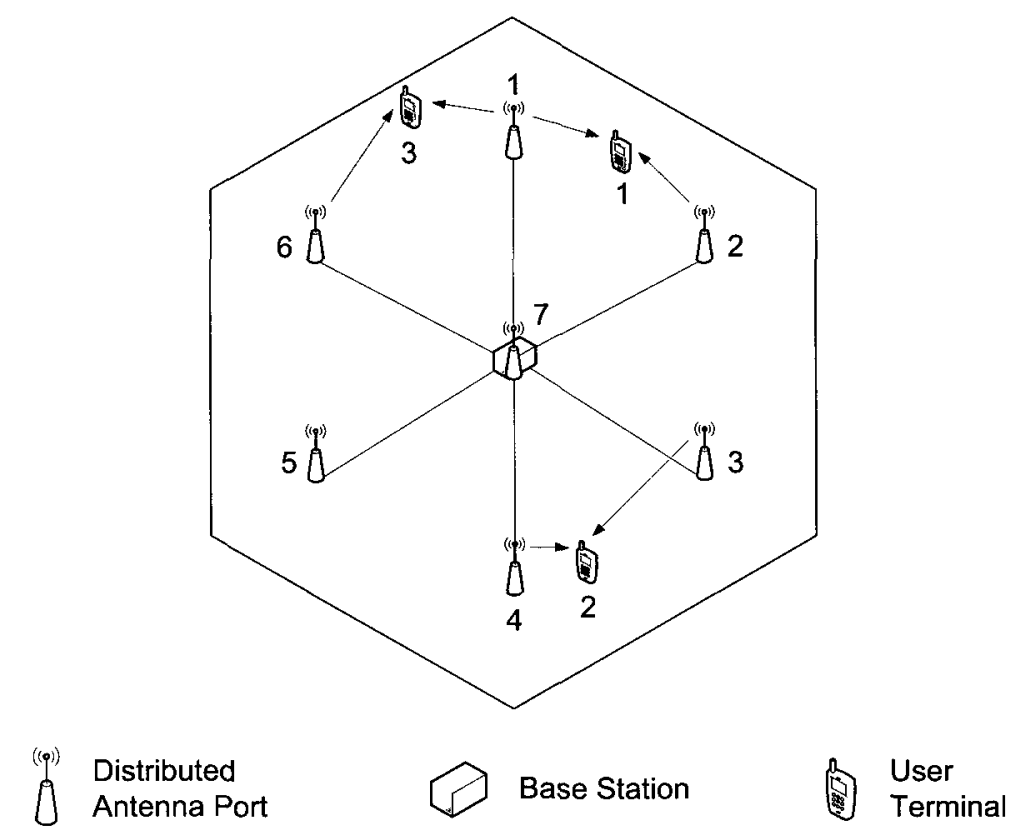

Figure 3.2: An exemplary single-cell DAS configuration with $K=3 \mathrm{UTs}$ per RB and $L=7$ ports.

In the case of $\mathrm{BD}$, the interference matrices are defined as

$$
\begin{gathered}
\tilde{\boldsymbol{H}}_{11}=\left[\begin{array}{c}
\overline{\boldsymbol{H}}_{121}^{\left(\mathcal{C}_{11}\right)} \\
\overline{\boldsymbol{H}}_{131}^{\left(\mathcal{C}_{11}\right)}
\end{array}\right]=\left[\begin{array}{ll}
\boldsymbol{H}_{1121} & \boldsymbol{H}_{2121} \\
\boldsymbol{H}_{1131} & \boldsymbol{H}_{2131}
\end{array}\right], \\
\tilde{\boldsymbol{H}}_{21}=\left[\begin{array}{c}
\overline{\boldsymbol{H}}_{111}^{\left(\mathcal{C}_{21}\right)} \\
\overline{\boldsymbol{H}}_{131}^{\left(\mathcal{C}_{21}\right)}
\end{array}\right]=\left[\begin{array}{ll}
\boldsymbol{H}_{3111} & \boldsymbol{H}_{4111} \\
\boldsymbol{H}_{3131} & \boldsymbol{H}_{4131}
\end{array}\right], \\
\tilde{\boldsymbol{H}}_{31}=\left[\begin{array}{c}
\overline{\boldsymbol{H}}_{111}^{\left(\mathcal{C}_{31}\right)} \\
\overline{\boldsymbol{H}}_{121}^{\left(\mathcal{C}_{31}\right)}
\end{array}\right]=\left[\begin{array}{ll}
\boldsymbol{H}_{1111} & \boldsymbol{H}_{6111} \\
\boldsymbol{H}_{1121} & \boldsymbol{H}_{6121}
\end{array}\right] .
\end{gathered}
$$

The corresponding BD beamforming submatrices $\hat{\boldsymbol{F}}_{k 1}$ then assume the form

$$
\begin{aligned}
\hat{\boldsymbol{F}}_{11} & =\left[\begin{array}{ll}
\boldsymbol{F}_{111}^{H} & \boldsymbol{F}_{211}^{H}
\end{array}\right]^{H}, \\
\hat{\boldsymbol{F}}_{21} & =\left[\begin{array}{ll}
\boldsymbol{F}_{321}^{H} & \boldsymbol{F}_{421}^{H}
\end{array}\right]^{H},
\end{aligned}
$$




$$
\hat{\boldsymbol{F}}_{31}=\left[\begin{array}{ll}
\boldsymbol{F}_{131}^{H} & \boldsymbol{F}_{631}^{H}
\end{array}\right]^{H}
$$

and the full BD beamforming matrices can be expressed as

$$
\begin{aligned}
& \boldsymbol{F}_{11}=\left[\begin{array}{lll}
\boldsymbol{F}_{111}^{H} & \boldsymbol{F}_{211}^{H} & \mathbf{0}_{3 N_{t} \times N_{r}}^{H}
\end{array}\right]^{H}, \\
& \boldsymbol{F}_{21}=\left[\begin{array}{llll}
\mathbf{0}_{2 N_{t} \times N_{r}}^{H} & \boldsymbol{F}_{321}^{H} & \boldsymbol{F}_{421}^{H} & \mathbf{0}_{N_{t} \times N_{r}}^{H}
\end{array}\right]^{H}, \\
& \boldsymbol{F}_{31}=\left[\begin{array}{lll}
\boldsymbol{F}_{131}^{H} & 0_{3 N_{t} \times N_{r}}^{H} & \boldsymbol{F}_{631}^{H}
\end{array}\right]^{H},
\end{aligned}
$$

where $\mathbf{0}_{a \times b}$ represents a $a \times b$ all-zero matrix.

For the ZF-DPC scheme, the interference matrices for the UTs can be written as

$$
\begin{gathered}
\tilde{\boldsymbol{H}}_{\pi(2) 1}=\overline{\boldsymbol{H}}_{\pi(1) 1}^{\left(\mathcal{C}_{\pi(2) 1}\right)}=\left[\begin{array}{ll}
\boldsymbol{H}_{31 \pi(1) 1} & \boldsymbol{H}_{41 \pi(1) 1}
\end{array}\right] \\
\tilde{\boldsymbol{H}}_{\pi(3) 1}=\left[\begin{array}{c}
\overline{\boldsymbol{H}}_{\pi(1) 1}^{\left(\mathcal{C}_{\pi(3) 1}\right)} \\
\overline{\boldsymbol{H}}_{\pi(2) 1}^{\left(\mathcal{C}_{\pi(3) 1}\right)}
\end{array}\right]=\left[\begin{array}{ll}
\boldsymbol{H}_{11 \pi(1) 1} & \boldsymbol{H}_{61 \pi(1) 1} \\
\boldsymbol{H}_{11 \pi(2) 1} & \boldsymbol{H}_{61 \pi(2) 1}
\end{array}\right],
\end{gathered}
$$

where $\boldsymbol{H}_{j m \pi(k) m}$ is the channel matrix between the $j$-th port in the $m$-th cell and the $\pi(k)$-th UT in this cell. The beamforming submatrices and full beamforming matrices for this scheme can be obtained analogously to the ones in (3.31)-(3.33) and (3.34)-(3.36), respectively.

\subsection{Centralized Multi-Cell Processing}

In this section, transmission schemes analogous to those in Section 3.3 will be developed under the assumption that the transmissions from the ports in all cells are fully coordinated by a central controller. Such a system can be viewed as a larger cell, which is hereby referred to as a super-cell. 


\subsubsection{System Model}

The system model in this section is analogous to that in Section 3.3.1, and hence, some repetitive details will be omitted. In the super-cell, which consists of a cluster of $M$ cells, the central controller is connected to $L M$ distributed $N_{t}$-antenna ports. In addition, there are $K M N_{r}$-antenna UTs in the super-cell, each of which can be served using any of the $L M$ ports ${ }^{5}$.

Let $\mathcal{S}$ represent the set of indices of all ports in the super-cell, where $|\mathcal{S}|=L M$. Let $\mathcal{A}$ represent the set of active ports in the super-cell $(\mathcal{A} \subseteq \mathcal{S})$. Also, let $\mathcal{C}_{k}$ denote the set of ports that transmit to the $k$-th UT in a coordinated manner, and let $\mathcal{I}_{k}$ denote the set of ports that cause interference to this UT, $k=1, \ldots, K M$.

\section{Signal Model}

The received signal, $\boldsymbol{y}_{k} \in \mathbb{C}^{N_{r}}$, of the $k$-th UT can be expressed as

$$
\boldsymbol{y}_{k}=\boldsymbol{H}_{k} \boldsymbol{x}+\boldsymbol{n}_{k}, \quad k=1, \ldots, K M,
$$

where, $\boldsymbol{H}_{k} \in \mathbb{C}^{N_{r} \times|\mathcal{A}| N_{t}}$ is the complex-valued channel matrix between all active ports in the super-cell and the $k$-th $\mathrm{UT}, \boldsymbol{n}_{k} \in \mathbb{C}^{N_{r}}$ is a zero-mean complex Gaussian noise vector with covariance matrix $E\left\{\boldsymbol{n}_{k} \boldsymbol{n}_{k}^{H}\right\}=\sigma^{2} \boldsymbol{I}_{N_{r}}$, and $\boldsymbol{x} \in \mathbb{C}^{|\mathcal{A}| N_{t}}$ is the signal transmitted from the active ports. This transmitted signal is of the form

$$
\boldsymbol{x}=\sum_{k=1}^{K M} \boldsymbol{F}_{k}^{\prime} \boldsymbol{u}_{k}
$$

where $\boldsymbol{u}_{k} \in \mathbb{C}^{N_{r}}$ denotes the data vector of the $k$-th $\mathrm{UT}$, and $\boldsymbol{F}_{k}^{\prime} \in \mathbb{C}^{|\mathcal{A}| N_{t} \times N_{r}}$ is its precoding matrix. Analogous to Section 3.3.1, this precoding matrix is chosen to be

\footnotetext{
${ }^{5}$ To enable a fair comparison between the single-cell processing schemes and the corresponding centralized multi-cell processing schemes, $K$ UTs are randomly located in each of the $M$ cells that comprise the super-cell.
} 
of the form $\boldsymbol{F}_{k}^{\prime}=\boldsymbol{F}_{k} \boldsymbol{\Lambda}_{k}^{\frac{1}{2}}$, where $\boldsymbol{F}_{k} \in \mathbb{C}^{|\mathcal{A}| N_{t} \times N_{r}}$ is the transmit beamforming matrix and $\boldsymbol{\Lambda}_{k} \in \mathbb{C}^{N_{r} \times N_{r}}$ is a diagonal power allocation matrix.

The ports in the super-cell are subject to a total power constraint, $P_{t} M$; that is, $E\left\{\boldsymbol{x} \boldsymbol{x}^{H}\right\} \leq P_{t} M$. Assuming that the data vectors, $\boldsymbol{u}_{k}$, are zero-mean with identity covariance matrix for all $k$, this constraint can be expressed as

$$
\sum_{k=1}^{K M} \operatorname{Tr}\left(\boldsymbol{S}_{k}\right) \leq P_{t} M
$$

where $\boldsymbol{S}_{k}=\boldsymbol{F}_{k}^{\prime} \boldsymbol{F}_{k}^{\prime H}$ is the transmit covariance matrix of the $k$-th UT.

\section{Channel Model}

The channel matrix $\boldsymbol{H}_{k}$ in the signal model above can be expressed as

$$
\boldsymbol{H}_{k}=\left[\begin{array}{llll}
\boldsymbol{H}_{1 k} & \boldsymbol{H}_{2 k} & \ldots & \boldsymbol{H}_{|\mathcal{A}| k}
\end{array}\right], \quad k=1, \ldots, K M
$$

where

$$
\boldsymbol{H}_{\ell k}=\sqrt{\rho\left(d_{\ell k}\right) s_{\ell k}} \boldsymbol{H}_{\ell k}^{\prime}
$$

In $(3.43), d_{\ell k}$ is the distance between the $\ell$-th port and the $k$-th UT, $s_{\ell k}$ represents log-normal shadowing with $0 \mathrm{~dB}$ mean and standard deviation $\sigma_{s}$ in $\mathrm{dB}$, and $\boldsymbol{H}_{\ell k}^{\prime} \in$ $\mathbb{C}^{N_{r} \times N_{t}}$ represents Rayleigh fading.

\subsubsection{DAS Block Diagonalization}

In this section, the goal is to design the precoding matrices of the UTs such that interuser interference is mitigated. It is noted that in the case of single-cell processing in Section 3.3.2, the number of UTs per cell in a particular RB is chosen such that the DAS dimensionality constraint in (3.17) is satisfied. However, for a system in which the values of $N_{t}$ and $N_{r}$, as well as the number of coordinating ports per UT are 
preserved, this constraint is generally not satisfied in the multi-cell processing case. In other words, there are not enough spatial degrees of freedom to transmit to the $k$-th UT such that this transmission is in the null space of the channels to all the other $K M-1$ UTs. Hence, the interference caused to only a subset of these UTs may be mitigated while satisfying the dimensionality constraint. A candidate approach is to select those $K-1$ UTs that have the largest channel gains from the ports that transmit to the $k$-th UT.

Let $\varphi_{k}$ denote a permutation operator for UT ordering, whereby the $K M-1$ UTs (excluding the $k$-th UT) are arranged in decreasing order of the magnitude of the channel gains from the ports that transmit to the $k$-th UT. In other words, $\varphi_{k}(1)=\max _{\jmath=1,}, K M, \jmath \neq k\left\|\overline{\boldsymbol{H}}_{\jmath}^{\left(\mathcal{C}_{k}\right)}\right\|_{2}$, and $\varphi_{k}(K M-1)=\min _{\jmath=1,, K M, \jmath \neq k}\left\|\overline{\boldsymbol{H}}_{\jmath}^{\left(\mathcal{C}_{k}\right)}\right\|_{2}$, where $\overline{\boldsymbol{H}}_{\jmath}^{\left(\mathcal{C}_{k}\right)} \in \mathbb{C}^{N_{r} \times\left|\mathcal{C}_{k}\right| N_{t}}$ is a submatrix containing only those columns of $\boldsymbol{H}_{\jmath}$ that correspond to the antennas of the ports in $\mathcal{C}_{k}$, and $\|\cdot\|_{2}$ denotes the Frobenius norm. Using this notation, the interference matrix of the $k$-th UT is defined as

$$
\tilde{\boldsymbol{H}}_{k} \triangleq\left[\begin{array}{lll}
\overline{\boldsymbol{H}}_{\varphi_{k}(1)}^{\left(\mathcal{C}_{\mathcal{H}) H}\right.} & \ldots & \overline{\boldsymbol{H}}_{\varphi_{k}(K-1)}^{\left(\mathcal{C}_{k}\right) H}
\end{array}\right]^{H}, \quad k=1, \ldots, K M
$$

From this point onward, the design of the beamforming and power allocation matrices is similar to that in Section 3.3.2, and hence, it is omitted here for conciseness.

Let $\mathcal{U}_{k}$ denote the set of UTs whose beamforming matrices are designed to mitigated interference to the $k$-UT, for all $k$. Then the average aggregate spectral efficiency per cell per RB can be expressed as

$$
R_{\mathrm{BD}}=\frac{1}{M} \sum_{k=1}^{K M} \log _{2} \frac{\left|\sigma^{2} \boldsymbol{I}_{N_{r}}+\sum_{\jmath \in \overline{\mathcal{U}}_{k} \backslash\{k\}}\left(\hat{\boldsymbol{U}}_{k}^{H} \boldsymbol{H}_{k} \boldsymbol{F}_{\jmath} \boldsymbol{\Lambda}_{\jmath}^{\frac{1}{2}}\right)^{2}+\left(\hat{\boldsymbol{U}}_{k}^{H} \boldsymbol{H}_{k} \boldsymbol{F}_{k} \boldsymbol{\Lambda}_{k}^{\frac{1}{2}}\right)^{2}\right|}{\left|\sigma^{2} \boldsymbol{I}_{N_{r}}+\sum_{\jmath \in \overline{\mathcal{U}}_{k} \backslash\{k\}}\left(\hat{\boldsymbol{U}}_{k}^{H} \boldsymbol{H}_{k} \boldsymbol{F}_{\jmath} \boldsymbol{\Lambda}_{\jmath}^{\frac{1}{2}}\right)^{2}\right|},
$$

where $\{\cdot \bar{\cdot}\}$ denotes the complement of the set argument, and $\{\cdot\} \backslash\{\cdot\}$ is the set difference operator. 


\subsubsection{DAS Zero-Forcing Dirty Paper Coding}

The DAS ZF-DPC scheme in the case of centralized multi-cell processing is analogous to that developed in Section 3.3.3 with modifications that are similar to those used in Section 3.4.2. In particular, the $K M$ UTs are arranged in a particular order, which is denoted by the permutation operator $\pi$. The data vector of the $\pi(k)$-th UT, $\boldsymbol{u}_{\pi(k)}$, is generated using successive dirty paper encoding, such that this UT does not experience interference from the UTs with a lower index. The remaining interference is partially mitigated using the approach described in Section 3.4.2.

Let $\varphi_{\pi(k)}$ denote another UT ordering permutation operator, whereby the $K M-$ $k-1$ UTs that have a lower $\pi$-index are arranged in decreasing order of the magnitude of the channel gains from the ports that transmit to the $\pi(k)$-th UT. Using this notation, the interference matrix of the $\pi(k)$-th UT is defined as

$$
\tilde{\boldsymbol{H}}_{\pi(k)} \triangleq\left[\begin{array}{lll}
\overline{\boldsymbol{H}}_{\varphi_{k}(1)}^{\left(\mathcal{C}_{\pi(k)}\right) H} & \ldots & \overline{\boldsymbol{H}}_{\varphi_{k}(K-1)}^{\left(\mathcal{C}_{\pi(k)}\right) H}
\end{array}\right]^{H}, \quad k=2, \ldots, K M
$$

The remaining steps of the precoding matrix design are similar to those in Sec-

tion 3.4.2. Now, the average aggregate spectral efficiency per cell per RB can be expressed as

$$
R_{\mathrm{ZF}-\mathrm{DPC}}=\frac{1}{M} \max _{\pi} \sum_{k=1}^{K M} \log _{2} \frac{\left|\boldsymbol{\Xi}+\left(\hat{\boldsymbol{U}}_{\pi(k)}^{H} \boldsymbol{H}_{\pi(k)} \boldsymbol{F}_{\pi(k)} \boldsymbol{\Lambda}_{\pi(k)}^{\frac{1}{2}}\right)^{2}\right|}{|\boldsymbol{\Xi}|}
$$

where

$$
\boldsymbol{\Xi}=\sigma^{2} \boldsymbol{I}_{N_{r}}+\sum_{\jmath>k, \jmath \in \overline{\mathcal{U}}_{\pi(k)} \backslash\{\pi(k)\}}\left(\hat{\boldsymbol{U}}_{\pi(k)}^{H} \boldsymbol{H}_{\pi(k)} \boldsymbol{F}_{\pi(\jmath)} \boldsymbol{\Lambda}_{\pi(\jmath)}^{\frac{1}{2}}\right)^{2}
$$

and $\mathcal{U}_{\pi(k)}$ is analogous to $\mathcal{U}_{k}$ in Section 3.4.2. 


\subsection{Performance Evaluation}

The performance of the cellular DAS transmission schemes developed in this chapter is assessed using Monte Carlo simulation, and is compared with that of the corresponding schemes in a cellular CAS. To enable a fair comparison, the same total power constraint is applied to each cell in both architectures. As well, the number of active antennas that transmit to each UT in the DAS, is preserved in the case of the CAS by selecting those $\left|\mathcal{C}_{k m}\right| N_{t}$ antennas out of the total $\left|\mathcal{A}_{m}\right| N_{t}$ available ones in the CAS that have the largest channel gains (including both large-scale and small-scale fading) to the $k$-th UT in the $m$-th cell.

The cellular system used for the Monte Carlo simulation consists of $M$ hexagonal cells, each with circumradius $r_{c}$. The BS in each cell is connected to seven ports; i.e., $L=7[53,54]$. To evenly cover the geographic area of the cell, six of these ports are located uniformly at a distance of $\frac{2}{3} r_{c}$ from the BS at the center of the cell, while the seventh port is co-located with the BS (see, e.g., Fig. 3.1) ${ }^{6}$. There are $K=3$ UTs per RB in each cell, and each UT is equipped with $N_{r}=2$ antennas. The results reported in this section are averaged over 10000 independent channel realizations. In each realization, the UTs are dropped randomly in each cell.

To simulate practical communication scenarios, the selected values for the the distance and the log-normal shadowing and path loss parameters are those corresponding to the suburban macro-cell (SMa) IMT-Advanced scenario [57, Sections 8.4.2, A-1.3.1]. For this scenario, the distance between the BSs is $1299 \mathrm{~m}$, and the corresponding shadowing standard deviation, $\sigma_{s}$, is $8 \mathrm{~dB}$. Using an RB bandwidth of $180 \mathrm{kHz}$ [58], and a thermal noise level and UT noise figure of $-174 \mathrm{dBm} / \mathrm{Hz}$ and $7 \mathrm{~dB}$, respectively [57], the noise power, $\sigma^{2}$, is chosen to be $-114 \mathrm{dBm}$. The channel model is chosen to be the non-line-of-sight (NLoS) one in [57]. In this model, setting

\footnotetext{
${ }^{6}$ The described configuration is chosen for simplicity; the location of the ports is a separate optimization problem (see, e.g. $[55,56]$ ), which is not considered herein.
} 
Table 3.1: System and simulation parameters used for the cellular DAS architecture

\begin{tabular}{|l|c|}
\hline Parameter & Value \\
\hline UTs per cell per RB, $K$ & 3 \\
Antennas at each UT, $N_{r}$ & 2 \\
Ports per cell, $L$ & 7 \\
Noise power, $\sigma^{2}$ & $-114 \mathrm{dBm}$ \\
Inter-BS distance & $1299 \mathrm{~m}$ \\
Elevation of each port & $15 \mathrm{~m}$ \\
Elevation of each UT & $1.5 \mathrm{~m}$ \\
Carrier frequency & $2 \mathrm{GHz}$ \\
Path loss and shadowing & $\mathrm{NLoS}[57]$ \\
Log-normal shadowing standard deviation, $\sigma_{s}$ & $8 \mathrm{~dB}$ \\
Independent channel realizations & 10000 \\
\hline
\end{tabular}

the carrier frequency to $2 \mathrm{GHz}$, the elevation of each antenna port to $15 \mathrm{~m}$, and the elevation of each UT to $1.5 \mathrm{~m}$ yields the following path loss function:

$$
\rho(d)=10^{1.866+4.032 \log _{10}(d)} .
$$

The system and simulation parameters described above are summarized in Table 3.1. All numerical results shown in this section are generated using MATLAB.

\subsubsection{Single-Cell System (Interference-Free Environment)}

In this section, the performance evaluation is carried out for a system comprised of a single cell (i.e., $M=1$ ) which operates in an isolated (interference-free) environment. The chosen performance metric is the aggregate cell spectral efficiency per RB in bits/sec/Hz. The results presented herein serve as an upper bound on the achievable aggregate cell spectral efficiency in a more realistic multi-cell system, which is considered in Section 3.5.2. 


\section{Port Selection versus Transmit Antenna Selection}

Port selection can be viewed as a special case of transmit antenna selection, wherein a subset of all transmit antennas are selected to transmit to a particular UT based on a specified metric such as the channel gain or the received SINR. However, with port selection, the antennas at each port are selected as a group. The performance of both approaches is compared in Examples 3.1 and 3.2 below with antenna selection referring to the selection of $\left|\mathcal{C}_{k m}\right| N_{t}$ antennas with the largest channel gains (including both large-scale and small-scale fading) to the $k$-th UT out of the total $\left|\mathcal{A}_{m}\right| N_{t}$ antennas. Unlike antenna selection, however, the criterion used for port selection in the simulations reported herein is large-scale fading (path loss and shadowing) only.

In Figs. 3.3 and 3.4, it is shown that although port selection is less flexible, its performance is comparable to that of antenna selection for both DAS ZF-DPC and DAS BD. Hence, antenna selection in the DAS is not considered beyond this initial comparison in this section.

Example 3.1. In Fig. 3.3(a), the ergodic aggregate cell spectral efficiency per RB achieved by DAS BD and DAS ZF-DPC is compared with those achieved by the CAS counterparts of these schemes ${ }^{7}$. In Fig. 3.3(b), the outage probability of these schemes is shown versus the aggregate cell spectral efficiency per RB when $P_{t}=20 \mathrm{dBm}$. In this scenario, $\left|\mathcal{C}_{k m}\right|=3$ for all $k$ and $N_{t}=2$. The DAS dimensionality constraint is satisfied here because $N_{r}(K-1)=4<\min _{k \in\{1, \ldots, K\}}\left|\mathcal{C}_{k m}\right| N_{t}=6$. However, no more UTs could be simultaneously served by this system while satisfying the zero-forcing condition unless either additional antennas were deployed at each port or a greater number of ports coordinated their transmissions to each UT.

It can be seen from the figure that both DAS schemes achieve higher aggregate cell spectral efficiencies than their CAS counterparts. For example, when $P_{t}=20 \mathrm{dBm}$ in

\footnotetext{
${ }^{7}$ Each of these spectral efficiencies is obtained by evaluating the corresponding expressions (see, e.g., (3.20) and (3.23)) for each independent channel realization, and then averaging the results over all of the realizations.
} 


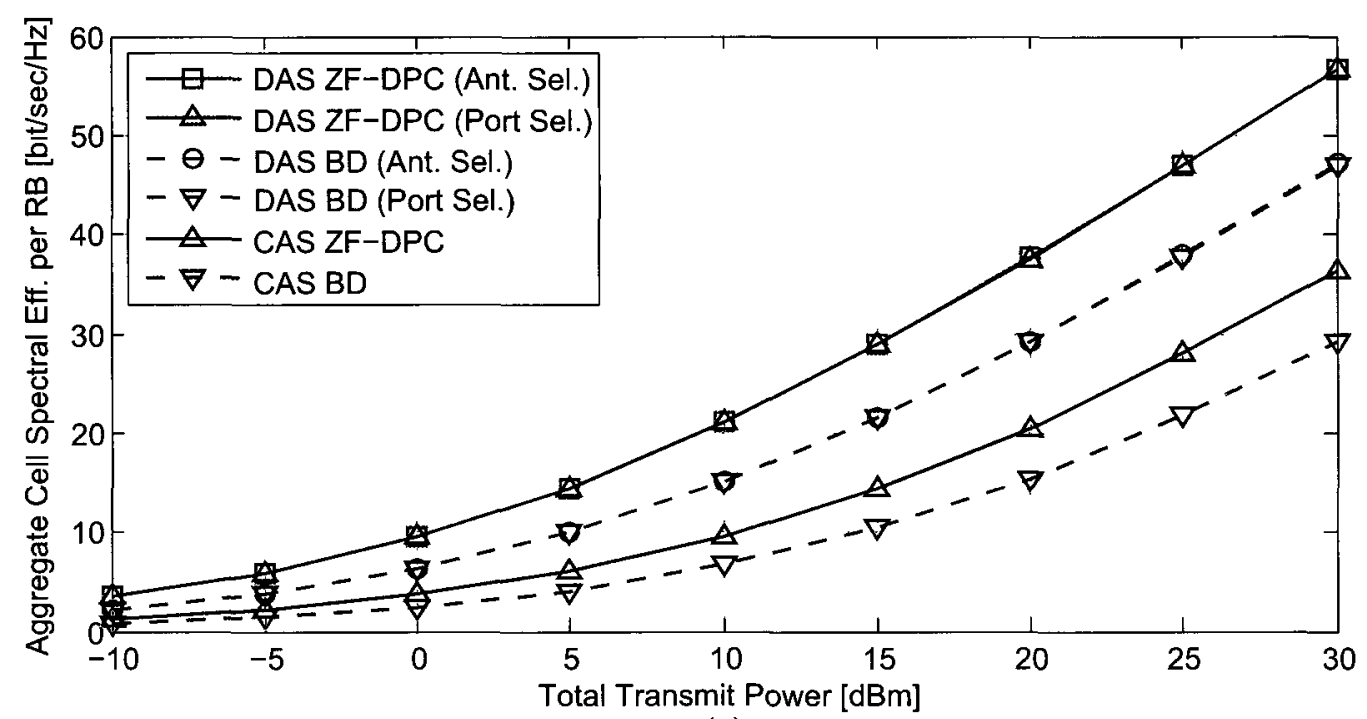

(a)

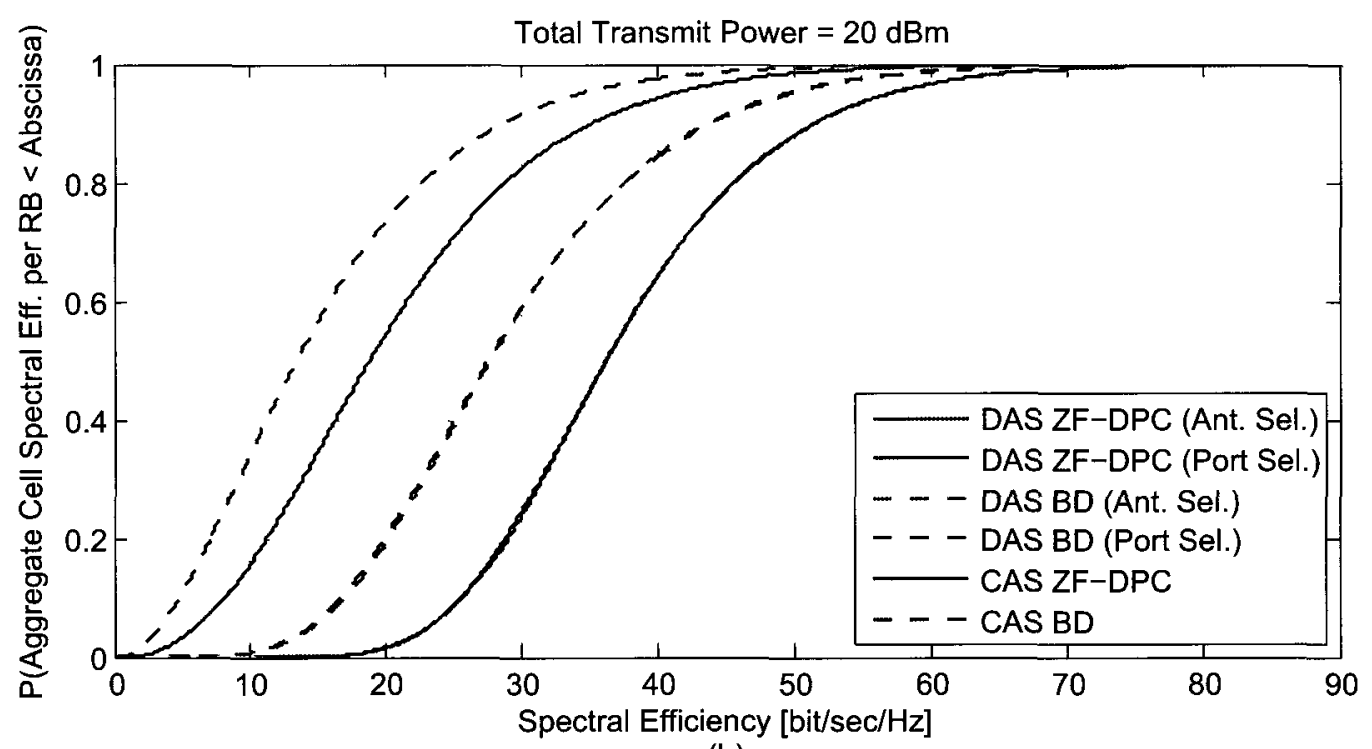

(b)

Figure 3.3: A comparison between the ergodic and outage aggregate cell spectral efficiencies per RB achieved by DAS BD and DAS ZF-DPC and those achieved by their CAS counterparts in a single-cell system that operates in an interference-free environment. $N_{t}=2$ and $\left|\mathcal{C}_{k m}\right|=3, \forall k$. 
Fig. 3.3(a), each DAS scheme achieves over $80 \%$ higher ergodic aggregate cell spectral efficiency per RB than the corresponding CAS scheme. Moreover, DAS ZF-DPC outperforms DAS BD by approximately $28 \%$. This gain is due to the availability of additional spatial degrees of freedom in the case of DAS ZF-DPC since the interference is partially mitigated through successive DPC encoding. Hence, the zero-forcing condition must only be satisfied for the remaining interference.

Example 3.2. In Fig. 3.4, a comparison similar to the one in Fig. 3.3 is considered, except that now $N_{t}=3$. It can be seen from this figure that, in comparison to the case of $N_{t}=2$ considered in Example 3.1, a single additional antenna at each port results in improved performance for both DAS schemes. Furthermore, the gap between both DAS schemes is reduced. For example, when $P_{t}=20 \mathrm{dBm}$, DAS ZFDPC only achieves approximately $9 \%$ higher ergodic aggregate spectral efficiency than DAS BD, which is considerably less than the corresponding result in Example 3.1.

Example 3.3. In Fig. 3.5, a comparison similar to the one in Fig. 3.3 is considered, except that now $\left|\mathcal{C}_{k m}\right|=7, \forall k$. The observation made from comparing the two figures is similar to that in Example 3.2. It can be seen that by using all $L=7$ ports to serve each UT leads to improved performance for both DAS schemes, and that the gap between DAS ZF-DPC and DAS BD is reduced. This improvement can be attributed to the additional spatial degrees of freedom that are made available by increasing $\left|\mathcal{C}_{k m}\right|$ from 3 to 7 for all $k$.

\section{Effect of the Number of Antennas per Port}

Example 3.4. Following the lead from Examples 3.1 and 3.2, in this example, the effect the number of antennas per port is further investigated. In Fig. 3.6, the aggregate cell spectral efficiencies per RB achieved by both the DAS and CAS schemes are compared for different values of $N_{t}$ when $P_{t}=20 \mathrm{dBm}$ and $\left|\mathcal{C}_{k m}\right|=3$ for all $k$. It can be noted from the figure that the points at $N_{t}=2$ and $N_{t}=3$ are the same 


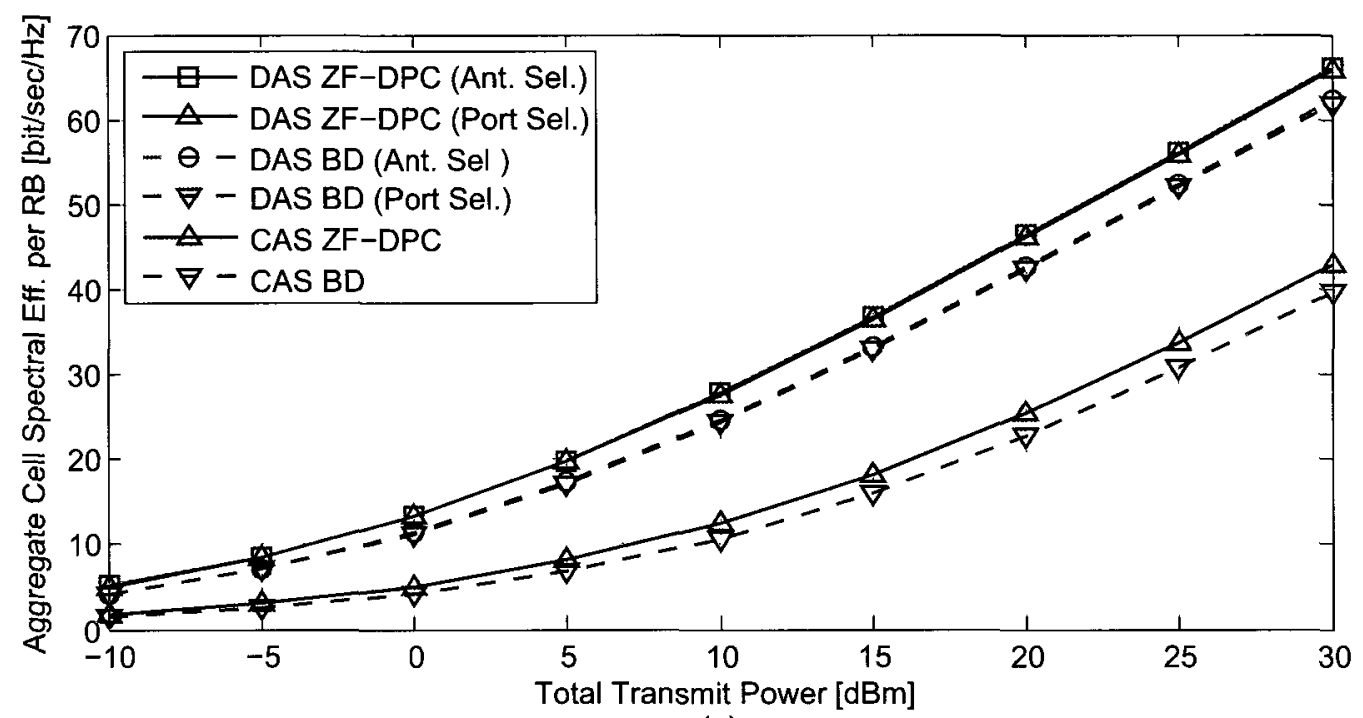

(a)

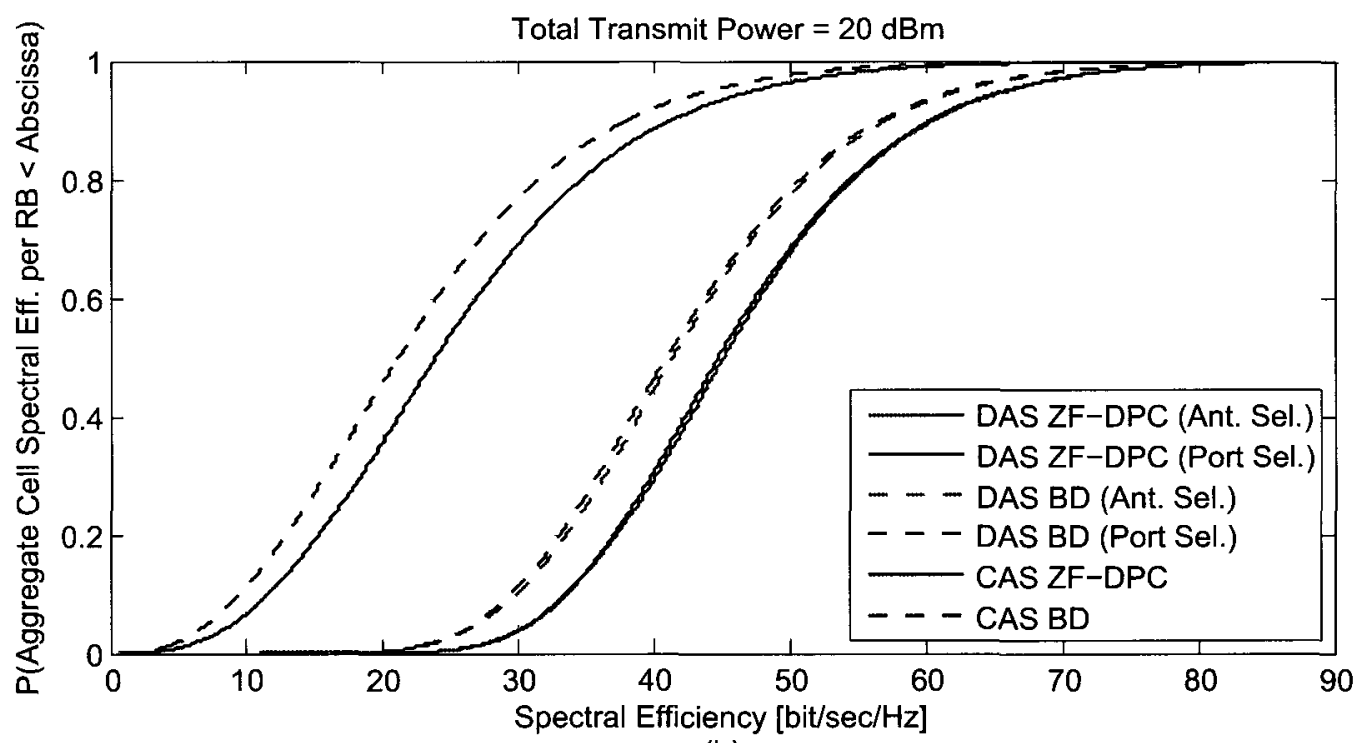

(b)

Figure 3.4: A comparison between the ergodic and outage aggregate cell spectral efficiencies per RB achieved by DAS BD and DAS ZF-DPC and those achieved by their CAS counterparts in a single-cell system that operates in an interference-free environment. $N_{t}=3$ and $\left|\mathcal{C}_{k m}\right|=3, \forall k$. 


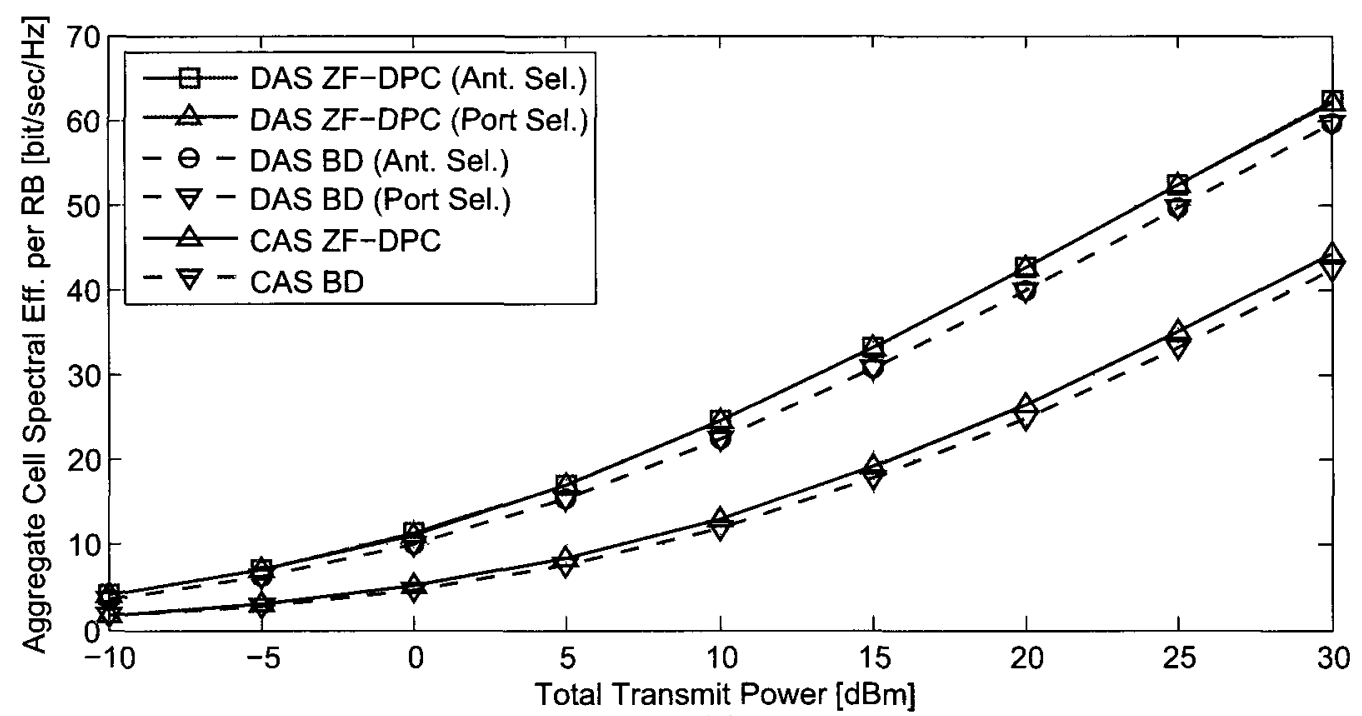

(a)

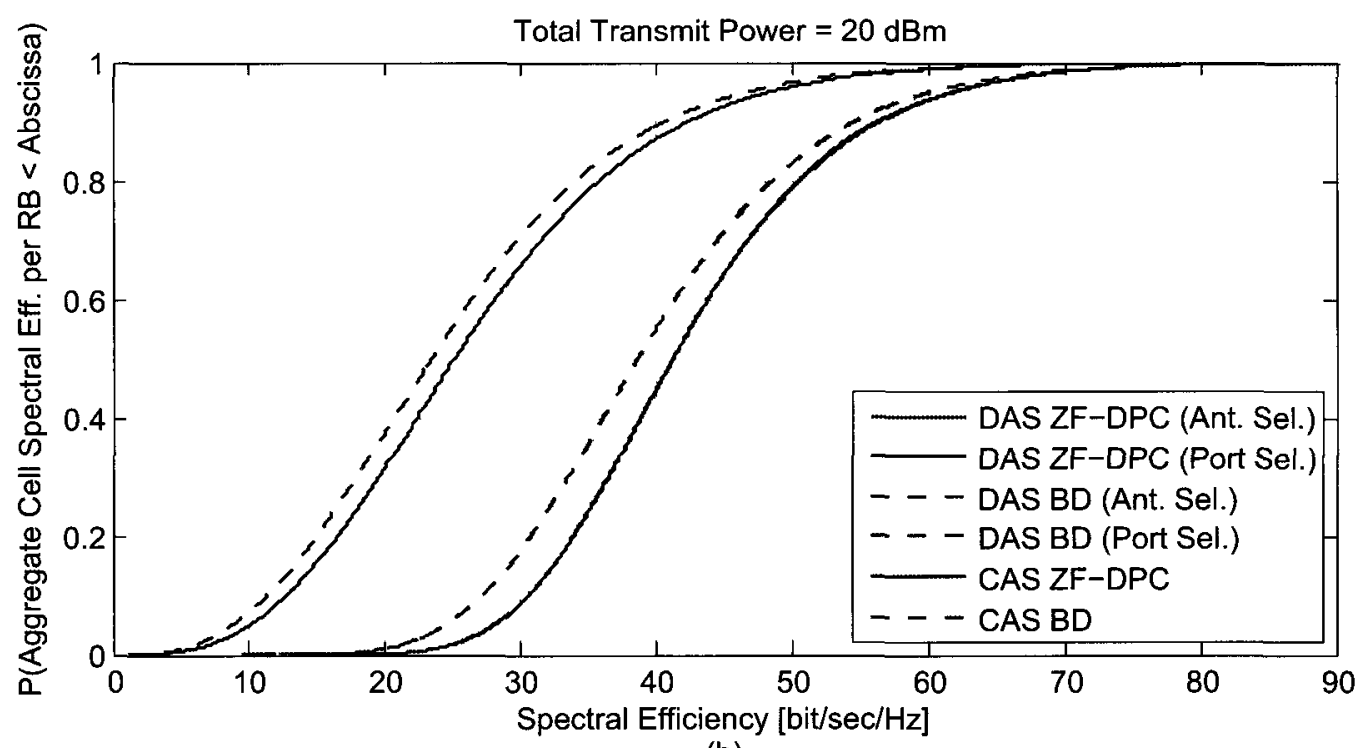

(b)

Figure 3.5: A comparison between the ergodic and outage aggregate cell spectral efficiencies per RB achieved by DAS BD and DAS ZF-DPC and those achieved by their CAS counterparts in a single-cell system that operates in an interference-free environment. $N_{t}=2$ and $\left|\mathcal{C}_{k m}\right|=7, \forall k$. 


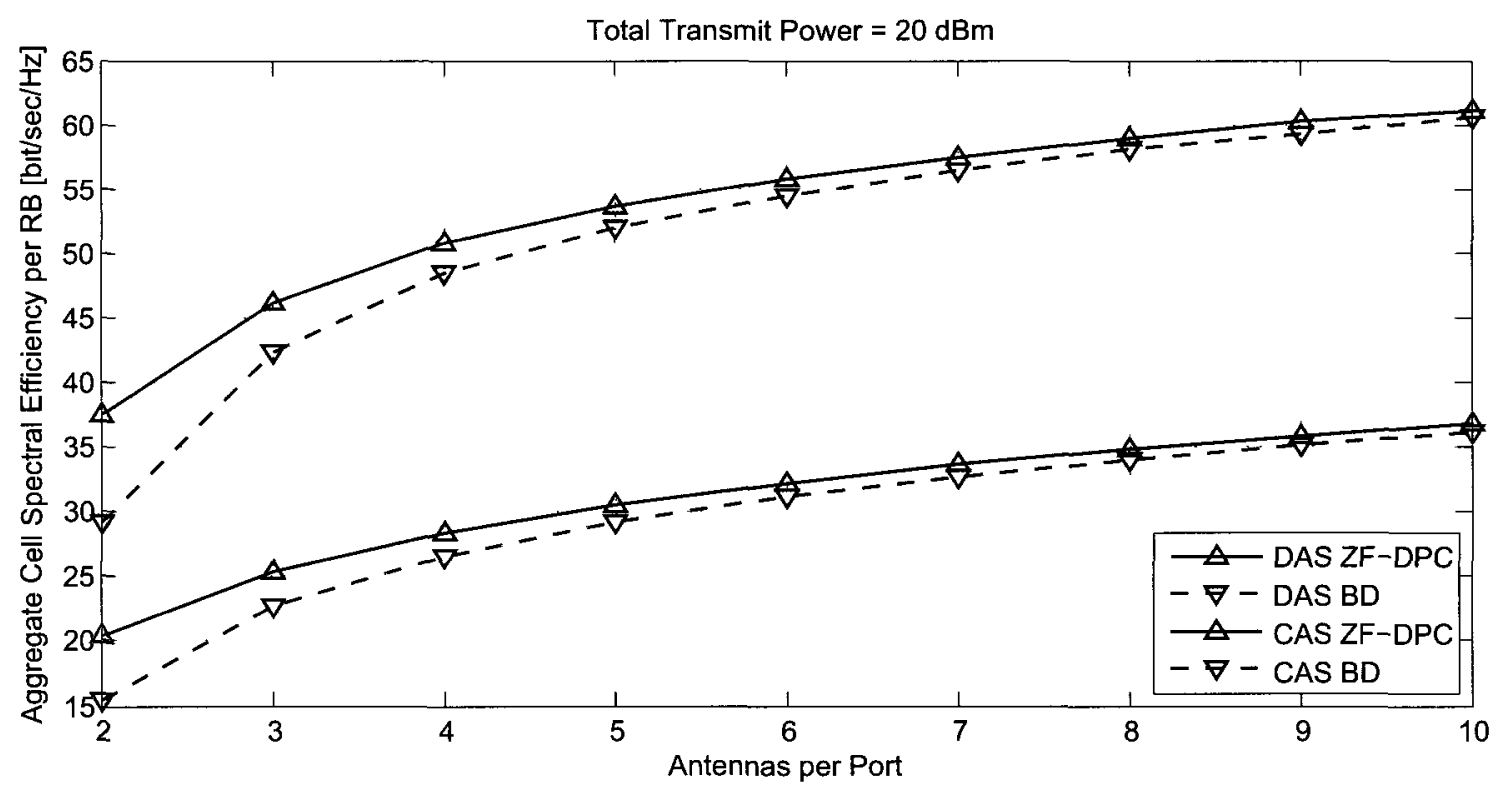

Figure 3.6: A comparison between the aggregate cell spectral efficiencies per RB achieved by DAS BD and DAS ZF-DPC and those achieved by their CAS counterparts for different values of $N_{t}$ in a single-cell system that operates in an interference-free environment. $\left|\mathcal{C}_{k m}\right|=3, \forall k$.

as those in Figs. 3.3(a) and 3.4(a) for $P_{t}=20 \mathrm{dBm}$, respectively. Furthermore, it can be observed that as $N_{t}$ increases, the spectral efficiency achieved by each of the schemes also increases. This improvement can be attributed to the availability of additional spatial degrees of freedom since the DAS dimensionality constraint is more than barely satisfied beyond $N_{t}=2$. However, it can be seen from the figure that the improvement diminishes as the number of antennas per port becomes large.

\section{Effect of the Number of Coordinating Ports per UT}

Example 3.5. Following the lead from Example 3.3, in this example, the effect of varying the value of $\left|\mathcal{C}_{k m}\right|$ is investigated, and the corresponding simulation results are presented in Fig. 3.7. For this simulation, $N_{t}$ is fixed as 3, and the lowest chosen value of $\left|\mathcal{C}_{k m}\right|$ is 2 so that the zero-forcing constraint is satisfied.

The observation in Fig. 3.7 is analogous to the one in Example 3.4. In particular, the aggregate cell spectral efficiency per RB achieved by both DAS schemes is rela- 


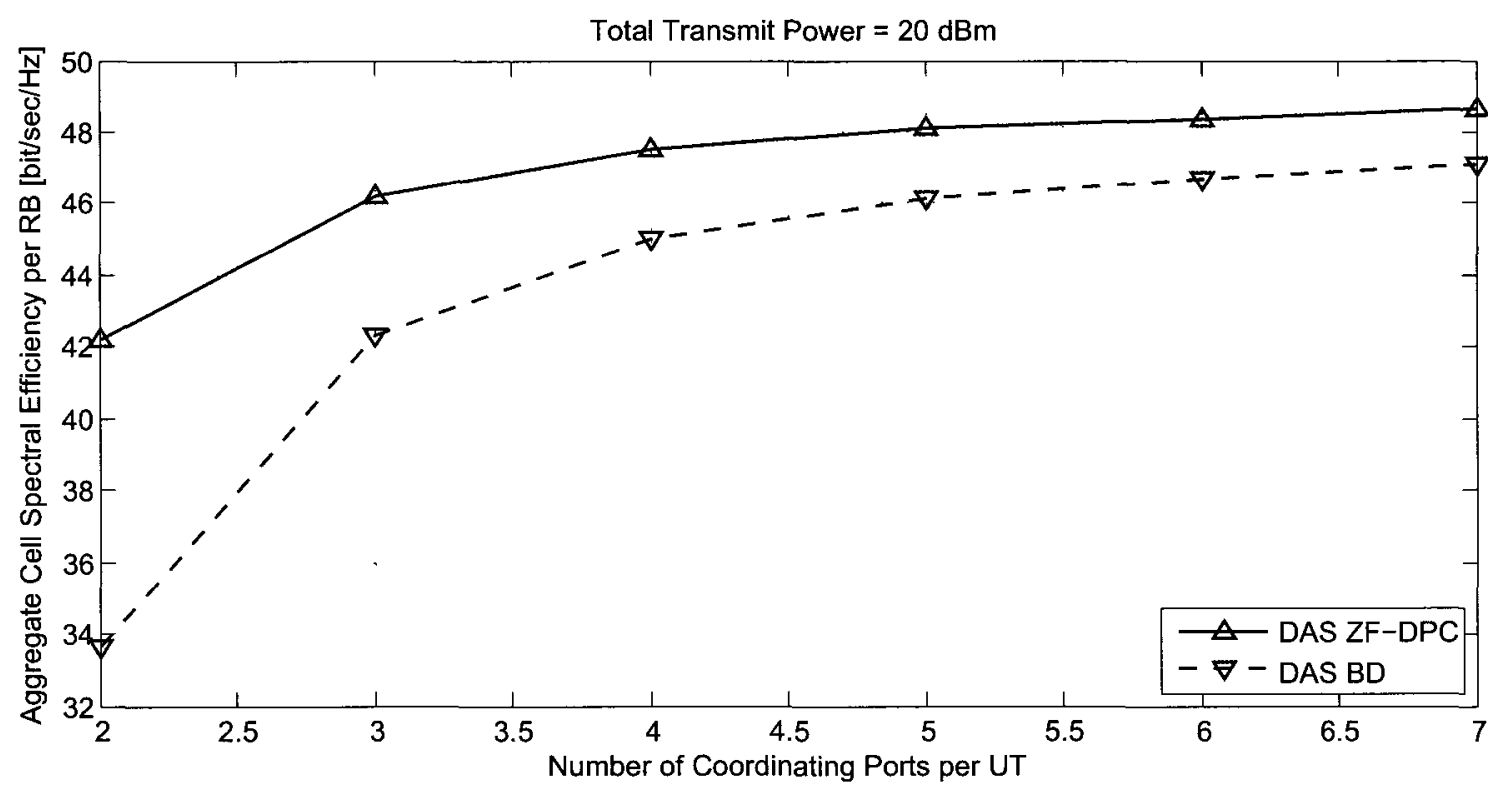

Figure 3.7: A comparison between the aggregate cell spectral efficiencies per RB achieved by DAS BD and DAS ZF-DPC for different values of $\left|\mathcal{C}_{k m}\right|$ in a single-cell system that operates in an interference-free environment. $N_{t}=3$.

tively low when $\left|\mathcal{C}_{k m}\right|=2$, and it increases monotonically with $\left|\mathcal{C}_{k m}\right|$. However, this improvement diminishes as $\left|\mathcal{C}_{k m}\right|$ approaches $L$. This monotonically increasing behaviour is due to a larger number of spatial degrees of freedom that become available as $\left|\mathcal{C}_{k m}\right|$ increases for all $k$.

\section{Effect of UT distance from the Cell Center}

Example 3.6. In this example, the performance of the proposed DAS schemes is compared with that of their CAS counterparts for different UT locations in the cell area. In particular, each of the $K$ UTs is dropped randomly on a circle with radius $r$ that is centered at the BS. The value of $r$ is progressively increased on the interval $\left[10, r_{c}\right) \mathrm{m}^{8}$. In doing so, it is demonstrated that, in contrast to the CAS, the DAS architecture enables more ubiquitous high-throughput coverage throughout the cell.

In Fig. 3.8, the aggregate cell spectral efficiency per RB achieved by DAS ZF-

\footnotetext{
${ }^{8}$ The value of $r$ is lower-bounded by $10 \mathrm{~m}$ since the chosen path loss model is not applicable when the receivers are located too close to the transmitter.
} 


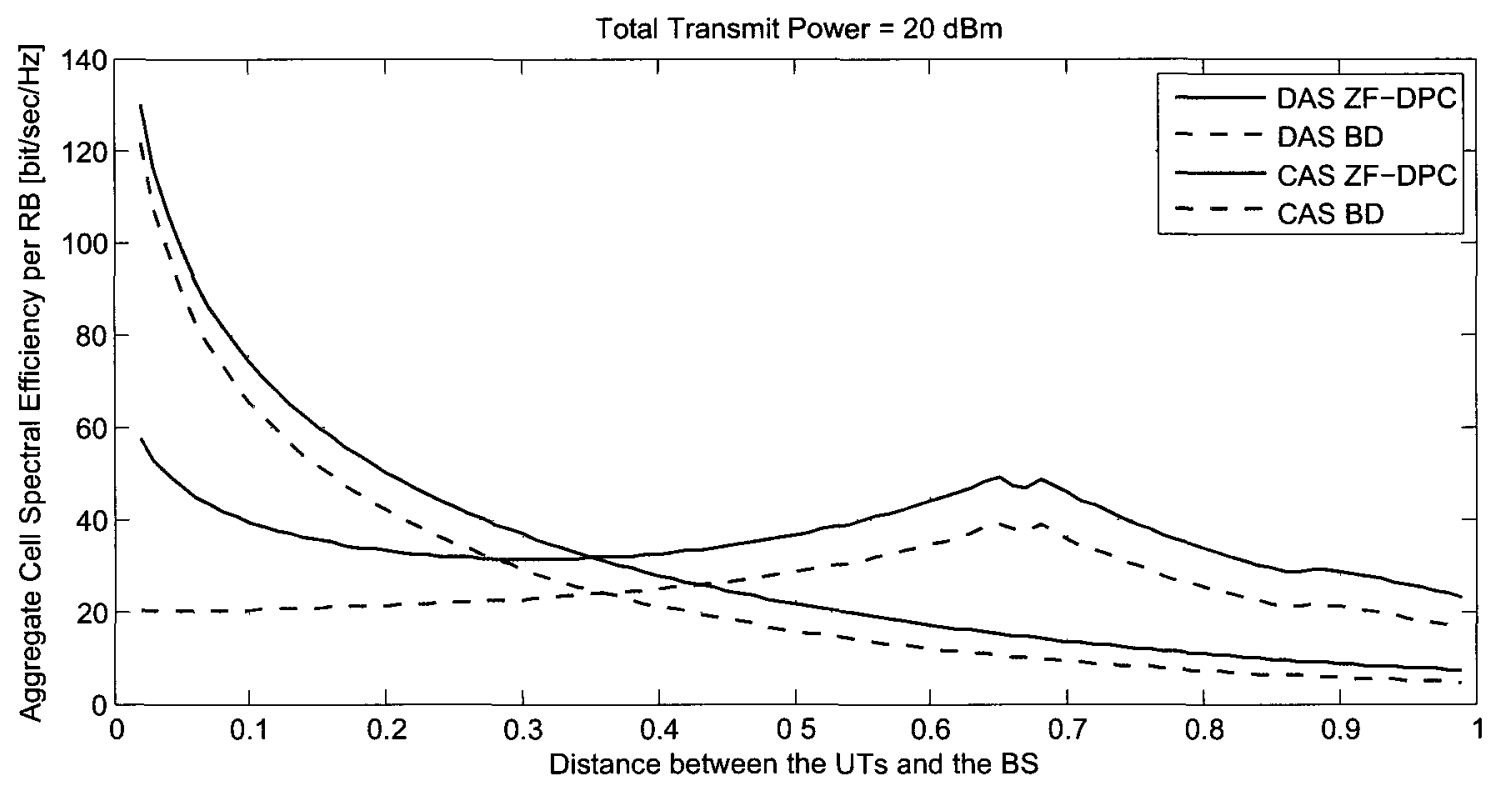

Figure 3.8: A comparison between the aggregate cell spectral efficiencies per RB achieved by DAS BD and DAS ZF-DPC, and those achieved by their CAS counterparts for different UT locations in a single-cell system that operates in an interferencefree environment. $N_{t}=2$ and $\left|\mathcal{C}_{k m}\right|=3, \forall k$.

DPC is compared with that achieved by DAS BD, and that achieved by the CAS counterparts of these two schemes for different values of $r$ on the interval specified above when $P_{t}=20 \mathrm{dBm}$ and $\left|\mathcal{C}_{k m}\right|=3$ for all $k$. It can be seen from the figure that, as expected, the spectral efficiency achieved by the CAS schemes is high near the cell center, and decreases exponentially as $r$ increases. In contrast, it can be observed that the spectral efficiency achieved by the DAS schemes increases as the distance approaches $\frac{2}{3} r_{c}$. This is because six of the seven ports are located on a circle of this radius, which allows the UTs to benefit from the higher probability of being geographically close to a subset of these ports.

\subsubsection{Multi-Cell System with Single-Cell Processing}

In Section 3.5.1, the performance of the proposed DAS transmission schemes was assessed for a single cell that operates in an interference-free environment. In this 


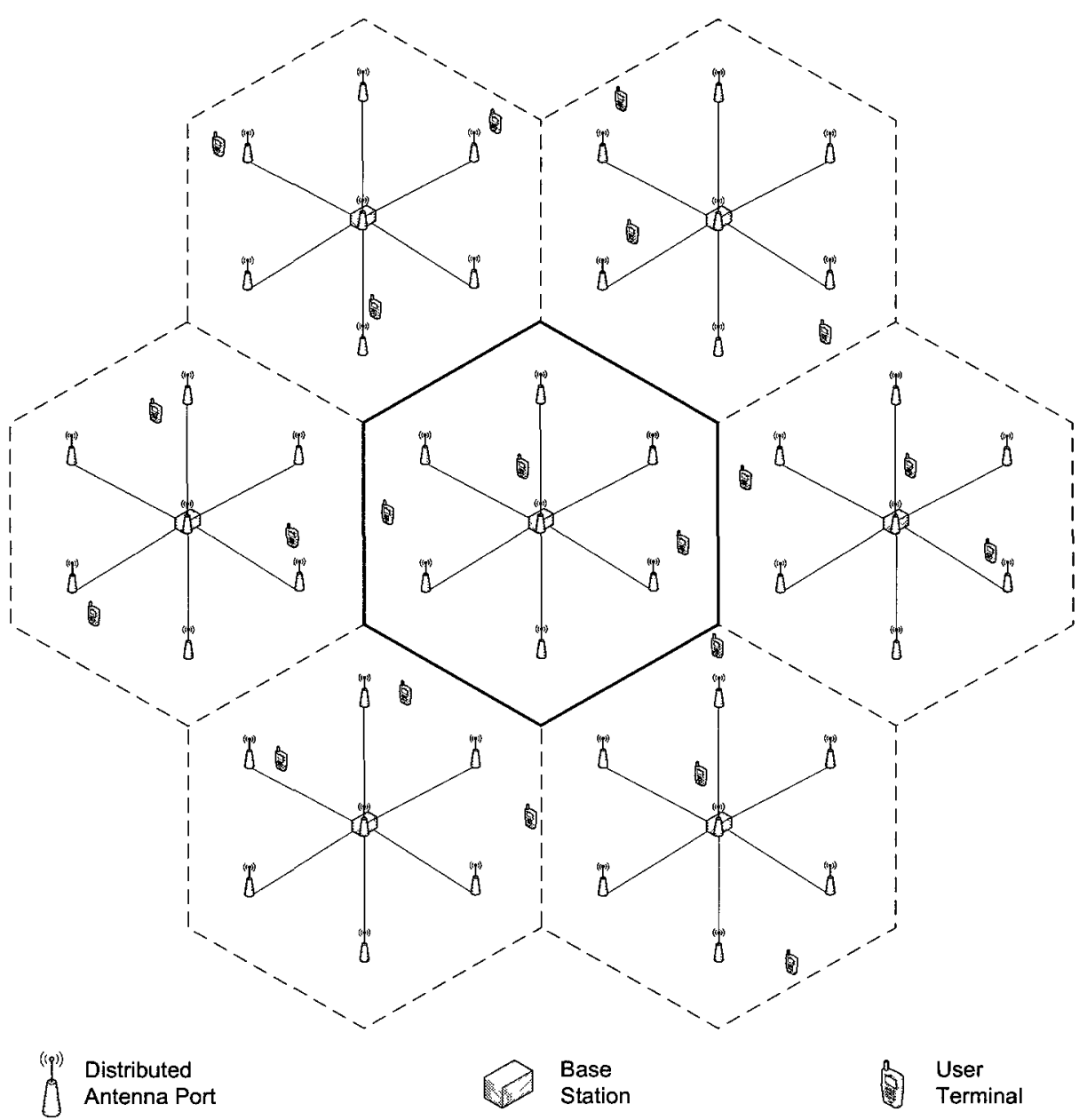

Figure 3.9: A seven-cell DAS with single-cell processing, $K=3 \mathrm{UTs}$ per RB, and $L=7$ ports per cell.

section, a multi-cell system consisting of seven hexagonal cells (i.e., $M=7$ ) is considered (see Fig. 3.9). In this system, the BSs operate independently and hence, the UTs experience inter-cell interference. All other system and simulation parameters remain the same as in Section 3.5.1. The results presented in the following examples pertain to the cell that is located at the center of this seven-cell system. In other words, a single tier of interfering cells is considered for the performance evaluation.

Example 3.7. In Fig. 3.10, a comparison analogous to the one in Fig. 3.3 is considered, where $N_{t}=2$ and $\left|\mathcal{C}_{k m}\right|=3$ for all $k, m$ and inter-cell interference is present. It can be seen from this figure that when $P_{t}$ is relatively low, the aggregate cell spectral 
efficiency achieved by both DAS schemes and by the corresponding CAS schemes increases with $P_{t}$. This is due to the fact that the system is noise-limited in this region, which is similar to the observation from Fig. 3.3. However, at higher values of $P_{t}$, the achieved spectral efficiencies demonstrate a saturation behaviour, which suggests that the system is interference-limited.

Example 3.8. In Fig. 3.11, a comparison similar to the one in Fig. 3.10 is considered, except that now $N_{t}=3$. It can be observed from Fig. 3.11 that with the addition of a single antenna at each port, the aggregate spectral efficiency achieved by DAS BD approaches that of DAS ZF-DPC.

This example is also analogous to Example 3.2, with the difference being the presence of ICI. It can be seen that the spectral efficiencies achieved by each of the DAS and CAS schemes in Example 3.2 serve as an upper bound on those achieved in this example. Furthermore, the interference-limited behaviour of the system can be observed in Fig. 3.11 at higher values of $P_{t}$.

Example 3.9. In Fig. 3.12, a comparison similar to the one in Fig. 3.10 is considered, except that now $\left|\mathcal{C}_{k m}\right|=7, \forall k, m$. The observation here is similar to that in Example 3.8; that is, the performance of DAS BD approaches that of DAS ZF-DPC with the additional spatial degrees of freedom. Also, comparing the results shown in Fig. 3.12 with those in Fig. 3.5, the interference-limited behaviour can be seen at higher values of $P_{t}$ in the multi-cell environment with ICI.

\section{Effect of the Number of Coordinating Ports per UT}

Example 3.10. In this example, the impact of the number of coordinating ports per UT on the aggregate spectral efficiency per RB achieved by DAS ZF-DPC and DAS $\mathrm{BD}$ is investigated for the cell marked by a solid boundary in the multi-cell system depicted in Fig. 3.9. The results are presented in Fig. 3.13. 


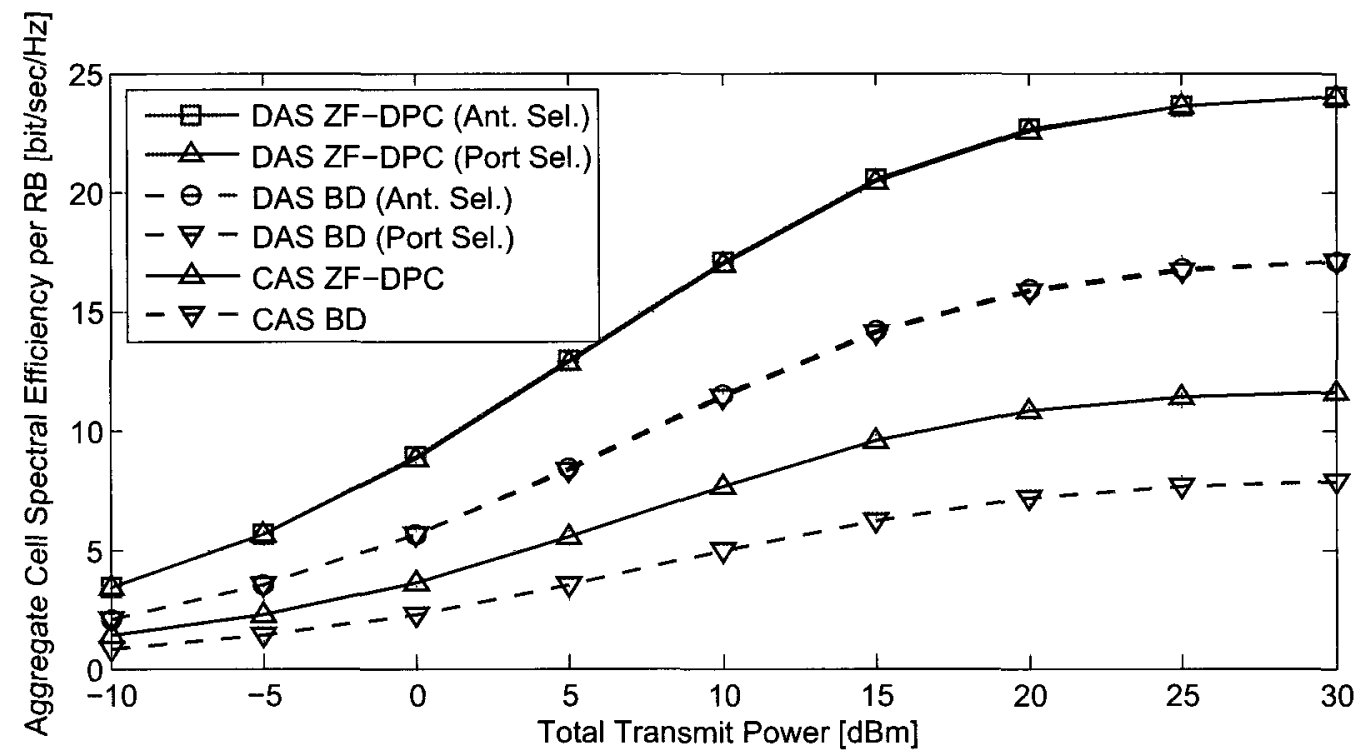

(a)

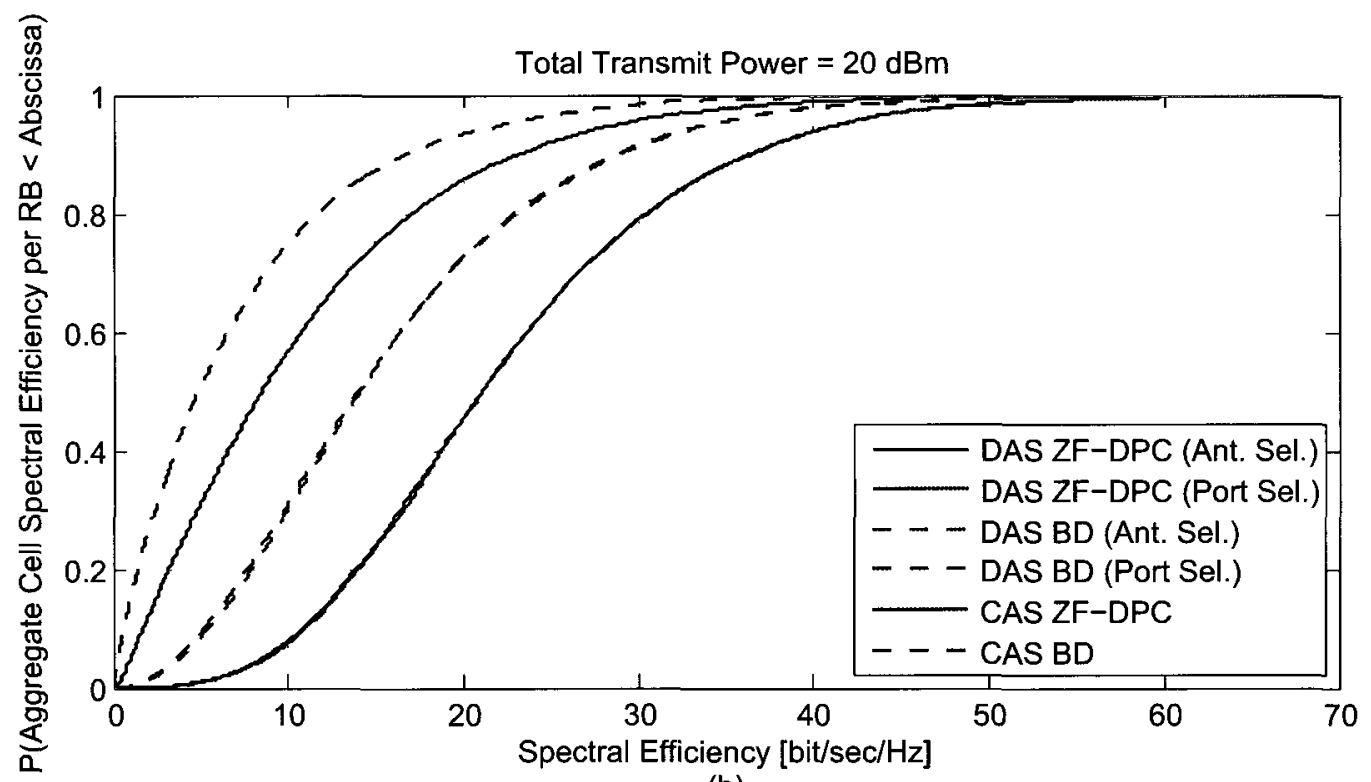

(b)

Figure 3.10: A comparison between the ergodic and outage aggregate cell spectral efficiencies per RB achieved by DAS BD and DAS ZF-DPC and those achieved by their CAS counterparts for a cell that operates in an inter-cell interference environment. $N_{t}=2$ and $\left|\mathcal{C}_{k m}\right|=3, \forall k$. 


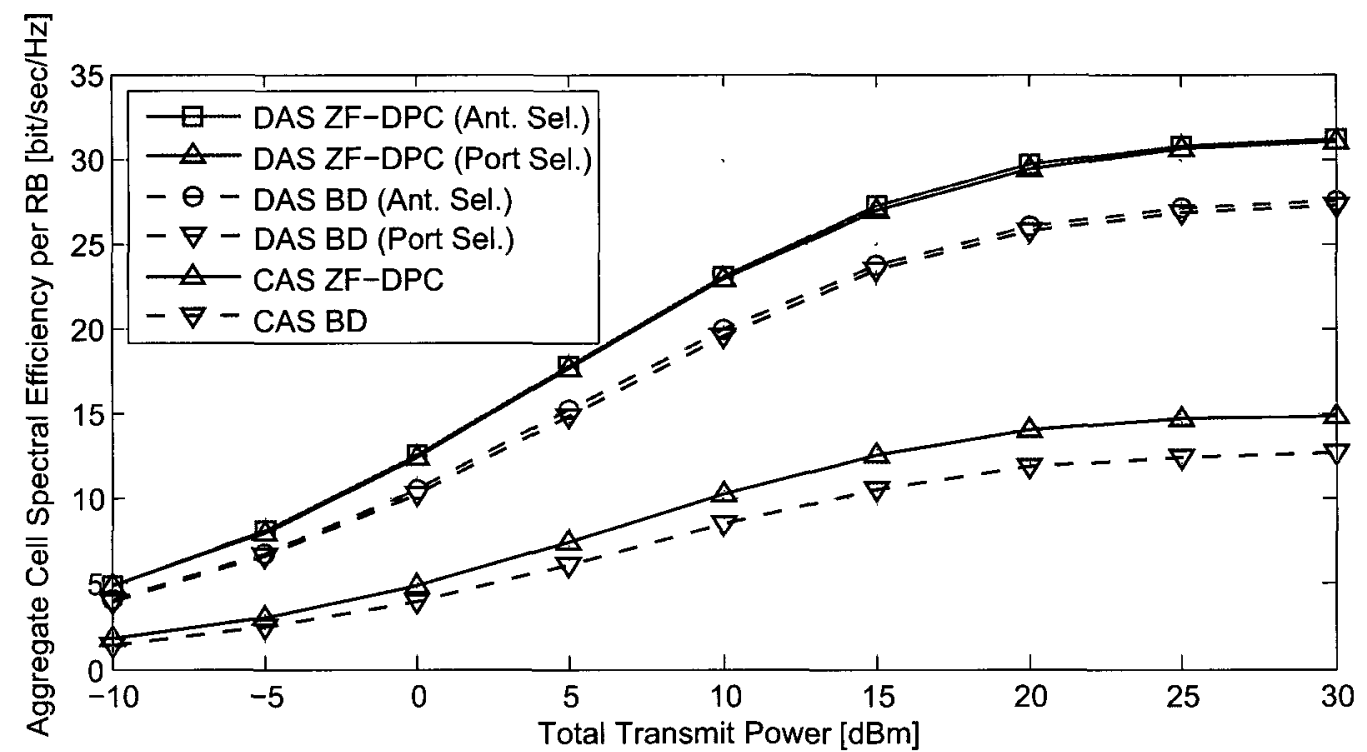

(a)

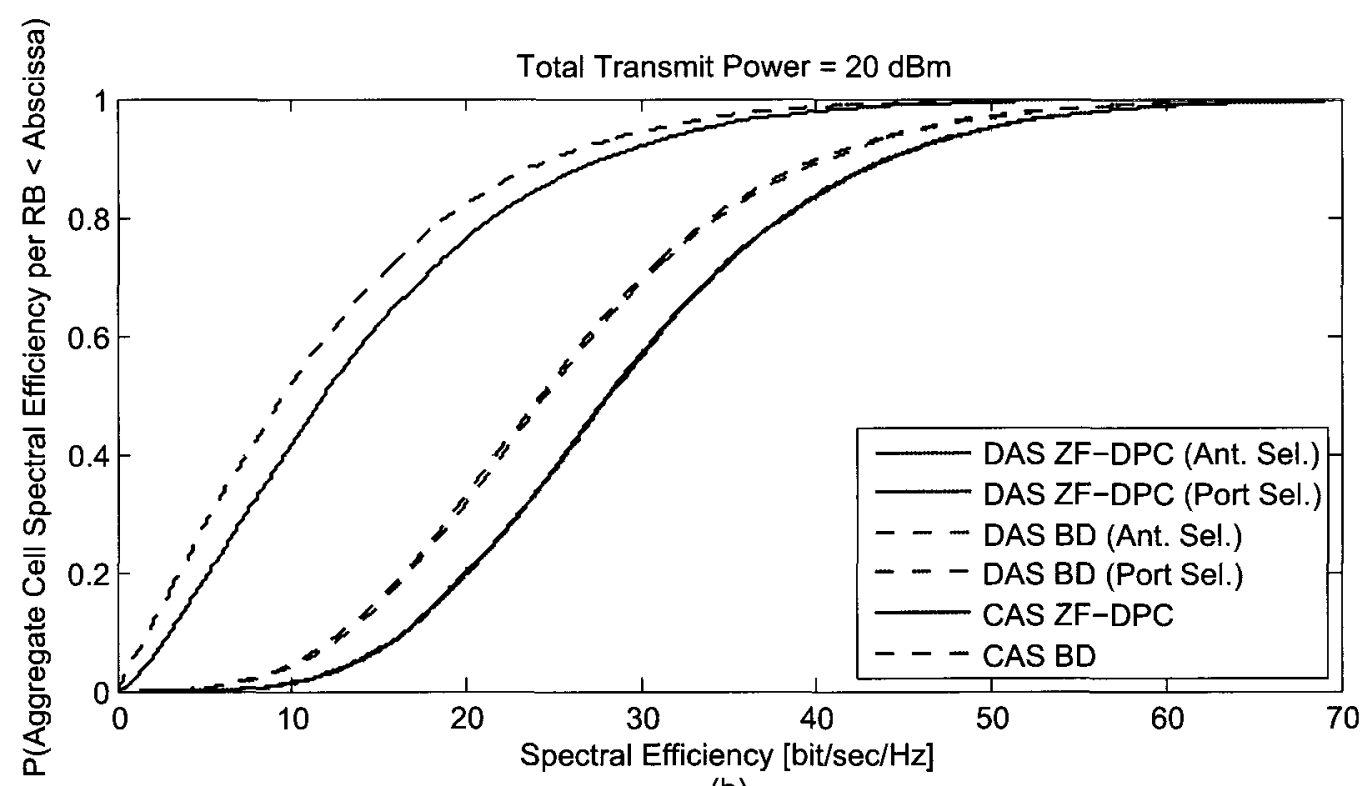

(b)

Figure 3.11: A comparison between the ergodic and outage aggregate cell spectral efficiencies per RB achieved by DAS BD and DAS ZF-DPC and those achieved by their CAS counterparts for a cell that operates in an inter-cell interference environment. $N_{t}=3$ and $\left|\mathcal{C}_{k m}\right|=3, \forall k$. 


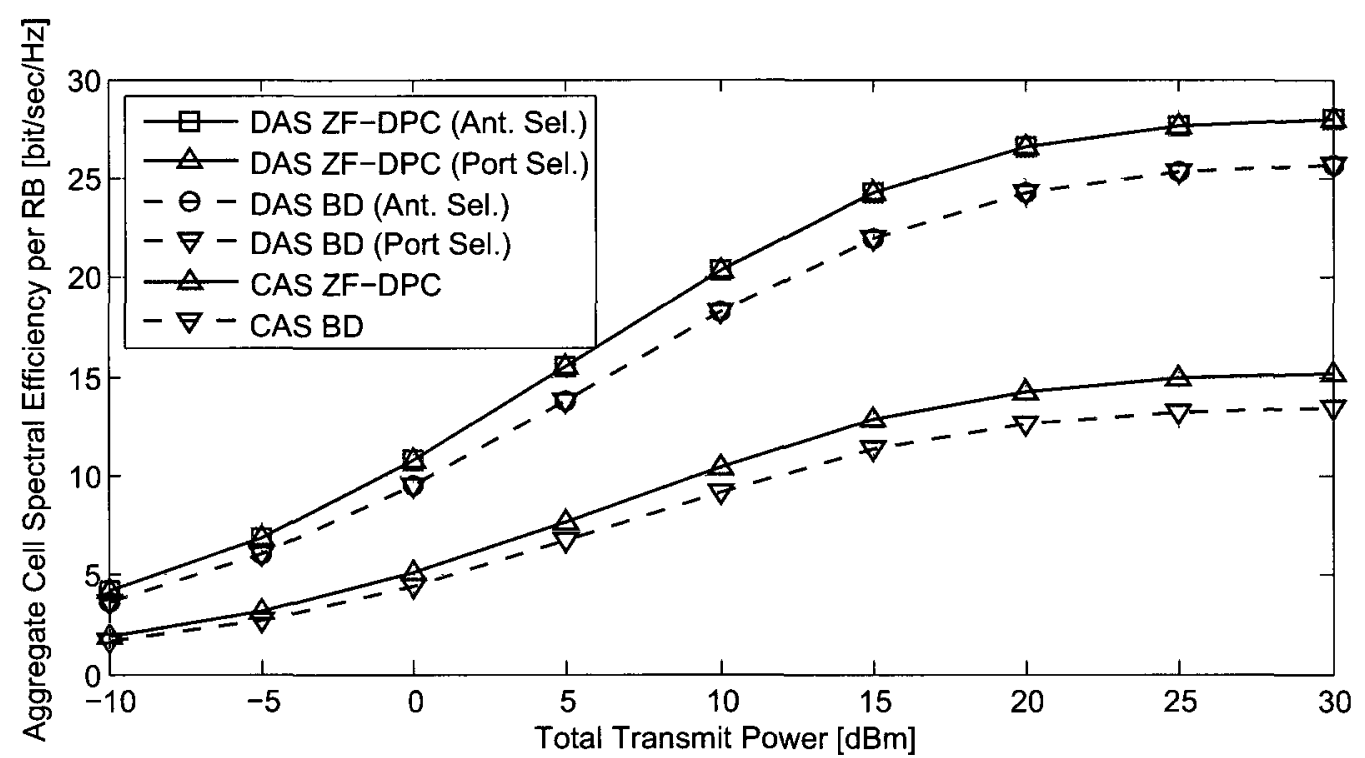

(a)

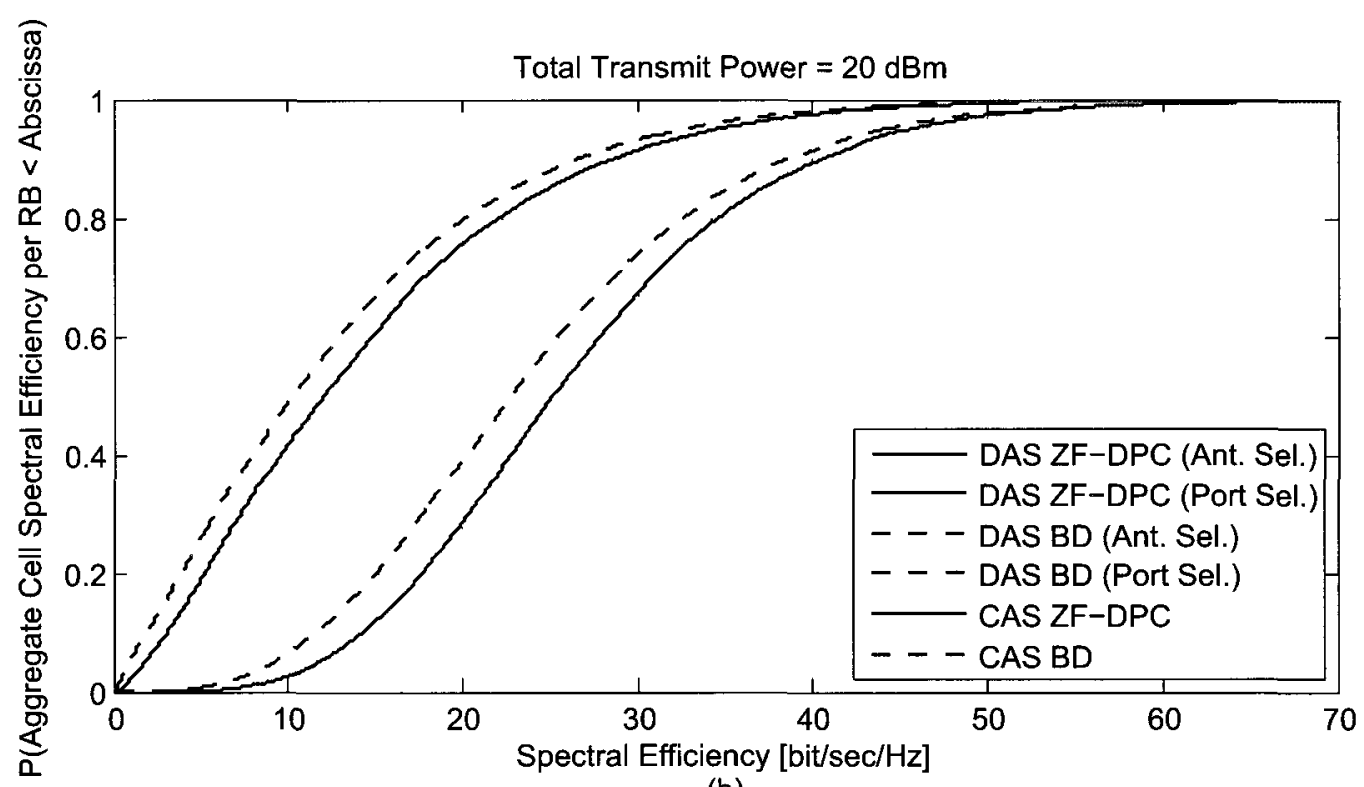

(b)

Figure 3.12: A comparison between the ergodic and outage aggregate cell spectral efficiencies per RB achieved by DAS BD and DAS ZF-DPC and those achieved by their CAS counterparts for a cell that operates in an inter-cell interference environment. $N_{t}=2$ and $\left|\mathcal{C}_{k m}\right|=7, \forall k$. 


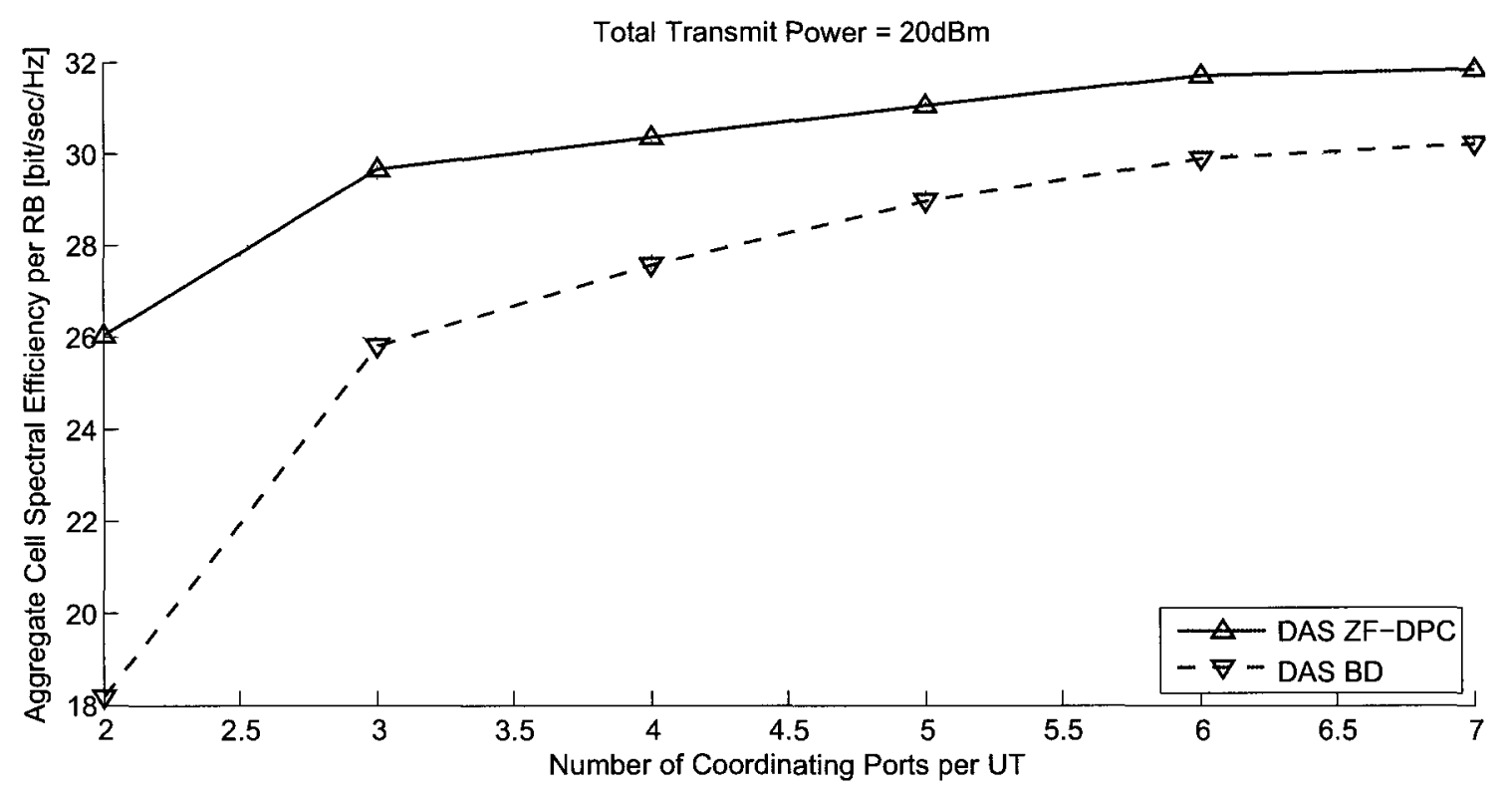

Figure 3.13: A comparison between the aggregate cell spectral efficiencies per RB achieved by DAS BD and DAS ZF-DPC for different values of $\left|\mathcal{C}_{k m}\right|$ for a cell that operates in an inter-cell interference environment. $N_{t}=3$.

The performance degrading effects of ICI can be seen by the comparing the results in this figure with those in Fig. 3.7. It can also be observed that the aggregate cell spectral efficiency increases monotonically with $\left|\mathcal{C}_{k m}\right|$ for all $k, m$. Although it may be expected that the performance would degrade as the number of active ports increases due to higher levels of interference to UTs in other cells, this is shown to not be the case. The reason for this observation is that due to the total transmit power in the cell being fixed, activating a larger number of ports only results in a redistribution the available power. Hence, the potential benefits of port selection are not fully realized in such a system with a total power constraint. In contrast, it will be shown in Chapter 4 that when the transmit power of each port is fixed, proper port selection can lead to significant performance improvement. 


\subsubsection{Multi-Cell System with Centralized Processing}

In this section, the performance of the schemes developed in Section 3.4 is assessed. Similar to Section 3.5.2, a seven-cell system is considered; however, the BSs now coordinate their transmissions in a centralized manner, and any of the $L M=49$ ports in the cluster may be selected for transmission to a particular UT (see Fig. 3.14). Additionally, the cluster of seven cells is subject to a total power constraint of $7 P_{t}$ as described in Section 3.4. To enable a comparison with the single-cell processing results in Section 3.5.2, the performance metric is chosen to be the average spectral efficiency per cell per RB. In other words, instead of considering the spectral efficiency of the cell at the center of the cluster as was previously the case, the aggregate spectral efficiency of the entire cluster is determined, and then scaled by a factor of $1 / M$.

Example 3.11. In Fig. 3.15, the average aggregate spectral efficiency per cell per RB achieved by DAS ZF-DPC and DAS BD with centralized multi-cell processing is compared with those achieved by these schemes with single-cell processing. In this system, $N_{t}=2$ and $\left|\mathcal{C}_{k}\right|=3$ for all $k$. It can be seen from this figure that DAS $\mathrm{BD}$ with multi-cell processing outperforms the corresponding scheme with single-cell processing for all values of transmit power. This gain can be attributed to

- the more flexible total power constraint for the cluster as a whole rather than one for each cell, and

- the increased flexibility in port selection (since the ports that can be selected to transmit to a particular UT need not be associated with the same cell).

However, in the case of DAS ZF-DPC, it is interesting that while centralized multicell processing outperforms single-cell processing at higher power levels, the opposite is true when the power is relatively low. This observation suggests that using the available spatial degrees of freedom for diversity is preferable instead of using them for interference mitigation through BD-based zero forcing at low power values. 


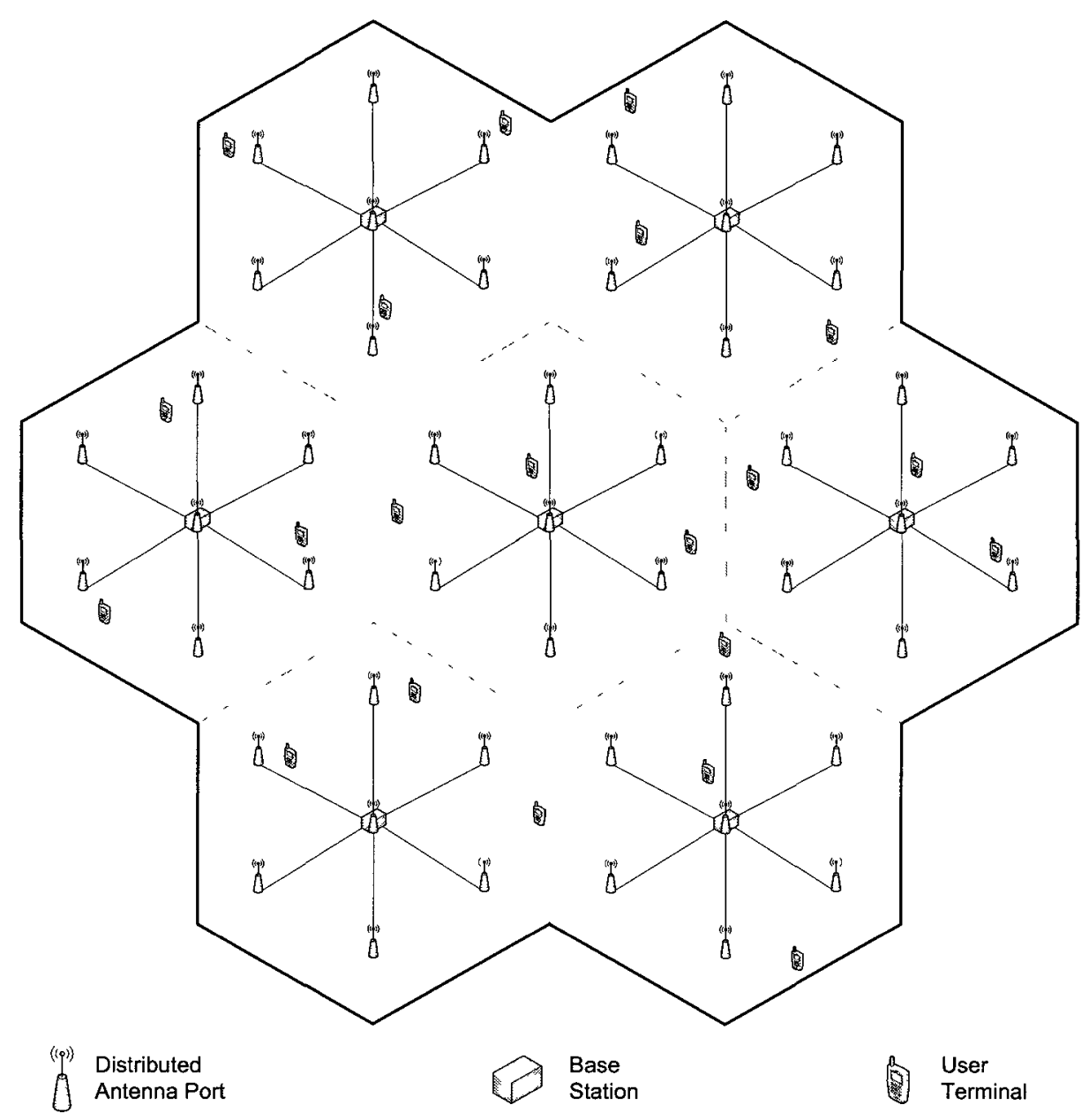

Figure 3.14: A seven-cell DAS with centralized multi-cell processing, $K=3$ UTs per $\mathrm{RB}$, and $L=7$ ports per cell.

It can also be noted by comparing the single-cell processing results for DAS ZFDPC and DAS BD in Fig. 3.15 with the corresponding ones in Fig. 3.10 that the average aggregate spectral efficiency per cell per RB achieved by these schemes is higher than the aggregate spectral efficiency per RB of the cell that is located at the center of the cluster. This difference is due to the fact that the spectral efficiencies of all the cells are considered in Fig. 3.15. Since six of the outer cells are not fully surrounded by other interfering cells, the overall spectral efficiency can be expected to be higher than that of the center cell.

Example 3.12. In this example, a comparison similar to that in Example 3.11 is 


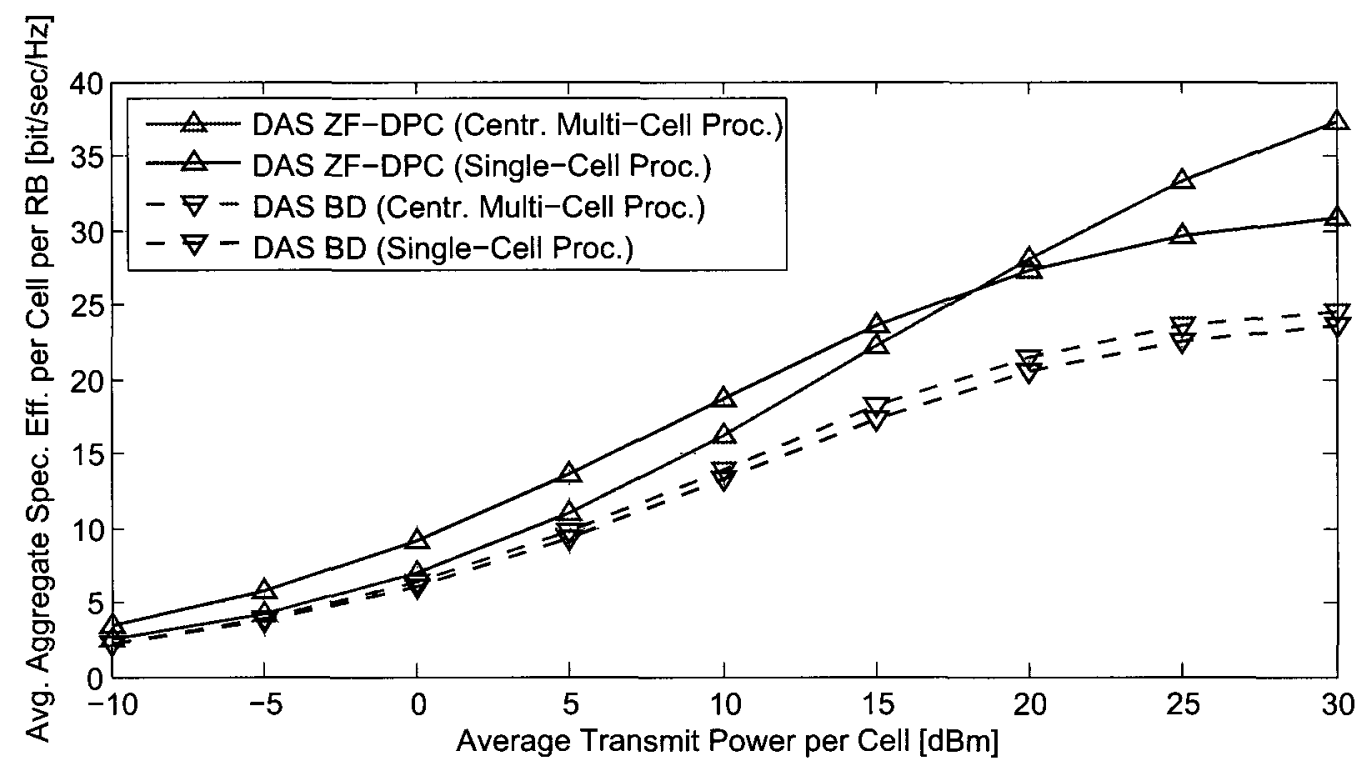

(a)

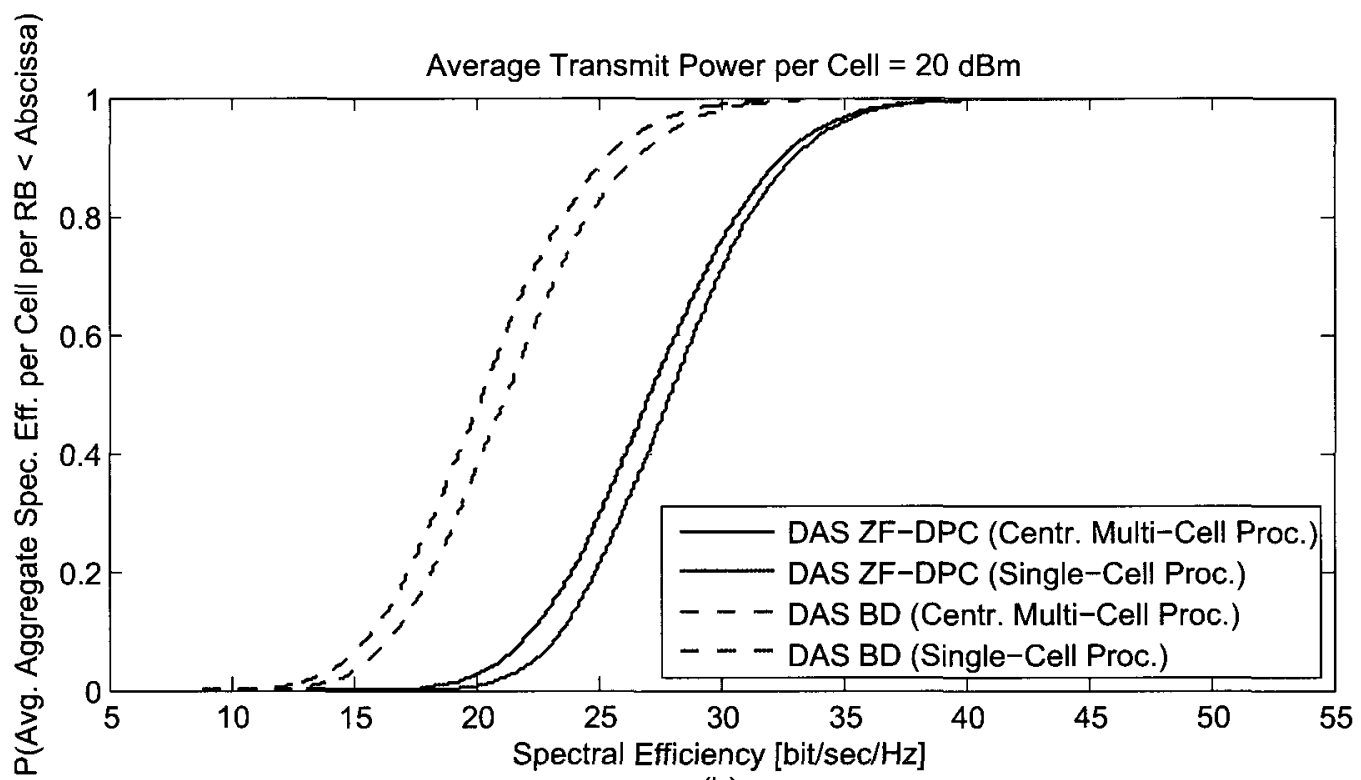

(b)

Figure 3.15: A comparison between the ergodic and outage average aggregate spectral efficiency per cell per RB achieved by DAS BD and DAS ZF-DPC with centralized multi-cell processing and with single-cell processing for a seven-cell cluster. $N_{t}=2$ and $\left|\mathcal{C}_{k}\right|=3, \forall k$. 
considered, except that $N_{t}=3$. The average aggregate spectral efficiencies per cell per RB achieved by DAS ZF-DPC and DAS BD with both single-cell and multi-cell processing for this system are shown in Fig. 3.16. The observations in this figure are similar to those in Fig. 3.15, with DAS BD with centralized multi-cell processing outperforming the corresponding scheme with single-cell processing. The DAS ZFDPC scheme with multi-cell processing can also be seen to outperform DAS ZF-DPC with single-cell processing when the transmit power levels are relatively high, and vice versa when they are relatively low. 


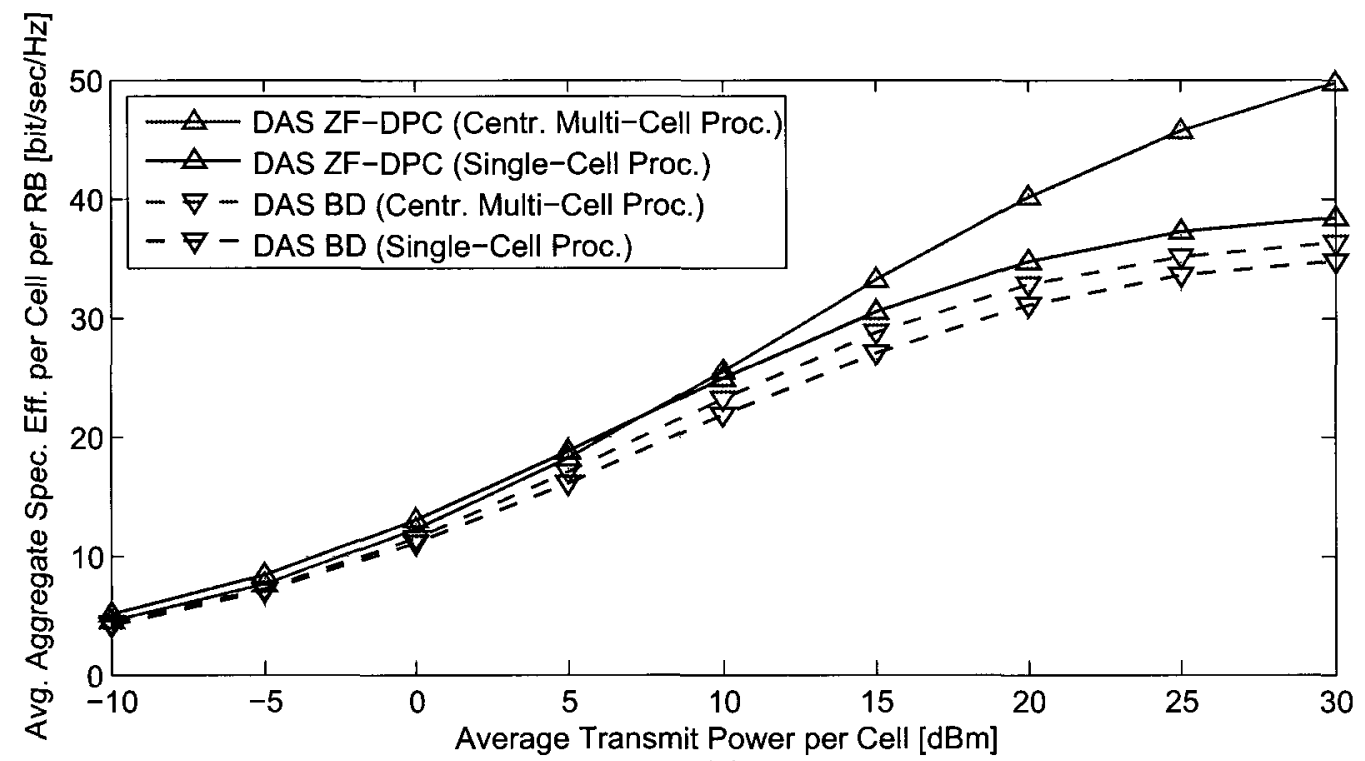

(a)

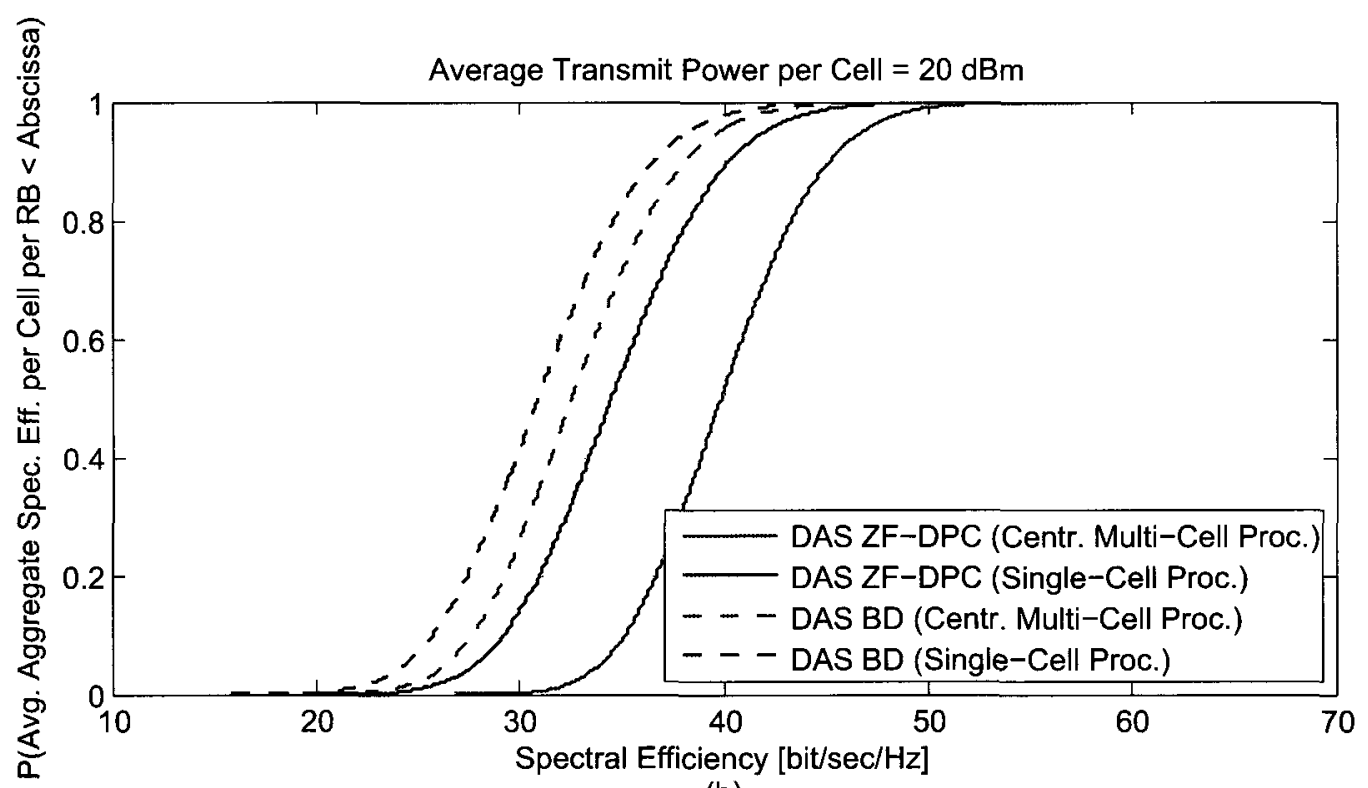

(b)

Figure 3.16: A comparison between the ergodic and outage average aggregate spectral efficiency per cell per RB achieved by DAS BD and DAS ZF-DPC with centralized multi-cell processing and with single-cell processing for a seven-cell cluster. $N_{t}=3$ and $\left|\mathcal{C}_{k}\right|=3, \forall k$. 


\section{Chapter 4}

\section{Coordinated Max-Min Fair Multi-Cell Port Selection and Beam Steering Optimization using Semidefinite Relaxation}

Recall that in each of the two coordinated downlink transmission schemes developed in Chapter 3, the subset of antennas ports that transmit to each UT is pre-selected. Although jointly selecting ports and designing the precoding coefficients would yield improved performance, this is a challenging optimization problem. In this chapter, this problem is addressed for a cellular DAS in which the UTs and ports have a single antenna each, at most a single UT is permitted to occupy a particular RB in each cell, and each port transmits using a fixed power.

\subsection{Related Literature}

Despite the envisioned benefits of using multiple antenna ports (see Section 2.1), poor selection and weighting of ports can yield low SINRs, resulting in undesirable performance. This fact has been demonstrated in [53] for cellular distributed antenna systems with no coordination among the BSs. In [54], a system with no BS coordination, similar to the one in [53], is considered. For this system the weights of the antenna ports are chosen to match the phases of the channel coefficients and either a single-port or an all-port transmission strategy is selected.

In contrast with both [53] and [54], in this work, a cluster of cells is considered 
in which the BSs organize their transmissions in a coordinated manner. Since coordination among multiple cells is a generalization of coordination within a single cell, the CoMP system considered herein subsumes those considered in [53] and [54], and offers a greater number of degrees of design freedom. Also, unlike [54], in which the transmission strategies are selected and fixed prior to choosing the antenna weights, the focus herein is on the joint optimization of the ports to be used for transmission and their corresponding weights, which are henceforth referred to as beam steering coefficients. The joint optimization of these parameters allows further exploitation of the coordination among the BSs to enable the UTs to achieve higher SINRs. However, this joint optimization problem can be shown to be NP-hard [59,60], which implies that finding the global optimal solution is computationally prohibitive for many practical systems. To circumvent this difficulty, a novel polynomial-complexity two-stage technique is proposed. This technique will later be shown to yield close-to-optimal solutions efficiently.

Before indulging into the specific problem statement and the details of the proposed two-stage technique, certain topics in optimization theory that are relevant to the formulation developed in this chapter are briefly described in the next section.

\subsection{Background: Mathematical Optimization}

In mathematics, an optrmızation problem is one in which an objective is maximized over a set of variables, such that a given set of constraints is satisfied; that is

$$
\begin{array}{cl}
\max _{\boldsymbol{x}} & f_{0}(\boldsymbol{x}), \\
\text { subject to } & f_{\imath}(\boldsymbol{x}) \leq b_{\imath}, \quad \imath=1, \ldots, m .
\end{array}
$$

In the above problem, $\boldsymbol{x}$ is the optimization varıable, $f_{0}(\boldsymbol{x})$ is the objective function, and the inequalities in (4.1b) constitute the constraint set [61, Section 1.1]. 


\subsubsection{Convex Optimization Problem}

A certain class of optimization problems are called convex optımızatıon problems. In such problems, the objective function and the functions in the constraint set are all convex. By definition, a function $f_{\imath}(\boldsymbol{x})$ is convex if the inequality

$$
f_{\imath}(\alpha \boldsymbol{x}+\beta \boldsymbol{y}) \leq \alpha f_{\imath}(\boldsymbol{x})+\beta f_{\imath}(\boldsymbol{y})
$$

is satisfied for all $\alpha, \beta \in \mathbb{R}[61$, Section 1.1].

Convex optimization problems can be solved efficiently using numerical techniques such as interior-point methods, and bear an extremely useful property: if an optimal solution exists, then it is guaranteed to be globally optimal.

\subsubsection{Semidefinite Programming}

A specific type of a convex optimization problem, known as a semıdefinıte program (SDP), is for the form

$$
\begin{aligned}
\max _{\boldsymbol{X}} & \operatorname{Tr}(\boldsymbol{C} \boldsymbol{X}), \\
\text { subject to } \quad & \operatorname{Tr}\left(\boldsymbol{D}_{\boldsymbol{i}} \boldsymbol{X}\right) \unrhd_{\imath} b_{\imath}, \quad \imath=1, \ldots, m, \\
& \boldsymbol{X} \succeq \mathbf{0},
\end{aligned}
$$

where " $\unrhd_{\imath}$ " denotes either " $\leq ", "="$, or " $\geq$ " in the $\imath$-th constraint $[61$, Section 4.6.2], [62]. In other words, the objective function is linear and the optimization variable, i.e., the matrix $\boldsymbol{X}$, is positive semidefinite (PSD).

\subsubsection{Relaxation of an Optimization Problem}

It is often the case that a particular optimization problem is difficult to solve. One candidate approach for circumventing this difficulty is to consider a relaxed version 
of this problem instead. For certain types of problems, the relaxed version is often easier to solve.

Let $\mathcal{S}$ denote the constraint set of the original optimization problem, and let $\mathcal{U}$ denote the constraint set of the corresponding relaxed problem. Using the relaxation technique, some of the constraints constituting $\mathcal{S}$ are either appropriately replaced with looser ones, or omitted altogether. Therefore, $\mathcal{S} \subset \mathcal{U}$, and the optimal value of the relaxed problem is an upper-bound on that of the original problem [63].

It is important to note that the optimal solution to the relaxed problem is not necessarily optimal, or even feasible, for the original problem. Therefore, an additional step is generally required to generate a candidate approximate solution to the original problem using the optimal solution to the relaxed problem.

\subsection{System Model}

Consider the multi-cell DAS configuration described in Section 3.3.1. In this chapter, the transmissions of the BSs in the $M$ cells are coordinated by a central controller, which is assumed to have reliable knowledge of the gains between the $L M$ ports in the $M$-cell cluster and each UT in the $M$ cells. For tractability of the optimization problem tackled herein, the ports and UTs are assumed to be equipped with one antenna each, i.e., $N_{t}=N_{r}=1$. Additionally, a multi-user system is considered in which no more than one UT within the cell is assigned the same RB; e.g., OFDMA-based systems. This is a special case of the more general scenario considered in Chapter 3 , where multiple UTs can be served simultaneously in each cell in a particular RB. Although the general case of multiple UTs per cell per RB yields a richer problem, it is also more challenging, and is not considered herein.

Each port uses a fixed power to transmit to a particular UT within the cell, which inherently causes interference to UTs that use the same RB in other cells. Restricting the antenna ports to use fixed powers facilitates their hardware design and enables 
the use of low-cost power amplifiers with relatively small dynamic ranges.

Consider a specific RB. Let $\alpha_{\ell m} \in\{0,1\}$ be the binary coefficient representing the on-off state of the $\ell$-th port in the $m$-th cell on this RB, and let $w_{\ell m}$ and $P_{\ell m}$ denote the corresponding complex beam steering coefficient and the power transmitted by this port, respectively, for $\ell=1, \ldots, L, m=1, \ldots, M$. The term beam steering coefficıent is deliberately chosen to distinguish it from general terms such as precoding coefficrent and antenna werght. This choice is motivated by the fact that, due to the fixed transmit power at each port, the corresponding coefficient has unit magnitude, i.e., it lies on the unit circle. Hence, each coefficient only steers the phase of the signals transmitted from the corresponding port.

The received signal of the UT in the $m$-th cell is given by

$y_{m}=\sum_{\ell=1}^{L} \alpha_{\ell m} \sqrt{P_{\ell m}} h_{\ell m m} w_{\ell m} x_{m}+\sum_{n=1, n \neq m}^{M} \sum_{\ell=1}^{L} \alpha_{\ell n} \sqrt{P_{\ell n}} h_{\ell n m} w_{\ell n} x_{n}+n_{m}, \quad m=1, \ldots, M$,

where $h_{\ell n m}$ is the complex-valued channel gain between the $\ell$-th port of the $n$-th cell and the UT in the $m$-th cell, and $x_{m}$ is the normalized data symbol of the UT satisfying $\mathrm{E}\left\{x_{m} x_{n}\right\}=\delta_{m n}$, where $\delta_{m n}$ is equal to 1 when $m=n$ and zero otherwise. The additive white noise of the UT in the $m$-th cell is denoted by $n_{m} \sim \mathcal{C N}\left(0, \sigma^{2}\right)$, where $\mathcal{C N}(\cdot, \cdot)$ denotes a complex Gaussian distribution.

Let $\boldsymbol{\alpha}$ be the vector containing the $L M$ port states, $\left\{\alpha_{\ell m}\right\}$, and let $\boldsymbol{w}$ be the vector containing the $L M$ beam steering coefficients, $\left\{w_{\ell m}\right\}$. The SINR of the UT in the $m$-th cell can be expressed as

$$
\operatorname{SINR}_{m}(\boldsymbol{\alpha}, \boldsymbol{w})=\frac{\left(\sum_{\ell=1}^{L} \alpha_{\ell m} \sqrt{P_{\ell m}} h_{\ell m m} w_{\ell m}\right)^{2}}{\sigma^{2}+\sum_{n=1, n \neq m}^{M}\left(\sum_{\ell=1}^{L} \alpha_{\ell n} \sqrt{P_{\ell n}} h_{\ell n m} w_{\ell n}\right)^{2}}, \quad m=1, \ldots, M
$$




\subsection{Problem Statement and Proposed Solution}

One of the key motivations of using a DAS rather than a CAS is to improve the quality of service provided to all UTs, including the cell-edge ones. Such a goal can be attained by selecting the ports and the beam steering coefficients that jointly maximize the minimum SINR. These ports and coefficients can be obtained by solving the following optimization problem:

$$
\begin{aligned}
\max _{\boldsymbol{\alpha}, \boldsymbol{w}} & \min _{m=1, \ldots, M} \operatorname{SINR}_{m}(\boldsymbol{\alpha}, \boldsymbol{w}), \\
\text { subject to } & \boldsymbol{\alpha} \in\{0,1\}^{L M}, \\
& \left|[\boldsymbol{w}]_{q}\right|=1, \quad q=1, \ldots, L M
\end{aligned}
$$

where $|\cdot|$ denotes the absolute value of the scalar argument. This problem is nonconvex and hence, difficult to solve jointly for $\boldsymbol{\alpha}$ and $\boldsymbol{w}$. In fact, for any given $\boldsymbol{\alpha}$, finding the optimal beam steering coefficients, $\boldsymbol{w}$, satisfying (4.5c) is NP-hard [60]. In a complementary fashion, for any given $\boldsymbol{w}$, finding the optimal ports state vectors, $\boldsymbol{\alpha}$, satisfying (4.5b) is also NP-hard [59]. Despite their inherent difficulty, each of these problems can be cast as an optimization problem that is amenable to techniques that efficiently yield close-to-optimal solutions. To exploit this observation, an approximate two-stage solution for (4.5) will be proposed. The first stage is described in Section 4.5, the second stage is described in Section 4.6, and the details of the two-stage approach are given in Section 4.7.

\subsection{Coordinated Multi-Cell Port Selection}

In this section, the goal is to select the set of ports that maximizes the minimum SINR observed by all UTs when the beam steering coefficients are given.

Let $\boldsymbol{w}_{0}$ be the vector of the beam steering coefficients. In [54] it was shown that, 
when the interference power is fixed, the $\boldsymbol{w}_{0}$ that maximizes the SINR is the one in which the entries are chosen to match the phases of the channel between the ports and their intended UTs; that is, the $q$-th entry of $\boldsymbol{w}_{0},\left[\boldsymbol{w}_{0}\right]_{q}$, is given by

$$
\left[\boldsymbol{w}_{0}\right]_{q} \triangleq e^{-\jmath \angle h_{\ell m m}}
$$

where $q=(m-1) M+\ell$.

From (4.4) it can be seen that the interference depends on the choice of $\boldsymbol{w}_{0}$, and hence, the aforementioned assumption of fixed interference does not necessarily hold. In other words, the choice of $\boldsymbol{w}_{0}$ in (4.6) is not necessarily optimal. Despite its potential sub-optimality, choosing the beam steering coefficients in this manner facilitates the selection of the port states. This is because each entry of $\boldsymbol{w}_{0}$ corresponds to one port and depends only on the phase of the channel gain between this port and the intended UT. Hence, if this port is chosen to be inactive (i.e., its corresponding entry of $\boldsymbol{\alpha}$ is zero), the beam steering coefficients of the active ports will not be affected.

To make the SINR expression in (4.4) more amenable to the optimization technique employed hereinafter, this expression will be rewritten using vector notation. In particular, using $\boldsymbol{w}=\boldsymbol{w}_{0}$ in (4.4), the SINR of the UT in the $m$-th cell can be expressed as

$$
\operatorname{SINR}_{m}\left(\boldsymbol{\alpha}, \boldsymbol{w}_{0}\right)=\frac{\boldsymbol{\alpha}^{T} \boldsymbol{C}_{m} \boldsymbol{\alpha}}{\sigma^{2}+\boldsymbol{\alpha}^{T} \boldsymbol{D}_{m} \boldsymbol{\alpha}} \quad m=1, \ldots, M
$$

where $C_{m} \in \mathbb{R}^{L M \times L M}$ and $D_{m} \in \mathbb{R}^{L M \times L M}$ are block-diagonal matrices defined as

$$
\boldsymbol{C}_{m}=\oplus_{\imath=1}^{m-1} \mathbf{0} \oplus \boldsymbol{B}_{m, m} \oplus_{\imath=m+1}^{M} \mathbf{0}, \quad \text { and } \quad \boldsymbol{D}_{m}=\oplus_{\imath=1}^{m-1} \boldsymbol{B}_{m, \imath} \oplus \mathbf{0} \oplus_{\imath=m+1}^{M} \boldsymbol{B}_{m, \imath}
$$

where the $\ell j$-th entry of matrix $\boldsymbol{B}_{n, m} \in \mathbb{R}^{L \times L}$ is given by

$$
\left[\boldsymbol{B}_{n, m}\right]_{\ell \jmath}=\left|h_{\ell n m} h_{\jmath n m}\right| \sqrt{P_{\ell n} P_{\jmath n}}, \quad \ell, j=1, \ldots, L, \quad n, m=1, \ldots, M
$$


A detailed description of the steps involved in obtaining the expression in (4.7) is given in Appendix A. Defining the length- $L$ vector $\boldsymbol{b}_{n, m} \triangleq\left[\begin{array}{lll}\left|h_{1 n m}\right| \sqrt{P_{1 n}} & \cdots & \left|h_{L n m}\right| \sqrt{P_{L n}}\end{array}\right]$, it can be verified that $\boldsymbol{B}_{n, m}=\boldsymbol{b}_{n, m} \boldsymbol{b}_{n, m}^{T}$. Therefore, $\boldsymbol{B}_{n, m}$ is PSD and rank-1 for all $n$ and $m$. Subsequently, $\boldsymbol{C}_{m}$ and $\boldsymbol{D}_{m}$ are PSD with ranks 1 and $M-1$, respectively, for all $m$.

Using this notation, the optimization problem corresponding to (4.5) for selecting the SINR maximizing set of ports with the given $\boldsymbol{w}_{0}$ can be cast as

$$
\begin{aligned}
\max _{\boldsymbol{\alpha}} & \min _{m=1, \ldots, M} \frac{\boldsymbol{\alpha}^{T} \boldsymbol{C}_{m} \boldsymbol{\alpha}}{\sigma^{2}+\boldsymbol{\alpha}^{T} \boldsymbol{D}_{m} \boldsymbol{\alpha}} \\
\text { subject to } & \boldsymbol{\alpha} \in\{0,1\}^{L M}
\end{aligned}
$$

This is a binary constrained optimization problem, which, using a standard polynomial transformation, can be shown to be NP-hard [59]. To find a close-to-optimal solution for this problem, the semidefinite relaxation (SDR) technique will be used [62]. To use this technique, the vector $\boldsymbol{\beta}=2 \boldsymbol{\alpha}-\mathbf{1}$ will be introduced, where $\mathbf{1} \in \mathbb{R}^{L M}$ denotes the all-one vector. Using this definition, $\boldsymbol{\beta} \in\{-1,1\}^{L M}$, and

$$
\alpha=\frac{1}{2}(\boldsymbol{\beta}+\mathbf{1})
$$

Substituting (4.9) into (4.8), the port selection problem can be formulated as

$$
\begin{aligned}
& \max _{\boldsymbol{\beta}} \min _{m=1, \ldots, M} \frac{\boldsymbol{\beta}^{T} \boldsymbol{C}_{m} \boldsymbol{\beta}+2 \boldsymbol{\beta}^{T} \boldsymbol{C}_{m} \mathbf{1}+\mathbf{1}^{T} \boldsymbol{C}_{m} \mathbf{1}}{4 \sigma^{2}+\boldsymbol{\beta}^{T} \boldsymbol{D}_{m} \boldsymbol{\beta}+2 \boldsymbol{\beta}^{T} \boldsymbol{D}_{m} \mathbf{1}+\mathbf{1}^{T} \boldsymbol{D}_{m} \mathbf{1}}, \\
& \text { subject to } \boldsymbol{\beta} \in\{-1,1\}^{L M} .
\end{aligned}
$$

To use the SDR technique, the non-homogeneous quadratic forms in the numerator and denominator in $(4.10 \mathrm{a})$ can be cast in the homogeneous forms

$$
\left[\begin{array}{ll}
\boldsymbol{\beta}^{T} & 1
\end{array}\right] \boldsymbol{E}_{m}\left[\begin{array}{ll}
\boldsymbol{\beta}^{T} & 1
\end{array}\right]^{T}, \text { and }\left[\begin{array}{ll}
\boldsymbol{\beta}^{T} & 1
\end{array}\right] \boldsymbol{F}_{m}\left[\begin{array}{ll}
\boldsymbol{\beta}^{T} & 1
\end{array}\right]^{T}
$$


respectively, where

$$
\boldsymbol{E}_{m} \triangleq\left[\begin{array}{cc}
\boldsymbol{C}_{m} & \boldsymbol{C}_{m} \mathbf{1} \\
\mathbf{1}^{T} \boldsymbol{C}_{m} & \mathbf{1}^{T} \boldsymbol{C}_{m} \mathbf{1}
\end{array}\right], \quad \text { and } \quad \boldsymbol{F}_{m} \triangleq\left[\begin{array}{cc}
\boldsymbol{D}_{m} & \boldsymbol{D}_{m} \mathbf{1} \\
\mathbf{1}^{T} \boldsymbol{D}_{m} & \mathbf{1}^{T} \boldsymbol{D}_{m} \mathbf{1}+4 \sigma^{2}
\end{array}\right]
$$

Using this form and letting $\boldsymbol{\Phi}=\boldsymbol{\beta} \boldsymbol{\beta}^{T}$, the problem in (4.10) can be written in the following form.

$$
\begin{aligned}
\max _{\boldsymbol{\Phi}, \boldsymbol{\beta}} \min _{m=1,, M} \frac{\operatorname{Tr}\left(\boldsymbol{E}_{m}\left[\begin{array}{cc}
\boldsymbol{\Phi} & \boldsymbol{\beta} \\
\boldsymbol{\beta}^{T} & 1
\end{array}\right]\right)}{\operatorname{Tr}\left(\boldsymbol{F}_{m}\left[\begin{array}{ll}
\boldsymbol{\Phi} & \boldsymbol{\beta} \\
\boldsymbol{\beta}^{T} & 1
\end{array}\right]\right)}, \\
\text { subject to } \boldsymbol{\Phi}-\boldsymbol{\beta} \boldsymbol{\beta}^{T}=\mathbf{0}, \\
\operatorname{diag}(\boldsymbol{\Phi})=\mathbf{1},
\end{aligned}
$$

where $\operatorname{diag}(\cdot)$ is the vector containing the diagonal entries of the matrix argument. In this formulation, the binary constraint in $(4.10 \mathrm{~b})$ has been replaced with the equivalent linear constraint in $(4.12 \mathrm{c})$. This equivalence can be seen by noting that when (4.12b) is satisfied, the diagonal entries of $\Phi$ will be equal to the squared entries of $\boldsymbol{\beta}$. In this case, the constraint in (4.12c) is equivalent to $\beta_{\ell m}^{2}=1, \forall \ell, m$, which is satisfied if and only if $\boldsymbol{\beta} \in\{-1,1\}^{L M}$.

\subsubsection{Positive Semidefinite Relaxation}

The constraint in (4.12b) imposes a non-convex rank-1 constraint on $\boldsymbol{\Phi}$, which results in the NP-hardness of the optimization problem in (4.12). To obtain a close-tooptimal solution of this problem, (4.12) is relaxed by replacing the equality constraint in $(4.12 \mathrm{~b})$ with a generalized matrix inequality. Doing so, the relaxed optimization 
problem corresponding to (4.12) can be expressed as:

$$
\begin{aligned}
\max _{\boldsymbol{X}, \boldsymbol{x}} \min _{m=1, \ldots, M} \frac{\operatorname{Tr}\left(\boldsymbol{E}_{m}\left[\begin{array}{ll}
\boldsymbol{X} & \boldsymbol{x} \\
\boldsymbol{x}^{T} & 1
\end{array}\right]\right)}{\operatorname{Tr}\left(\boldsymbol{F}_{m}\left[\begin{array}{ll}
\boldsymbol{X} & \boldsymbol{x} \\
\boldsymbol{x}^{T} & 1
\end{array}\right]\right)} \\
\text { subject to } \quad \boldsymbol{X}-\boldsymbol{x} \boldsymbol{x}^{T} \succeq \mathbf{0}, \\
\operatorname{diag}(\boldsymbol{X})=\mathbf{1},
\end{aligned}
$$

where $\boldsymbol{X} \in \mathbb{R}^{L M \times L M}$ and $\boldsymbol{x} \in \mathbb{R}^{L M}$ are the optimization variables corresponding to $\boldsymbol{\Phi}$ and $\boldsymbol{\beta}$ in the original problem, respectively. Neither the objective in (4.13a) nor the constraint in $(4.13 \mathrm{~b})$ is convex. To cast problem (4.13) in a more convenient form, an auxiliary variable, $t$, is introduced that lower-bounds the objective function ${ }^{1}$. Furthermore, it can be noted that the matrix $\boldsymbol{X}-\boldsymbol{x} \boldsymbol{x}^{T}$ is the Schur complement [46] of the matrix

$$
\Psi=\left[\begin{array}{ll}
\boldsymbol{X} & \boldsymbol{x} \\
\boldsymbol{x}^{T} & 1
\end{array}\right],
$$

and is PSD if and only if $\boldsymbol{\Psi}$ is PSD. Using this observation, the constraint in (4.13b) will be cast as a convex linear matrix inequality constraint in $(4.15 \mathrm{c})$ below. Now,

\footnotetext{
${ }^{1}$ The introduction of such an auxiliary variable is a relatively common practice when dealing with max-min or min-max optimization problems (see, e.g., [59]).
} 
the problem in (4.13) can be rewritten as

$$
\begin{array}{cl}
\max _{\boldsymbol{t}, \boldsymbol{X}, \boldsymbol{x}} & t, \\
\text { subject to } & t \leq \frac{\operatorname{Tr}\left(\boldsymbol{E}_{m} \boldsymbol{\Psi}\right)}{\operatorname{Tr}\left(\boldsymbol{F}_{m} \boldsymbol{\Psi}\right)}, \quad m=1, \ldots, M, \\
& \boldsymbol{\Psi}=\left[\begin{array}{ll}
\boldsymbol{X} & \boldsymbol{x} \\
\boldsymbol{x}^{T} & 1
\end{array}\right] \succeq \mathbf{0}, \\
& \operatorname{diag}(\boldsymbol{X})=\mathbf{1} .
\end{array}
$$

The problem in (4.15) is still non-convex in $(t, \boldsymbol{x}, \boldsymbol{X})$ because the inequality constraint in (4.15b) involves products of the form $t \boldsymbol{x}$ and $t \boldsymbol{X}$. Fortunately, however, it can be seen that these constraints are quasi-linear in $(t, \boldsymbol{x}, \boldsymbol{X})$. This is because both the super-level and the sub-level sets corresponding to a fixed $t$ are convex [61, Section 3.4]. The constraint in (4.15b) can be expressed in the form

$$
\operatorname{Tr}\left(\left(t \boldsymbol{F}_{m}-\boldsymbol{E}_{m}\right) \boldsymbol{\Psi}\right) \leq 0, \quad m=1, \ldots, M
$$

To find the optimal value of $t$, it is noted that the left hand side of (4.16) is monotonically increasing in $t$ for any given $\boldsymbol{x}$ and $\boldsymbol{X}$ satisfying (4.13b). To show this, it suffices to show that $\operatorname{Tr}\left(\boldsymbol{F}_{m} \boldsymbol{\Psi}\right)$ is strictly positive. This is shown in the following lemma.

Lemma 4.1. For the matrix $\boldsymbol{F}_{m}$ defined in (4.11) and the matrix $\boldsymbol{\Psi}$ defined in (4.14),

$$
\operatorname{Tr}\left(\boldsymbol{F}_{m} \boldsymbol{\Psi}\right)>0, \quad m=1, \ldots, M
$$

Proof. See Appendix A.

Using this lemma, the optimal $t$, which is denoted by $t^{\star}$, can be obtained by 
solving a series of convex feasibility problems. Each of these problems is of the form

$$
\begin{aligned}
\text { find } & \boldsymbol{X}, \boldsymbol{x} \\
\text { subject to } & \operatorname{Tr}\left(\left(t_{0} \boldsymbol{F}_{m}-\boldsymbol{E}_{m}\right) \boldsymbol{\Psi}\right) \leq 0, \quad m=1, \ldots, M, \\
& \boldsymbol{\Psi}=\left[\begin{array}{ll}
\boldsymbol{X} & \boldsymbol{x} \\
\boldsymbol{x}^{T} & 1
\end{array}\right] \succeq \mathbf{0}, \\
& \operatorname{diag}(\boldsymbol{X})=\mathbf{1} .
\end{aligned}
$$

For each instance of this problem, the value of $t_{0}$ is fixed. To determine an interval within which it lies, it is noted that at the optimal solution, $t_{0}$ represents the minimum SINR observed by the UTs. This SINR is upper-bounded by

$$
t_{\max }=\min _{m=1, \ldots, M} \frac{\mathbf{1}^{T} \boldsymbol{C}_{m} \mathbf{1}}{\sigma^{2}}
$$

which corresponds to a situation in which there is no interference and all ports are active. Hence, the optimal $t_{0}$ must lie in the interval $\left[0, t_{\max }\right]$.

Using an argument analogous to the one in [61, Section 4.2.5], it can be seen that if (4.17) is feasible for a particular value of $t_{0}$, then $t_{0} \leq t^{\star}$. Conversely, if (4.17) is infeasible for this value of $t_{0}$, then $t_{0}>t^{\star}$. Hence, the optimal value of $t_{0}$ must lie on the boundary of the feasible set of (4.15) and can be found using a bisection search.

\subsubsection{Randomization for Coordinated Port Selection}

Let $\boldsymbol{X}^{\star}$ and $\boldsymbol{x}^{\star}$ denote the optimal solution of (4.15) corresponding to $t=t^{\star}$ obtained by the bisection search. To obtain a candidate solution of the optimization problem in (4.10), the Gaussian randomization technique will be used. This technique is known to yield a close-to-optimal solution for NP-hard optimization problems with a similar underlying structure (see e.g., [64]). 
To apply this technique to the current problem, a set of $J_{1}$ length- $L M$ random vectors $\mathcal{V}=\left\{\boldsymbol{v}^{(\jmath)}\right\}_{j=1}^{J_{1}}$ is generated from the Gaussian distribution $\mathcal{N}\left(\boldsymbol{x}^{\star}, \boldsymbol{X}^{\star}-\boldsymbol{x}^{\star} \boldsymbol{x}^{\star T}\right)$. For sufficiently large $J_{1}$, the vectors in $\mathcal{V}$ provide an approximate solution to the following stochastic optimization problem:

$$
\begin{aligned}
\max _{\substack{\boldsymbol{X}^{\star}=E\left\{\boldsymbol{v} \boldsymbol{v}^{T}\right\} \\
\boldsymbol{x}^{\star}=E\{\boldsymbol{v}\}}} & t, \\
\text { subject to } & \mathrm{E}\left\{\left[\begin{array}{ll}
\boldsymbol{v}^{T} & 1
\end{array}\right]\left(t \boldsymbol{F}_{m}-\boldsymbol{E}_{m}\right)\left[\begin{array}{ll}
\boldsymbol{v}^{T} & 1
\end{array}\right]^{T}\right\} \leq 0, m=1, \ldots, M, \\
& \mathrm{E}\left\{[\boldsymbol{v}]_{r}^{2}\right\}=1, \quad r=1, \ldots, L M .
\end{aligned}
$$

Since $\boldsymbol{X}^{\star}-\boldsymbol{x}^{\star} \boldsymbol{x}^{\star T} \succeq \mathbf{0}$, it can be seen that this optimization problem is equivalent to the one in (4.15). Hence, it can be seen that the set of vectors in $\mathcal{V}$ solve the problem in (4.15) on average [62].

The goal now is to use the vectors in $\mathcal{V}$ to extract candidate solutions to the problem in (4.10). To do so, each realization of $\boldsymbol{v}^{(\jmath)} \in \mathcal{V}$ is quantized and the corresponding objective in (4.10) is evaluated. In particular, for each $\boldsymbol{v}^{(\jmath)} \in \mathcal{V}$, a candidate binary solution $\tilde{\boldsymbol{\beta}}^{(j)}$ is obtained as follows:

$$
\tilde{\boldsymbol{\beta}}^{(\jmath)}=\operatorname{sgn}\left(\boldsymbol{v}^{(\jmath)}-\boldsymbol{x}^{\star}\right), \quad \jmath=1, \ldots, J_{1},
$$

where $\operatorname{sgn}(\cdot)$ is the element-wise signum function. Using (4.9), the corresponding candidate solutions of (4.8) are obtained and the one yielding the largest objective is chosen; i.e.,

$$
\boldsymbol{\alpha}^{\star}=\arg \max _{\jmath=1,, J} \min _{m=1, M} \operatorname{SINR}_{m}\left(\tilde{\boldsymbol{\alpha}}^{(\jmath)}, \boldsymbol{w}_{0}\right) .
$$

The SDR-based randomization technique proposed herein for close-to-optimal port selection is summarized in the algorithm in Table 4.1.

In Section 4.9, it will be shown that when the beam steering coefficients are fixed, 
Table 4.1: Algorithm 1 - Generating a close-to-optimal set of port states

- Initialize $a=0, b=t_{\max }, \epsilon>0$.

- While $(b-a)>\epsilon$ :

- Set $t_{0}=(a+b) / 2$ and solve (4.17).

- If (4.17) is feasible, set $a=t_{0}$; else, set $b=t_{0}$.

- For $j=1, \ldots, J_{1}$,

- Generate $\boldsymbol{v}^{(\jmath)} \sim \mathcal{N}\left(\boldsymbol{x}^{\star}, \boldsymbol{X}^{\star}-\boldsymbol{x}^{\star} \boldsymbol{x}^{\star T}\right)$.

- Use (4.20) to obtain $\tilde{\boldsymbol{\beta}}^{(\jmath)}$, and (4.9) to obtain $\tilde{\boldsymbol{\alpha}}^{(\jmath)}$.

- Determine $\boldsymbol{\alpha}^{\star}$ using (4.21).

the above SDR technique with Gaussian randomization provides a close-to-optimal solution of the port selection problem with a relatively small $J_{1}$.

\subsection{Coordinated Beam Steering Optimization}

In the previous section, the problem of selecting the antenna ports that maximize the minimum SINR when the beam steering coefficients are fixed was considered. In this section, the complementary problem is considered in which the port state vectors are fixed and the beam steering coefficients are to be optimized.

Let $\boldsymbol{\alpha}_{0}$ be a given port state vector. Analogous to the approach used in Section 4.5, vector notation will be introduced to express the SINR in a convenient form that facilitates the optimization of the beam steering coefficients.

Define the $M^{2}$ matrices $\left\{\boldsymbol{Q}_{n, m}\right\}_{n, m=1}^{M}$ such that the $\ell j$-th entry of the $n m$-th matrix is given by

$$
\left[\boldsymbol{Q}_{n, m}\right]_{\ell \jmath}=\alpha_{\ell n} h_{\ell n m} h_{\jmath n m}^{*} \alpha_{\jmath n} \sqrt{P_{\ell n} P_{\jmath n}}, \quad \ell, j=1, \ldots, L, \quad n, m=1, \ldots, M
$$

where $(\cdot)^{*}$ denotes the complex conjugate. Furthermore, define block-diagonal matri- 
$\operatorname{ces} \boldsymbol{S}_{m} \in \mathbb{C}^{L M \times L M}$ and $\boldsymbol{T}_{m} \in \mathbb{C}^{L M \times L M}$ as

$$
\boldsymbol{S}_{m}=\oplus_{\imath=1}^{m-1} \mathbf{0} \oplus \boldsymbol{Q}_{m, m} \oplus_{\imath=m+1}^{M} \mathbf{0}, \quad \text { and } \quad \boldsymbol{T}_{m}=\oplus_{\imath=1}^{m-1} \boldsymbol{Q}_{m, \imath} \oplus \mathbf{0} \oplus_{\imath=m+1}^{M} \boldsymbol{Q}_{m, \imath}
$$

Using an argument analogous to the one used in Section 4.5, it can be shown that the matrices $\left\{\boldsymbol{Q}_{n, m}\right\}_{n, m=1}^{M}$ are PSD and rank-1, and that the matrices $\left\{\boldsymbol{S}_{m}\right\}_{m=1}^{M}$ and $\left\{\boldsymbol{T}_{m}\right\}_{m=1}^{M}$ are PSD with ranks 1 and $M-1$, respectively.

Using this notation, the SINR of the UT in the $m$-th cell can be expressed as

$$
\operatorname{SINR}_{m}\left(\boldsymbol{\alpha}_{0}, \boldsymbol{w}\right)=\frac{\boldsymbol{w}^{H} \boldsymbol{S}_{m} \boldsymbol{w}}{\sigma^{2}+\boldsymbol{w}^{H} \boldsymbol{T}_{m} \boldsymbol{w}} \quad m=1, \ldots, M
$$

With the port state vector being fixed as $\boldsymbol{\alpha}_{0}$, the general optimization problem in (4.5) reduces to

$$
\begin{gathered}
\max _{\boldsymbol{w}} \min _{m=1,, M} \frac{\boldsymbol{w}^{H} \boldsymbol{S}_{m} \boldsymbol{w}}{\sigma^{2}+\boldsymbol{w}^{H} \boldsymbol{T}_{m} \boldsymbol{w}}, \\
\text { subject to } \quad\left|[\boldsymbol{w}]_{q}\right|=1, \quad q=1, \ldots, L M,
\end{gathered}
$$

where the constraint in (4.23b) ensures that the entries of $\boldsymbol{w}$ lie on the unit circle, i.e., the power of each port is constant. This problem is non-convex, and in fact, a variant of it, in which the antennas have to satisfy a total power constraint but no unit circle constraints, has been shown to be NP-hard [60]. A close-to-optimal solution to the problem considered in [60] was obtained using the SDR-based Gaussian randomization technique. This technique was also used for the max-min fair problems considered in [65] and [66], wherein, in contrast to the above optimization problem, the antennas are also subject to a total power constraint. Following the lead of $[60,65,66]$, the SDRbased Gaussian randomization technique will be utilized to find a close-to-optimal solution for the beam steering optimization problem in (4.23) with the unit circle constraint in (4.23b). 
Similar to the approach used in Section 4.5, in applying the SDR technique, the optimization problem in (4.23) is relaxed by letting $\boldsymbol{\Upsilon}=\boldsymbol{w} \boldsymbol{w}^{H}$, and subsequently dropping the rank-1 constraint on $\Upsilon$. Let $\boldsymbol{W} \in \mathbb{C}^{L M \times L M}$ be the counterpart of $\Upsilon$ in the relaxed problem. Introducing an auxiliary variable $s$ that serves as a lowerbound on the objective function in $(4.23 \mathrm{a})$, the relaxed optimization problem can be expressed as

$$
\begin{array}{rl}
\max _{s, \boldsymbol{W}} & s, \\
\text { subject to } & s \sigma^{2}+\operatorname{Tr}\left(\left(s \boldsymbol{T}_{m}-\boldsymbol{S}_{m}\right) \boldsymbol{W}\right) \leq 0, \quad m=1, \ldots, M \\
& \operatorname{diag}(\boldsymbol{W})=\mathbf{1}, \\
& \boldsymbol{W} \succeq \mathbf{0}
\end{array}
$$

Because of the product of the optimization variables in $(4.24 \mathrm{~b})$, this constraint is not convex. However, similar to the relaxation of the port selection problem in Section 4.5, it can be shown that this problem is quasi-linear, and that its optimal solution lies on the boundary of the feasible set. This point can be found by using a bisection search over $s$. Let $s_{0}$ denote the value of $s$ at any iteration of the bisection search. We seek the maximum value of $s_{0}$ for which the following feasibility problem has a solution.

$$
\begin{aligned}
\text { find } & \boldsymbol{W} \\
\text { subject to } & s_{0} \sigma^{2}+\operatorname{Tr}\left(\left(s_{0} \boldsymbol{T}_{m}-\boldsymbol{S}_{m}\right) \boldsymbol{W}\right) \leq 0, \quad m=1, \ldots, M, \\
& \operatorname{diag}(\boldsymbol{W})=\mathbf{1} \\
& \boldsymbol{W} \succeq \mathbf{0} .
\end{aligned}
$$

It is straightforward to see that this problem is convex, and hence, can be solved efficiently for any given $s_{0}$. However, to facilitate the bisection search, it is desirable to upper-bound $s_{0}$. 
Let $s_{\max }$ denote the upper-bound on the value of $s_{0}$. A candidate $s_{\max }$ can be obtained by upper-bounding the SINR expression in (4.22). In particular, since, by construction, $\boldsymbol{T}_{m} \succeq 0$, we have

$$
\begin{aligned}
\operatorname{SINR}_{m}\left(\boldsymbol{\alpha}_{0}, \boldsymbol{w}\right) & \leq \frac{\boldsymbol{w}^{H} \boldsymbol{S}_{m} \boldsymbol{w}}{\sigma^{2}} \\
& \leq \frac{\|\boldsymbol{w}\|_{2}^{2}}{\sigma^{2}}\left\|\boldsymbol{S}_{m}\right\|_{2} \\
& \leq \frac{L M}{\sigma^{2}}\left\|\boldsymbol{S}_{m}\right\|_{2}
\end{aligned}
$$

where (4.26) follows from the submultiplicative property of the 2-norm [67], and (4.27) follows from the fact that $\|\boldsymbol{w}\|_{2}^{2} \leq L M$. Hence, a candidate value of $s_{\max }$ is

$$
s_{\max }=\min _{m=1, \ldots, M} \frac{L M\left\|\boldsymbol{S}_{m}\right\|_{2}}{\sigma^{2}} .
$$

Let $\boldsymbol{W}^{\star}$ denote the solution of the relaxed problem corresponding to the optimal $s_{0}$ obtained from the bisection search. The Gaussian randomization technique is then used to generate an approximate solution of the original problem in (4.23). To apply this technique, a set of $J_{2}$ length- $L M$ random vectors, $\mathcal{Z}=\left\{z^{(\jmath)}\right\}_{\jmath=1}^{J_{2}}$, is drawn from the Gaussian distribution $\mathcal{C N}\left(\mathbf{0}, \boldsymbol{W}^{\star}\right)$. Similar to the discussion in Section 4.5.2, for sufficiently large $J_{2}$, these vectors provide an approximate solution to the following stochastic optimization problem:

$$
\begin{array}{ll}
\max _{\boldsymbol{W}^{\star}=\mathrm{E}\left\{\boldsymbol{z} \boldsymbol{z}^{H}\right\}} & s, \\
\text { subject to } & \mathrm{E}\left\{s \sigma^{2}+\boldsymbol{z}^{H}\left(s \boldsymbol{T}_{m}-\boldsymbol{S}_{m}\right) \boldsymbol{z}\right\} \leq 0, m=1, \ldots, M, \\
& \mathrm{E}\left\{\left|[\boldsymbol{z}]_{q}\right|\right\}=1, \quad q=1, \ldots, L M .
\end{array}
$$

In other words, the set of vectors in $\mathcal{Z}$ solve the problem in (4.24) on average [62].

Now, to obtain candidate beam steering coefficients, $\left\{\left[\tilde{\boldsymbol{w}}^{(j)}\right]_{q}\right\}_{q=1}^{L M}$, that lie on the 
Table 4.2: Algorithm 2-Generating close-to-optimal beam steering coefficients

- Initialize $a=0, b=s_{\max }, \epsilon>0$

- While $(b-a)>\epsilon$ :

- Set $s_{0}=(a+b) / 2$ and solve (4.25).

- If (4.25) is feasible, set $a=s_{0} ;$ else, set $b=s_{0}$.

- For $\jmath=1, \ldots, J_{2}$

- Generate $\boldsymbol{z}^{(\jmath)} \sim \mathcal{C N}\left(\mathbf{0}, \boldsymbol{W}^{\star}\right)$.

- Use (4.29) to obtain $\tilde{\boldsymbol{w}}^{(\jmath)}$.

- Determine $\boldsymbol{w}^{\star}$ using (4.30).

unit circle, the entries of each realization of $\boldsymbol{z}^{(\jmath)} \in \mathcal{Z}$ are normalized; i.e.,

$$
\left[\tilde{\boldsymbol{w}}^{(\jmath)}\right]_{q}=\frac{\left[\boldsymbol{z}^{(\jmath)}\right]_{q}}{\left|\left[\boldsymbol{z}^{(\jmath)}\right]_{q}\right|}, \quad q=1, \ldots, L M
$$

The close-to-optimal solution generated by the Gaussian randomization is the one that yields the largest minimum SINR; i.e.,

$$
\boldsymbol{w}^{\star}=\arg \max _{\jmath=1,, J_{2}} \min _{m=1,, M} \operatorname{SINR}_{m}\left(\boldsymbol{\alpha}_{0}, \tilde{\boldsymbol{w}}^{(\jmath)}\right)
$$

Using an approach analogous to the one in [66], it can be shown that, for a given $\boldsymbol{\alpha}_{0}$, the above technique yields a close-to-optimal solution to the problem in (4.23).

The beam steering optimization technique proposed in this section is summarized in the algorithm in Table 4.2 . 


\subsection{A Two-Stage Approach to obtain an Approximate Solu- tion to the Joint Optimization Problem}

In this section the techniques developed in Sections 4.5 and 4.6 are used to develop a two-stage approach for generating an efficiently-computable approximate solution to the joint optimization problem in (4.5).

In the first stage, the beam steering coefficients are chosen as in (4.6), the matrices $\left\{\boldsymbol{E}_{m}, \boldsymbol{F}_{m}\right\}_{m=1}^{M}$ are constructed, and a set of $J_{1}$ port state vectors are generated using (4.20) and (4.9). Out of those $J_{1}$ vectors, the $\hat{J} \leq J_{1}$ candidates that yield the largest minimum SINR are selected. Notice that the close-to-optimal solution of (4.8) generated by (4.21) corresponds to setting $\hat{J}=1$ and does not necessarily yield a close-to-optimal solution of the problem in (4.5), as will be shown in Section 4.9 .

In the second stage, each of the $\hat{J}$ candidate vectors obtained in the first stage is used to construct the matrices $\left\{\boldsymbol{S}_{m}, \boldsymbol{T}_{m}\right\}_{m=1}^{M}$ and the corresponding close-to-optimal beam steering coefficients are generated using (4.30). Finally, the approximate solution of problem (4.5) is chosen to be the pair of port state vector and beam steering coefficient vector that jointly yield the largest objective in (4.5a).

It will be shown in Section 4.9 that this two-stage approach, with relatively small

$J_{1}$ and $\hat{J}$, yields a significantly better performance than that achieved by port selection with the initial beam steering coefficients, and that achieved by the close-to-optimal port state vector chosen in the first stage with the corresponding close-to-optimal beam steering coefficients obtained in the second stage.

\subsection{Complexity Analysis}

In this section, bounds will be provided on the computational complexity of the proposed techniques. More specifically, it will be shown that each of the techniques that 
yield close-to-optimal solutions in Sections 4.5 and 4.6 has a polynomial complexity. Hence, the two-stage approach proposed in Section 4.7 for obtaining an approximate solution to the joint optimization problem in (4.5) also has polynomial complexity.

\subsubsection{Computational Complexity of the First Stage}

In the first stage of the approach proposed in Section 4.7, the beam steering coefficients are fixed. In this case, the optimal port state vector could be found by exhaustive search over all possible vectors. The computational complexity of this approach is $O\left(2^{L M}\right)$, and hence, it is inefficient for large $L$ and $M$.

In contrast, the SDR-based Gaussian randomization technique proposed in Section 4.5 involves solving a sequence of convex optimization problems, each with a PSD constraint (refer to (4.17)). For general-use solvers, e.g., CVX [68], the complexity of solving problems of this form would be $O\left((L M)^{6.5} \log \left(1 / \epsilon_{0}\right)\right)$, where $\epsilon_{0}>0$ is the solution accuracy [69]. However, the problem in (4.17) has a particular structure that can exploited to develop more efficient solving techniques. For instance, the primaldual path-following interior-point method developed in [70] has been particularized in [62] to solve a PSD-constrained convex optimization problem similar to the one in (4.17) with complexity $O\left((L M)^{4.5} \log \left(1 / \epsilon_{0}\right)\right)$.

Let $\epsilon_{1}>0$ be the solution accuracy of the bisection search used in Section 4.5. Since this search is over the interval $\left[0, t_{\max }\right]$ and its convergence rate is exponential, the number of bisection search iterations is given by $\log \left(t_{\max } / \epsilon_{1}\right)$, where $t_{\max }$ is defined in (4.18). For the Gaussian randomization procedure described in Section 4.5.2, the computational complexity of generating and evaluating the objective corresponding to the $J_{1}$ random samples is $O\left((L M)^{2} J_{1}\right)$ [63]. Now, combining these observations, it can be seen that the complexity of the proposed port selection technique is

$$
O\left((L M)^{4.5} \log \left(1 / \epsilon_{0}\right) \log \left(t_{\max } / \epsilon_{1}\right)+(L M)^{2} J_{1}\right)
$$




\subsubsection{Computational Complexity of the Second Stage}

In the second stage, close-to-optimal beam steering coefficients for a given port state vector could be obtained by discretizing each coefficient using $N_{b}$ bins. Then, the coefficients that yield the largest minimum SINR could be determined by using an exhaustive search over all possible bin combinations. The complexity of such as ap-

proach would be $O\left(N_{b}^{L M}\right)$, which, similar to the exhaustive search in the port selection problem, is computationally inefficient. However, in Section 4.6, close-to-optimal beam steering coefficients are generated using a variation of the SDR-based Gaussian randomization technique. Using a discussion analogous to the one in Section 4.8.1, it can be shown that the complexity of this technique is

$$
O\left((L M)^{4.5} \log \left(1 / \epsilon_{0}\right) \log \left(s_{\max } / \epsilon_{1}\right)+(L M)^{2} J_{2}\right)
$$

\subsubsection{Computational Complexity of the Two-Stage Approach}

Using exhaustive search to solve the joint optimization problem in (4.5) with discretized beam steering coefficients involves a complexity of $O\left(\left(2 N_{b}\right)^{L M}\right)$. This complexity is computationally prohibitive, even for a relatively small system. For example, for a two-cell cluster with seven ports in each cell, and $N_{b}=100$, the exhaustive search involves about $200^{14} \approx 1.64 \times 10^{32}$ combinations.

Using the two-stage approach presented in Section 4.7 and the complexity discussions in Sections 4.8.1 and 4.8.2, it can be readily seen that the complexity of this technique is bounded by

$$
O\left((L M)^{4.5} \log \left(1 / \epsilon_{0}\right)\left(\hat{J} \log \left(s_{\max } / \epsilon_{1}\right)+\log \left(t_{\max } / \epsilon_{1}\right)\right)+(L M)^{2}\left(J_{2} \hat{J}+J_{1}\right)\right)
$$

The results presented in this section are summarized in Table 4.3 
Table 4.3: Complexity of the proposed techniques and the corresponding exhaustive search

\begin{tabular}{|l|l|l|}
\hline \multicolumn{2}{|l|}{ Technique } & Complexity \\
\hline Port selection (first stage) & $\begin{array}{l}\text { Exhaustıve } \\
\text { Proposed }\end{array}$ & $\begin{array}{l}O\left(2^{L M}\right) \\
O\left((L M)^{45} \log \left(\frac{1}{\epsilon_{0}}\right) \log \left(\frac{t_{\max }}{\epsilon_{1}}\right)+(L M)^{2} J_{1}\right)\end{array}$ \\
\hline $\begin{array}{l}\text { Beam steerıng optımization } \\
\text { (second stage) }\end{array}$ & $\begin{array}{l}\text { Exhaustive } \\
\text { Proposed }\end{array}$ & $\begin{array}{l}O\left(N_{b}^{L M}\right) \\
O\left((L M)^{45} \log \left(\frac{1}{\epsilon_{0}}\right) \log \left(\frac{s_{\max }}{\epsilon_{1}}\right)+(L M)^{2} J_{2}\right)\end{array}$ \\
\hline $\begin{array}{l}\text { Port selection and beam steerıng } \\
\text { optımization (two-stage approach) }\end{array}$ & Exhaustive & $O\left(\left(2 N_{b}\right)^{L M}\right)$ \\
Proposed & $O\left((L M)^{45} \log \left(\frac{1}{\epsilon_{0}}\right)\left(\hat{J} \log \left(\frac{s_{\max }}{\epsilon_{1}}\right)+\log \left(\frac{t_{\max }}{\epsilon_{1}}\right)\right)+(L M)^{2}\left(J_{2} \hat{J}+J_{1}\right)\right)$ \\
\hline
\end{tabular}

Table 4.4: System parameters used to simulate the cellular DAS architecture

\begin{tabular}{|l|c|}
\hline Parameter & Value \\
\hline UTs per cell per RB, $K$ & 1 \\
Antennas at each UT, $N_{r}$ & 1 \\
Ports per cell, $L$ & 7 \\
Antennas at each port, $N_{t}$ & 1 \\
Noise power, $\sigma^{2}$ & $-114 \mathrm{dBm}$ \\
\hline
\end{tabular}

\subsection{Performance Evaluation}

In this section, Monte Carlo simulation is used to assess the performance of the port selection and the beam steering optimization techniques presented in Sections 4.5 and 4.6, respectively, and the performance of the two-stage approach presented in Section 4.7. The numerical results reported herein are generated using MATLAB and the software package CVX [68]. The system and simulation parameters are summarized in Tables 4.4 and 4.5 .

The cellular system used for the simulation is similar to that described in Section 3.3.1. In particular, it consists of $M$ hexagonal cells, each with circumradius $r_{c}$. The BS in each cell is connected to seven ports (i.e., $L=7$ ), six of which are located uniformly at a distance of $\frac{2}{3} r_{c}$ from the center of the cell, while the seventh one is co-located with the BS. Such a system is illustrated in Fig. 4.1, where $M=7$. All ports transmit at a fixed power $P$, i.e., $P_{\ell n}=P, \forall \ell, n[53]$. In each iteration, the UTs 
Table 4.5: IMT-Advanced scenario parameters used to simulate the cellular DAS

\begin{tabular}{|l|c|c|}
\hline \multirow{2}{*}{ Parameter } & \multicolumn{2}{|c|}{ Value } \\
& Suburban Macro-Cell & Urban Macro-Cell \\
\hline Inter-BS distance & $1299 \mathrm{~m}$ & $500 \mathrm{~m}$ \\
Elevation of each port & $15 \mathrm{~m}$ & $15 \mathrm{~m}$ \\
Elevation of each UT & $1.5 \mathrm{~m}$ & $1.5 \mathrm{~m}$ \\
Carrier frequency & $2 \mathrm{GHz}$ & $2 \mathrm{GHz}$ \\
Path loss and shadowing & $\mathrm{NLOS}[57]$ & NLoS [57] \\
Log-normal shadowing std. dev., $\sigma_{s}$ & $8 \mathrm{~dB}$ & $6 \mathrm{~dB}$ \\
\hline
\end{tabular}

are dropped randomly in each cell.

Similar to Section 3.3.1, a standard communication channel model is considered with quasi-static frequency-flat Rayleigh fading, log-normal shadowing and path loss components. Each complex channel gain can be expressed as $h_{\ell n m}=\sqrt{\rho\left(d_{\ell n m}\right) s_{\ell n m}} h_{\ell n m}^{\prime}$, where where $\rho(\cdot)$ is a path loss function, which depends on the propagation environment, and $d_{\ell n m}$ is the distance between the $\ell$-th port of the $n$-th cell and the UT in the $m$-th cell. Shadowing is represented by $s_{\ell n m}$, which is log-normal distributed with $0 \mathrm{~dB}$ mean and standard deviation $\sigma_{s}$ in $\mathrm{dB}$, and multipath fading is represented by $h_{\ell n m}^{\prime}$, which is complex Gaussian distributed with zero mean and unit variance.

The simulation parameters and system layout employed herein are similar to those used in Chapter 3, with a few exceptions; e.g., the UTs and ports have one antenna each. In addition to the SMa scenario considered in Chapter 3, the urban macrocell (UMa) IMT-Advanced scenario is also considered in this Chapter [57, Sections 8.4.2, A-1.3.1]. The parameters corresponding to the two scenarios are as follows (the ones for the SMa scenario are repeated here for completeness). For the SMa scenario, the distance between the BSs is $1299 \mathrm{~m}$, and for the UMa scenario, this distance is $500 \mathrm{~m}$. The corresponding shadowing standard deviation, $\sigma_{s}$, is $8 \mathrm{~dB}$ and $6 \mathrm{~dB}$, respectively. For both scenarios, the noise power, $\sigma^{2}$, is chosen to be $-114 \mathrm{dBm}[57,58]$ and the channel model is the same as that described in Section 3.5 


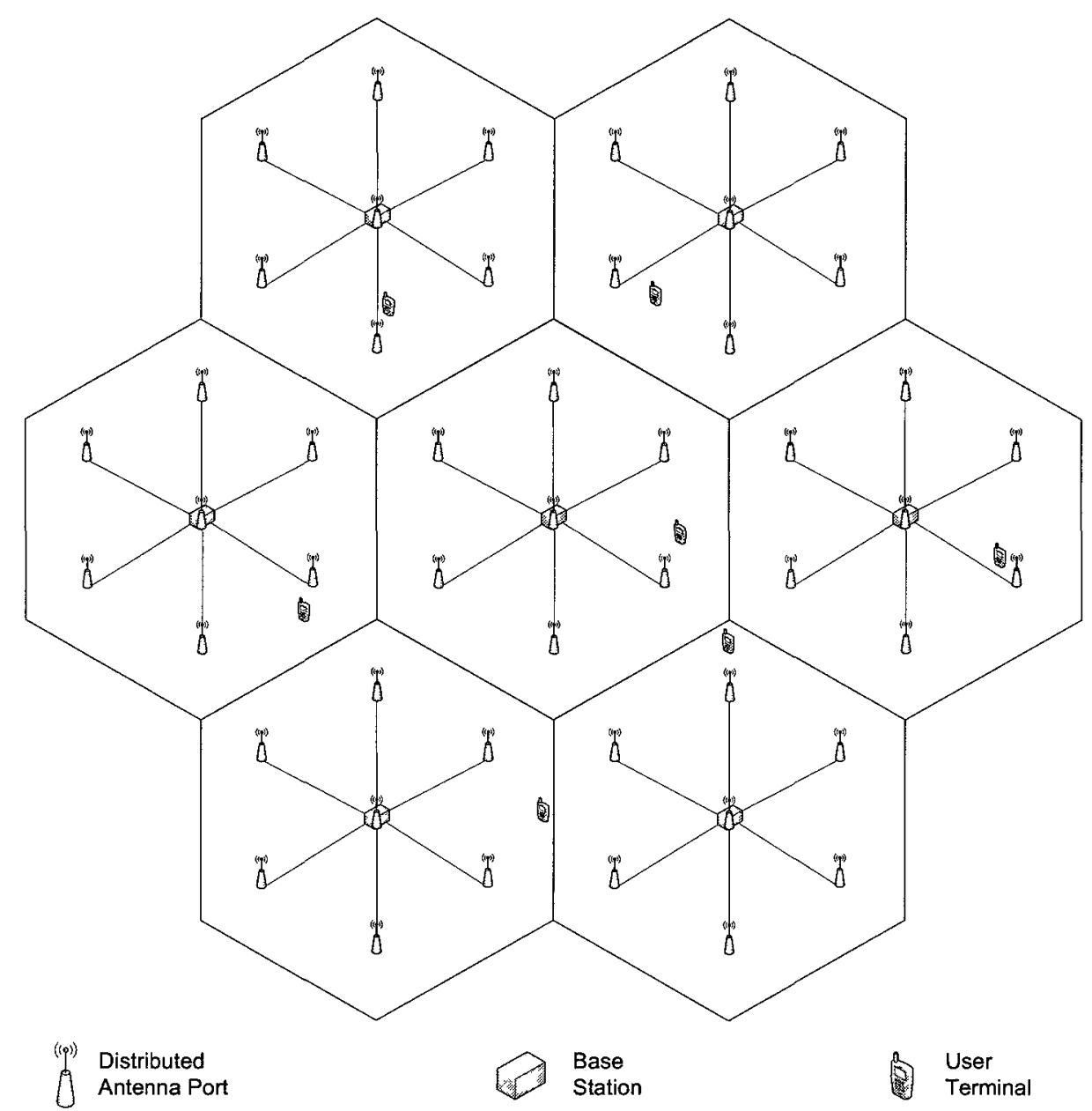

Figure 4.1: A seven-cell DAS cluster with seven ports per cell.

with the following path loss function:

$$
\rho\left(d_{\ell n m}\right)=10^{1.866+4.032 \log _{10}\left(d_{\ell n m}\right)}
$$

Example 4.1. In this example, the SMa scenario is considered. The largest minimum SINR achieved by the proposed port selection technique (summarized in Table 4.1) is compared with that of the exhaustive search described in Section 4.8. In addition, it is also compared with the largest minimum SINR achieved by other baseline transmission strategies, namely transmission from a single port and from all ports in each cell, which were investigated in [54]. To facilitate exhaustive search, the case of a two-cell 


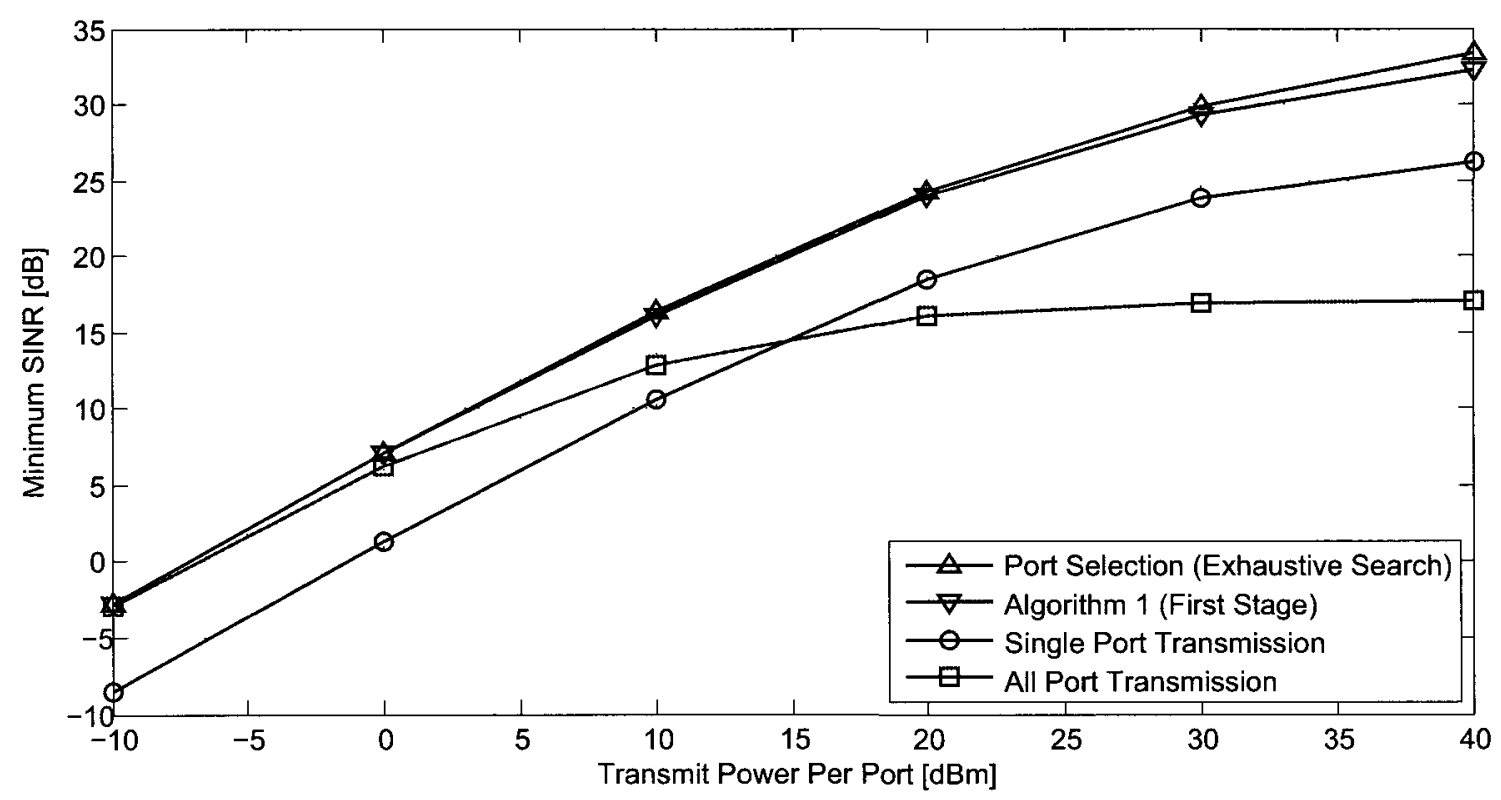

Figure 4.2: A comparison between the largest minimum SINR achieved by port selection with exhaustive search and with the technique proposed in the first stage, and that achieved by the one-port (without coordination) and all-port strategies for a two-cell cluster in the SMa scenario.

cluster (i.e., $M=2$ ) is considered, which requires searching over $2^{14} \approx 1.64 \times 10^{4}$ port state vectors. The resulting SINRs are averaged over 500 independent channel realizations. For each realization, the number of the Gaussian samples, $J_{1}$, is chosen to be 100 . The port that is chosen in each cell in the case of single-port transmission is the one with the largest channel gain to the UT in that cell.

The largest minimum SINR that is achieved by each of the techniques described above is shown in Fig. 4.2, and the corresponding spectral efficiency is shown in Fig. 4.3. From the figures, it can be seen that the performance of the proposed port selection technique approaches that of the optimal solution and is better than the two baseline strategies for the entire range of $P$.

Example 4.2. In this example, the SMa scenario is also considered, but in a cluster of $M=7$ cells. In Fig. 4.4, the largest minimum SINR achieved by the port selection technique is compared with those achieved by the single-port and all-port 


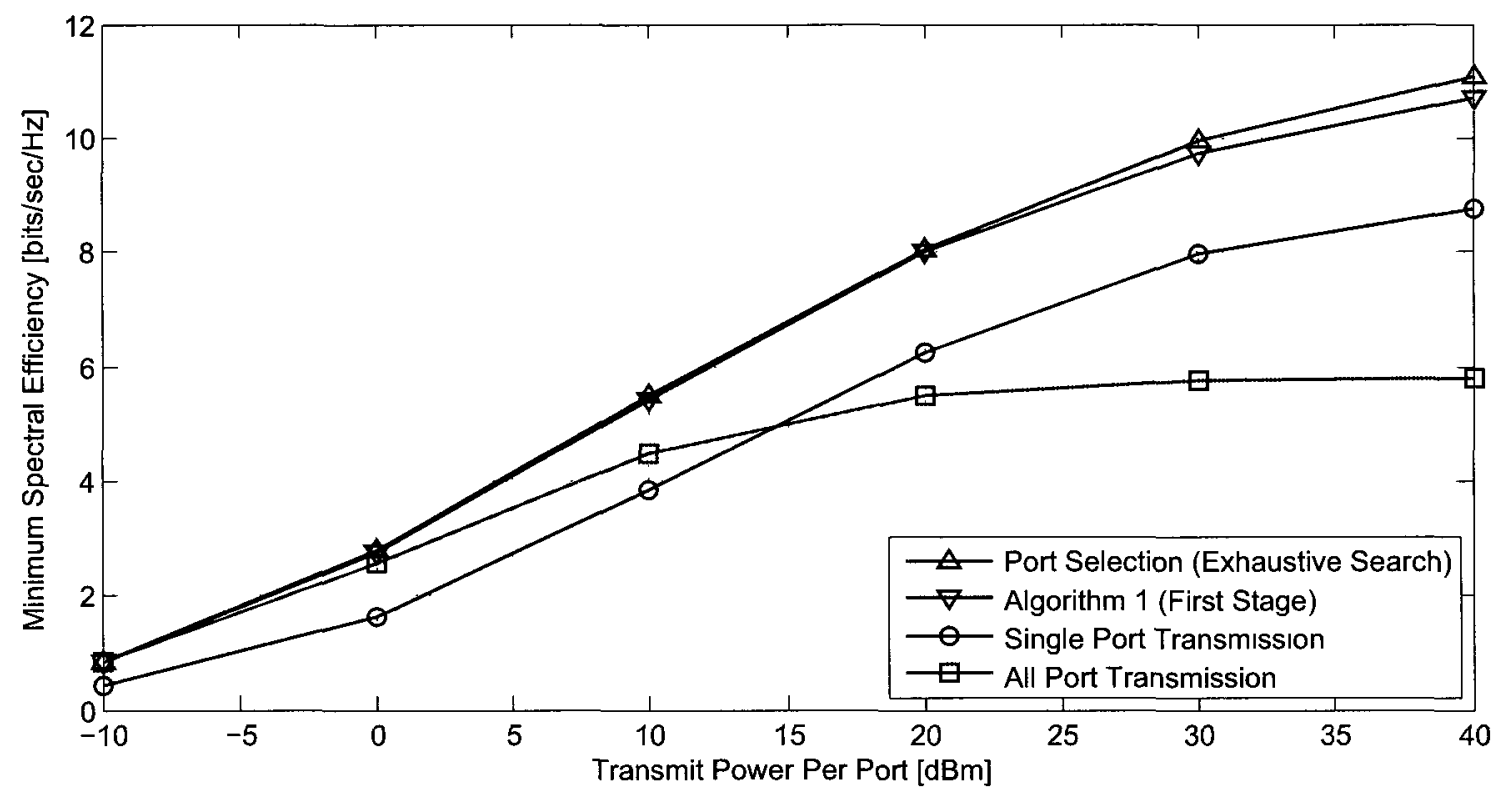

Figure 4.3: A comparison between the largest minimum spectral efficiency achieved by port selection with exhaustive search and with the technique proposed in the first stage, and that achieved by the one-port (without coordination) and all-port strategies for a two-cell cluster in the SMa scenario.

baseline transmission strategies, and also the largest minimum SINR achieved by the CAS architecture. The reported results are averaged over 5000 independent channel realizations. For such a system, obtaining the optimal solution using exhaustive search is computationally prohibitive since this search involves $2^{49} \approx 5.63 \times 10^{14}$ port state vectors. However, the proposed port selection technique can provide close-tooptimal solutions in polynomial time as discussed in Section 4.8. For this technique, the number of Gaussian samples, $J_{1}$, is chosen to be 300 . Furthermore, to enable a fair comparison between the DAS and CAS architectures, the CAS considered herein consists of $L$ antennas at each BS, each of which transmits at the same power, $P$, as each active port in the DAS. Additionally, all $L$ antennas are used to serve the intended UT, and co-phasing is performed at each antenna.

It can be seen from Fig. 4.4 that the proposed technique outperforms the two baseline transmission strategies and the CAS in terms of the largest minimum ergodic 
and outage SINR. This gain in performance is particularly significant at higher values of $P$, as seen from both the ergodic and outage results.

In Fig. 4.5, a comparison similar to the one in Fig. 4.4 is considered, except that the performance metric in this figure is the largest minimum spectral efficiency, i.e., the spectral efficiency of the UT with the minimum SINR. A behaviour similar to the one in Fig. 4.4 is observed in Fig. 4.5, with the proposed technique outperforming the baseline transmission strategies and the CAS, especially at higher values of $P$. For instance, when $P=20 \mathrm{dBm}$, the largest minimum spectral efficiency achieved by the proposed technique at an outage probability of 0.1 is approximately $350 \%, 800 \%$, and $2800 \%$ greater than those achieved by the single-port transmission strategy, the all-port transmission strategy, and the CAS architecture, respectively.

In Fig. 4.6, the distribution of the average number of ports that are activated per cell by the proposed port selection technique is shown for different values of $P$. In Fig. 4.7, the mean values corresponding to each $P$ is shown. It can be observed from these figures that the number of active ports is relatively high at low values of $P$, and decreases as $P$ increases. This observation is consistent with the performance results depicted in Figs. 4.4(a) and 4.5(a), wherein the all-port transmission strategy outperforms the single-port one when $P$ is low, and vice versa when $P$ is high. The results in Fig. 4.7 also indicate that the proposed port selection technique leads to significant power savings by deactivating a considerable percentage of the available ports when $P$ is relatively high.

In Fig. 4.8, the ergodic and outage average spectral efficiency per cell achieved by the proposed port selection technique is compared with that achieved by the two baseline transmission strategies and the CAS. It can be seen from the figure that the performance of the proposed technique is comparable to that of the baseline strategies, despite the fact that this technique is not necessarily optimal for this metric. Furthermore, the performance gain over the CAS is maintained. 


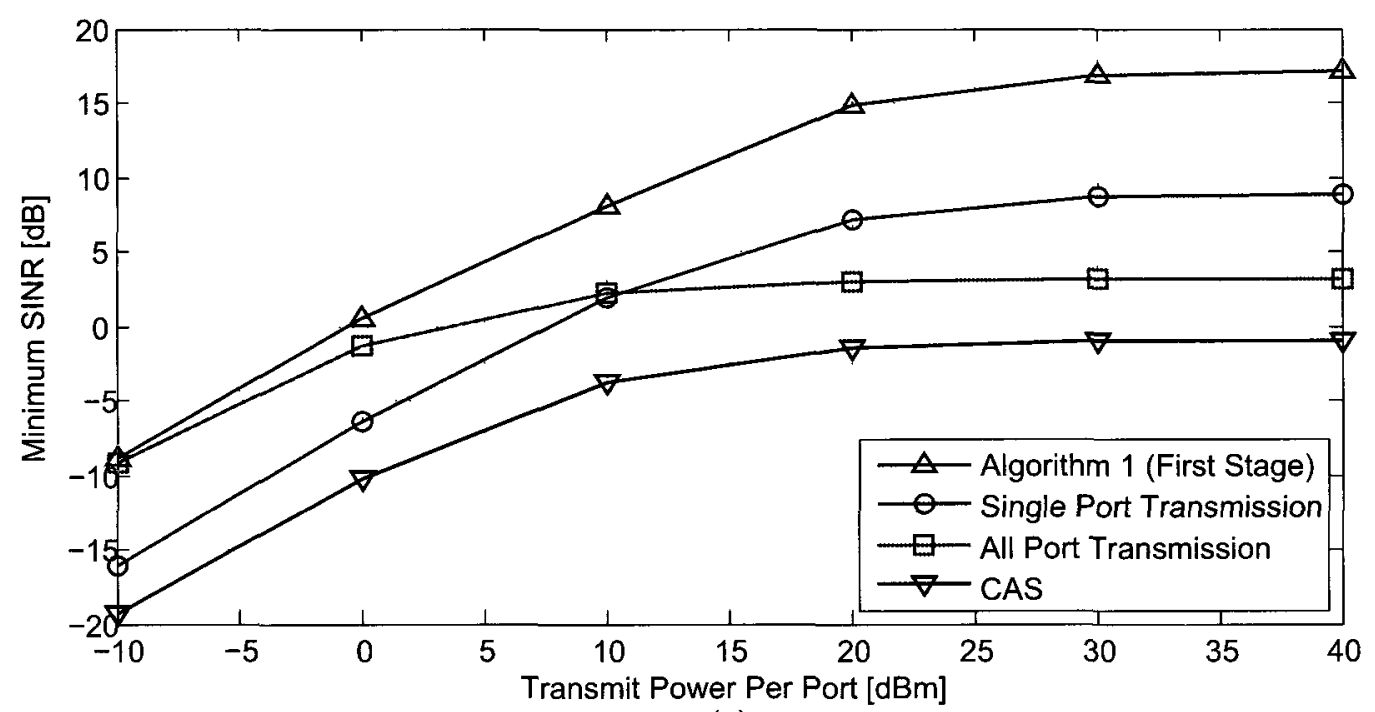

(a)

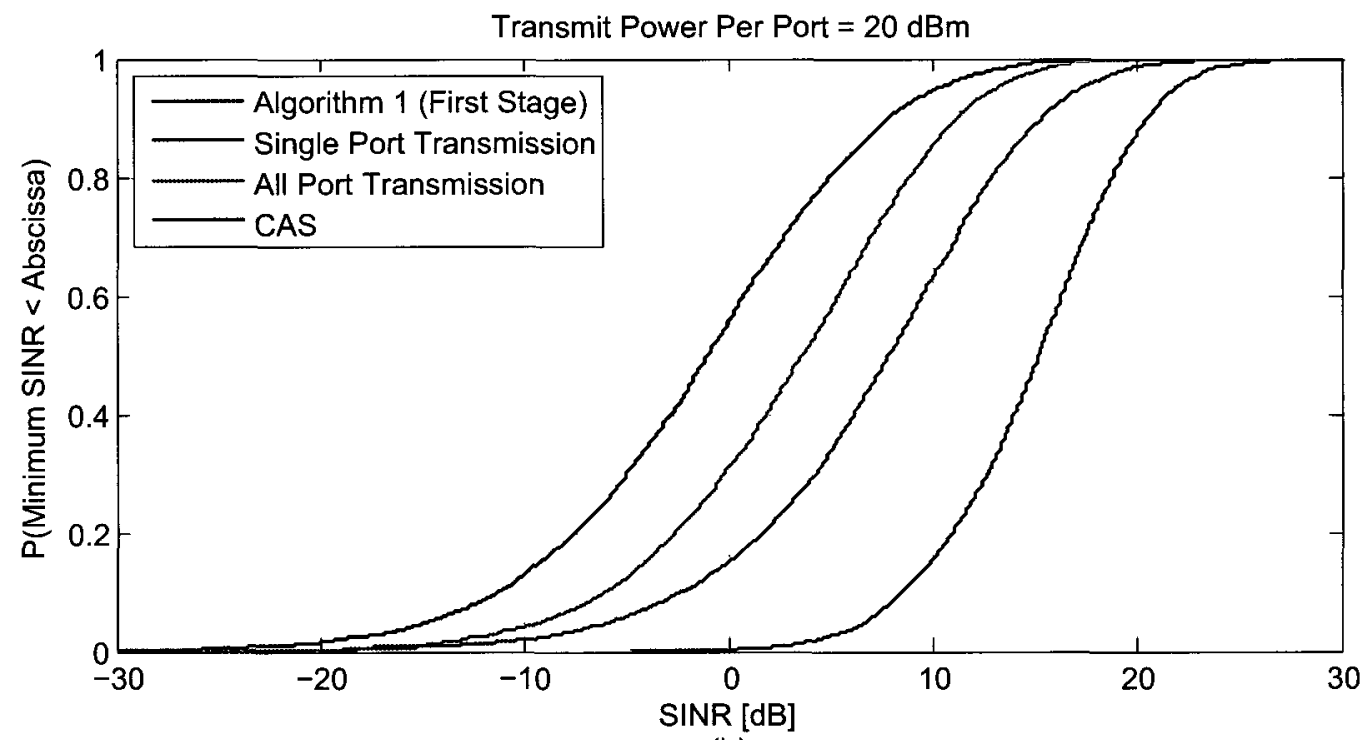

(b)

Figure 4.4: A comparison between the largest minimum SINR achieved by the port selection technique proposed in the first stage, and that achieved by the one-port (without coordination) and all-port strategies for a seven-cell cluster in the SMa scenario. 


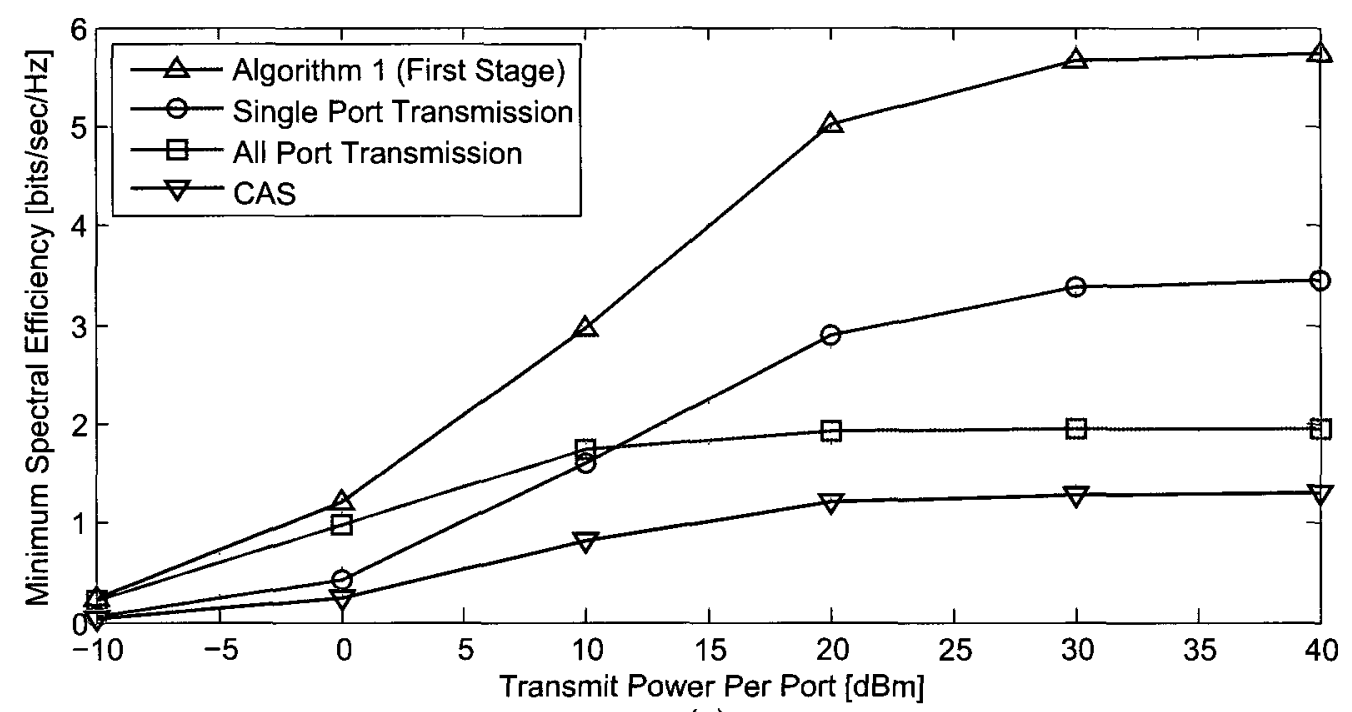

(a)

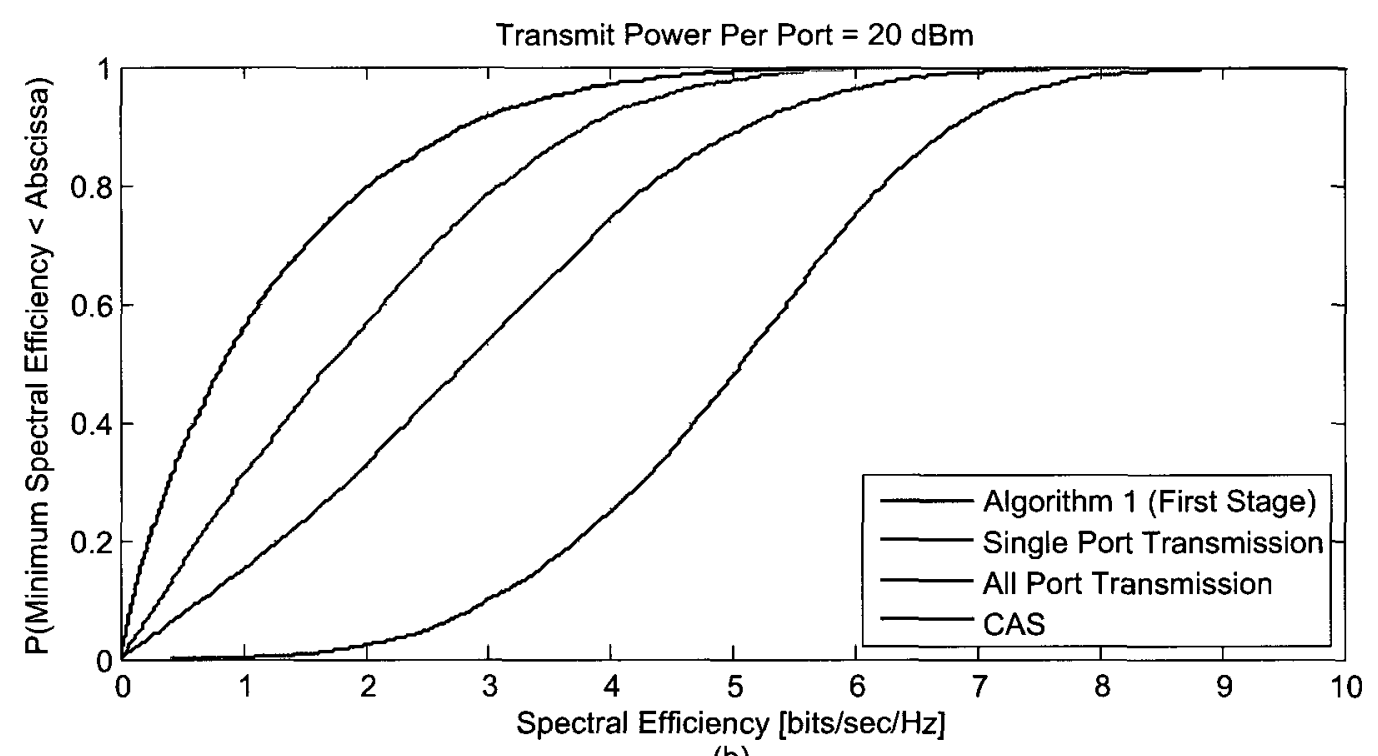

(b)

Figure 4.5: A comparison between the largest minimum spectral efficiency achieved by the port selection technique proposed in the first stage, and that achieved by the one-port (without coordination) and all-port strategies for a seven-cell cluster in the SMa scenario. 


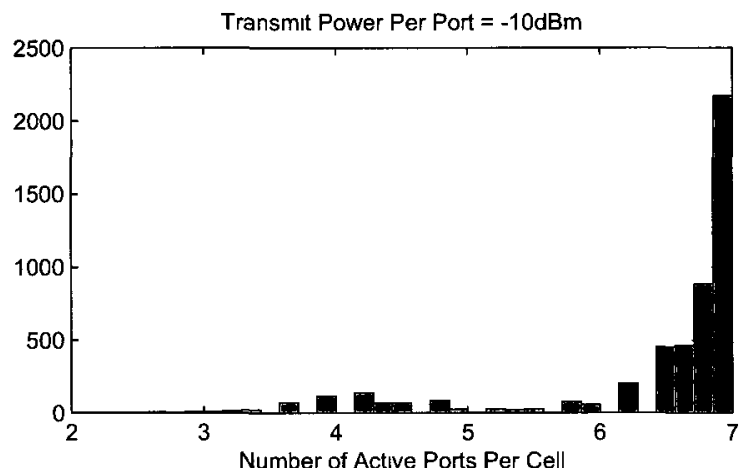

(a)

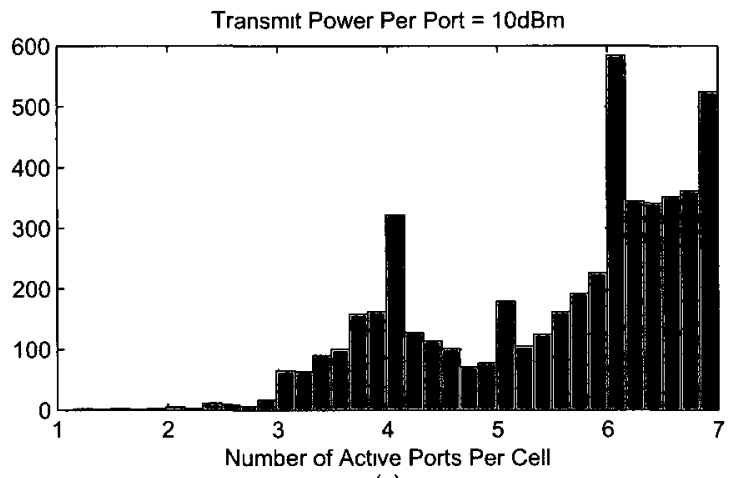

(c)

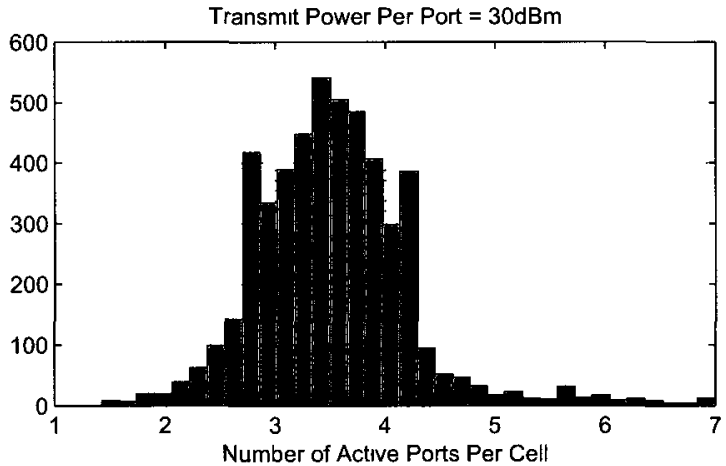

(e)

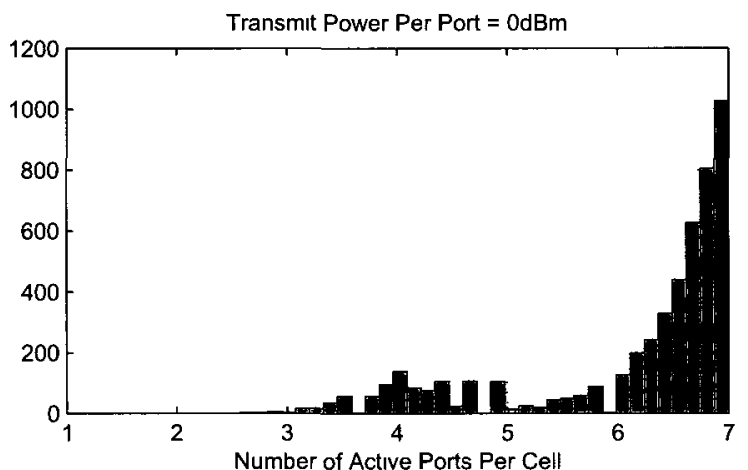

(b)

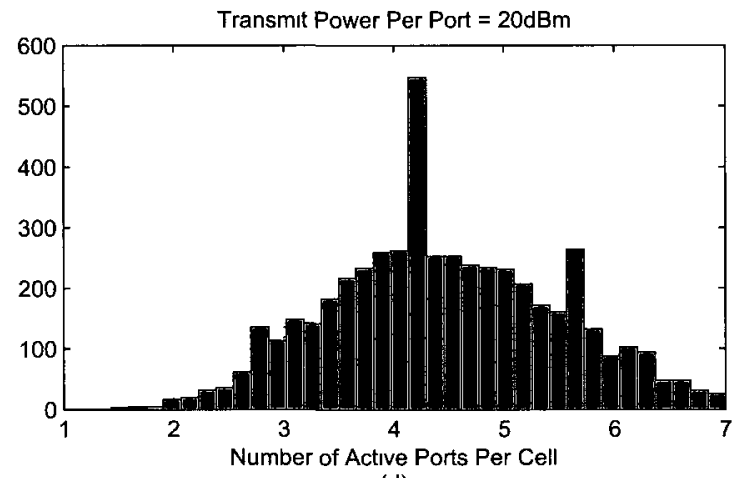

(d)

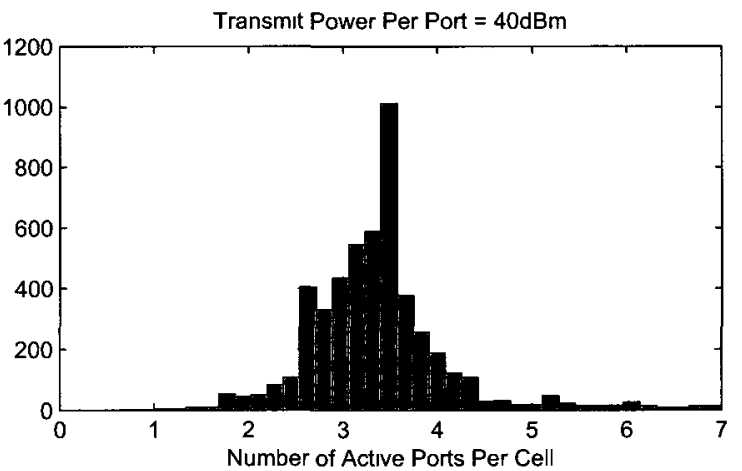

(f)

Figure 4.6: The distribution of the number of ports activated per cell by the port selection technique proposed in the first stage for a seven-cell cluster in the SMa scenario. 


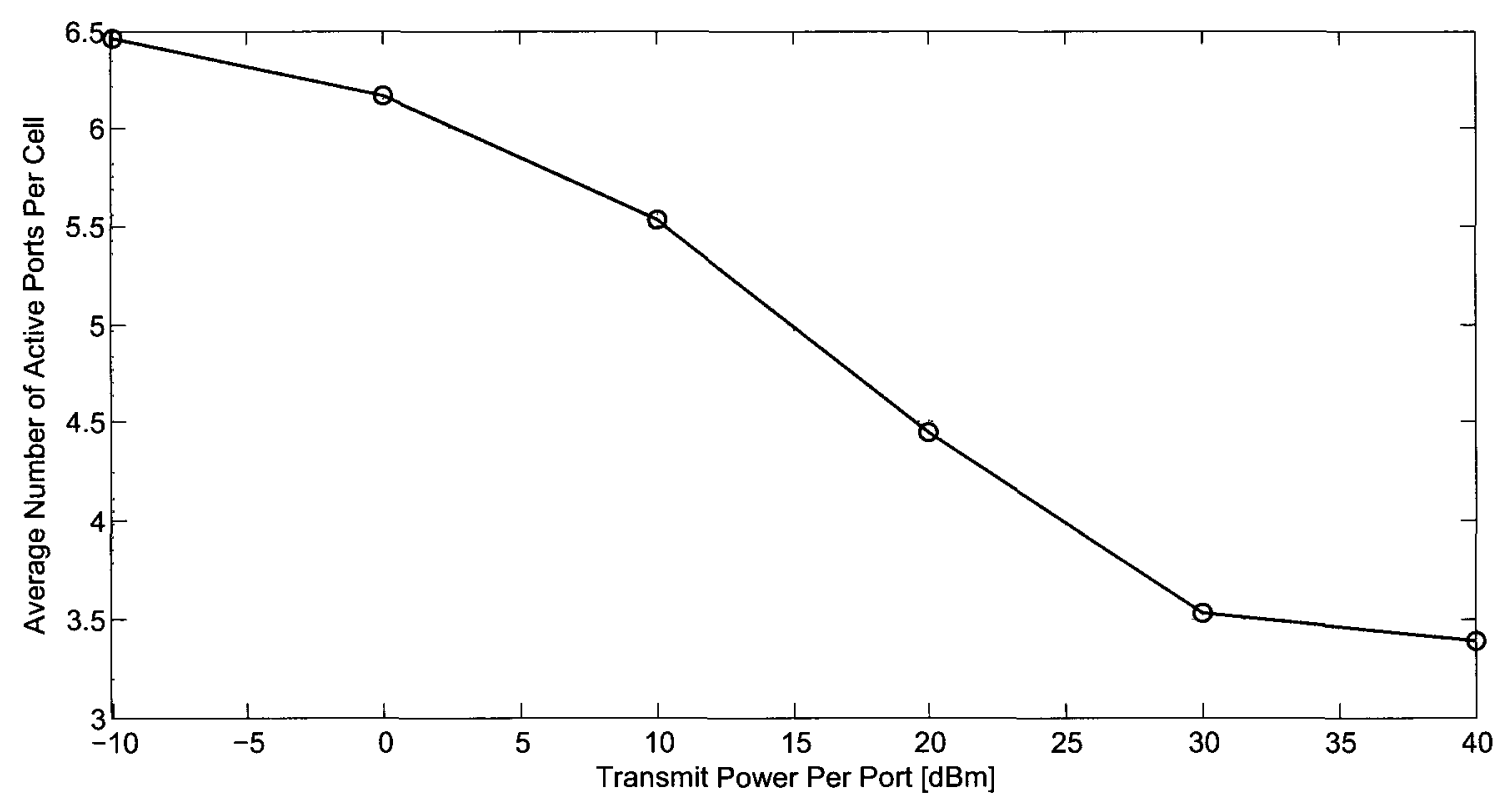

Figure 4.7: The average number of ports activated per cell by the port selection technique proposed in the first stage for a seven-cell cluster in the SMa scenario.

An important observation from the results presented in this example is that, despite the fact that the proposed port selection technique generally uses fewer than $L$ antenna ports in each cell, its performance is significantly better than that of the CAS, which uses all $L$ antennas for transmission. Hence, the DAS employing the proposed technique leads to simultaneous power savings and performance gains over the corresponding CAS.

Example 4.3. In this example, a comparison similar to the one in Example 4.2 is considered, but for the UMa scenario. The results corresponding to those in Figs. 4.4, 4.5, 4.6, 4.7, and 4.8 are shown in Figs. 4.9, 4.10, 4.11, 4.12, and 4.13, respectively.

Comparing the results in Fig. 4.9 with the corresponding results in Fig. 4.4, it can be seen that, at lower values of $P$, the achieved minimum SINRs are higher for the UMa scenario than the SMa one, and vice versa at higher values of $P$. A similar observation is made in comparing the achieved minimum spectral efficiencies 


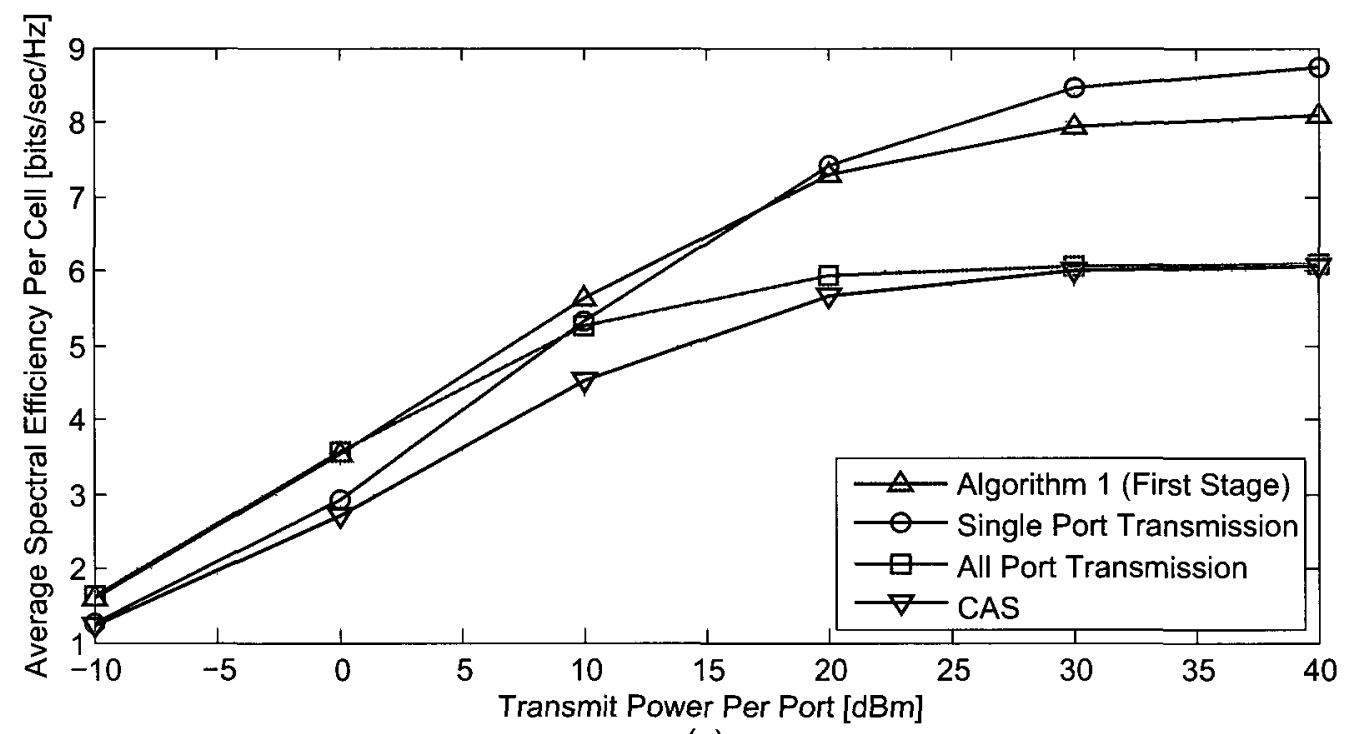

(a)

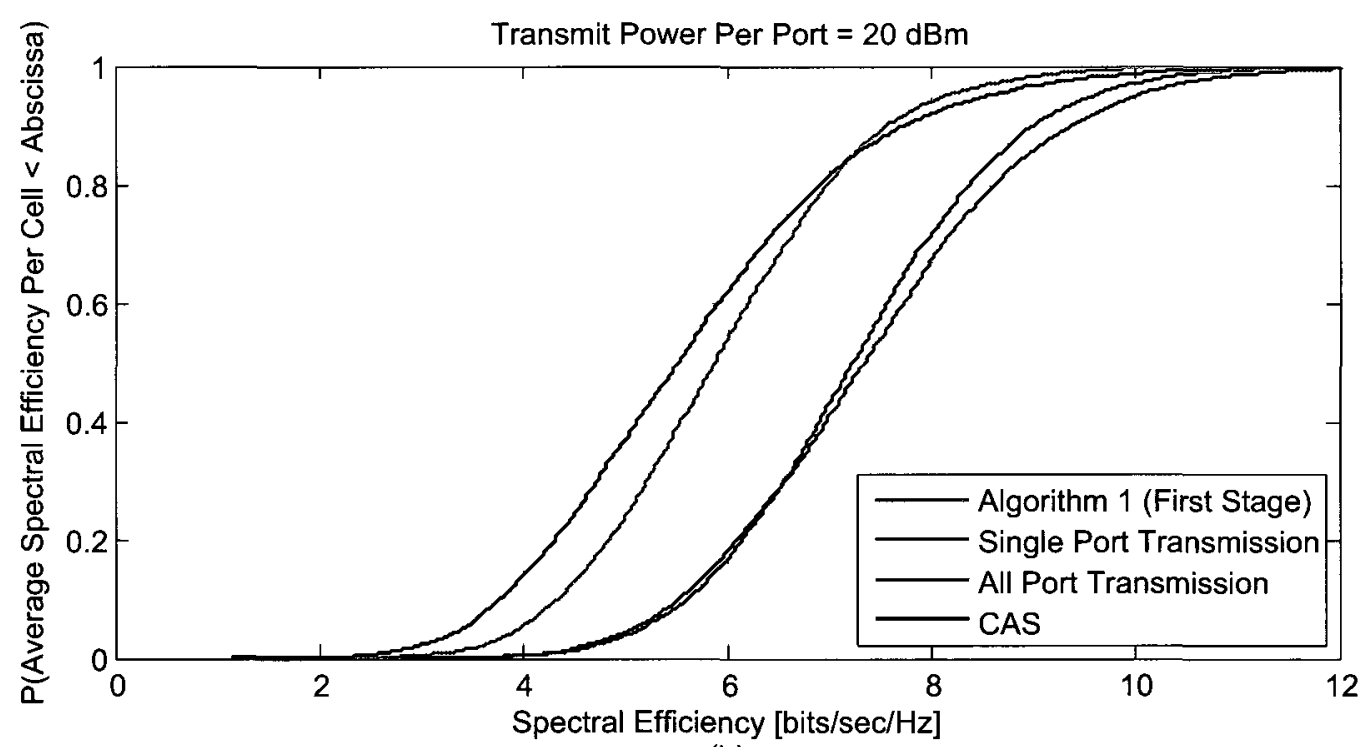

(b)

Figure 4.8: A comparison between the average spectral efficiency per cell achieved by the port selection technique proposed in the first stage, by the single-port (without coordination) and all-port transmission strategies, and by the CAS for a seven-cell cluster in the SMa scenario. 
in Figs. 4.10 (UMa scenario) and 4.5 (SMa scenario). This behaviour can be attributed to the fact that at low values of $P$, the system is noise-limited, whereas at high values of $P$, it is interference-limited.

From both Figs. 4.9(a) and 4.10(a), it can also be noticed that when $P$ is low, the performance of the proposed technique is close to that of the all-port transmission strategy. This observation suggests that when the power per port is low, the SINRmaximizing strategy is to use a greater number of ports for transmission in each cell. This effect is further demonstrated in Figs. 4.11 and 4.12, where it can be observed that the number of active ports per cell approaches $L$ as $P$ decreases.

In Fig. 4.13, the ergodic and outage average spectral efficiency per cell achieved by the proposed port selection technique is compared with that achieved by the two baseline transmission strategies and the CAS. It can be seen in this figure that, similar to Fig. 4.8, the proposed port selection technique achieves comparable performance to that of the all-port transmission strategy when $P$ is low, and that of the one-port transmission strategy when $P$ is high. Furthermore, despite being sub-optimal for this metric, the proposed technique outperforms the CAS even though it uses fewer ports, and hence, less power.

Example 4.4. In this example, the performance of the two-stage approach proposed in Section 4.7 is evaluated for a system with $M=2$ in the SMa scenario, and compared with a computationally-expensive close-to-optimal joint solution. Due to the complexity of the latter approach, a single channel realization is simulated. The channel gains for this realization are provided in Table 4.6.

The close-to-optimal solution is obtained through exhaustive search over all possible port state vectors, and by using the technique proposed in Section 4.6 to generate close-to-optimal beam steering coefficients for each vector. For the proposed twostage approach, 100 Gaussian samples are generated in the first and second stages; i.e., $J_{1}=J_{2}=100$. 


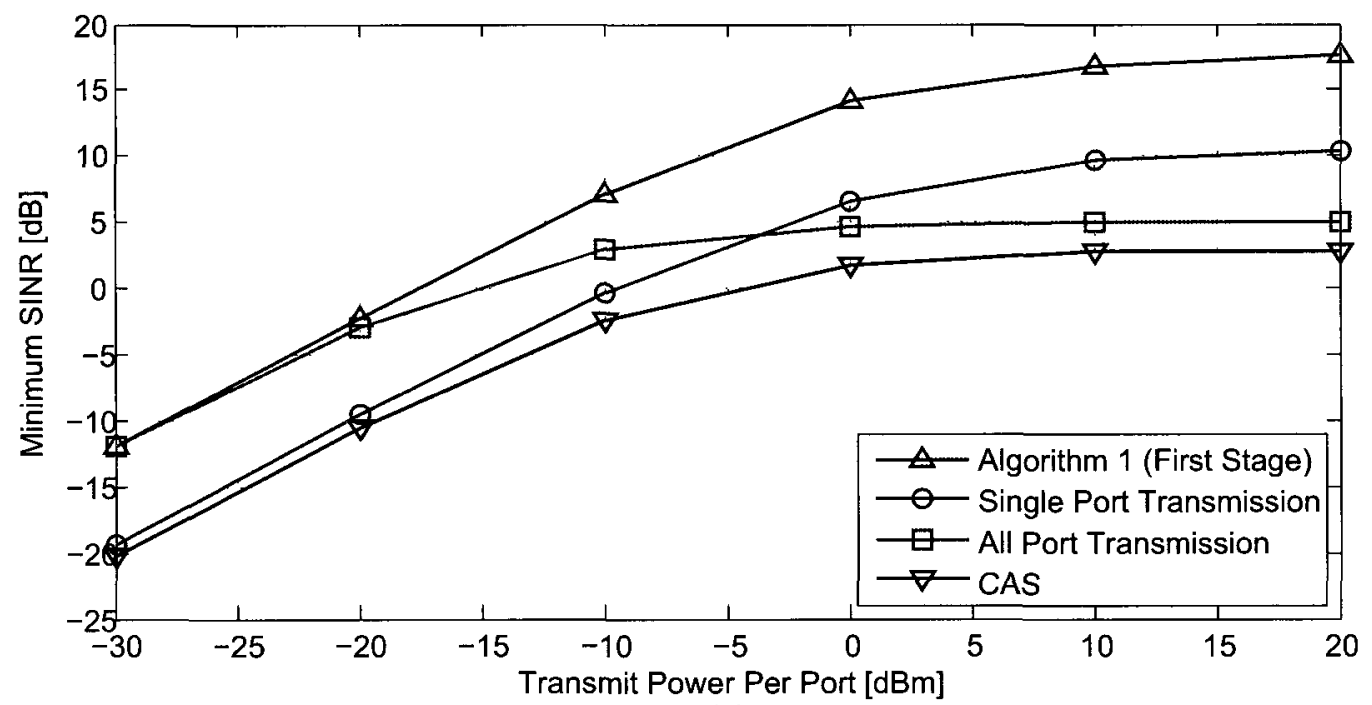

(a)

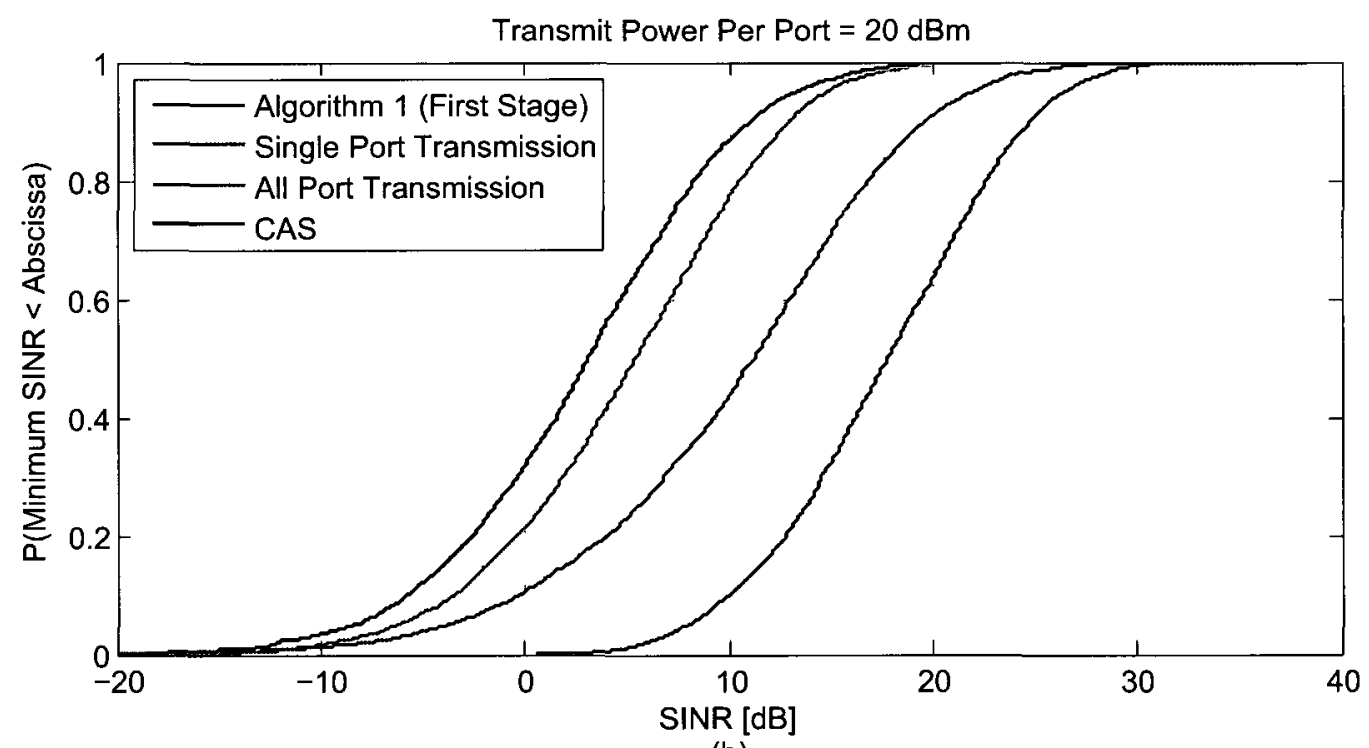

(b)

Figure 4.9: A comparison between the largest minimum SINR achieved by the port selection technique proposed in the first stage, and that achieved by the one-port (without coordination) and all-port strategies for a seven-cell cluster in the UMa scenario. 


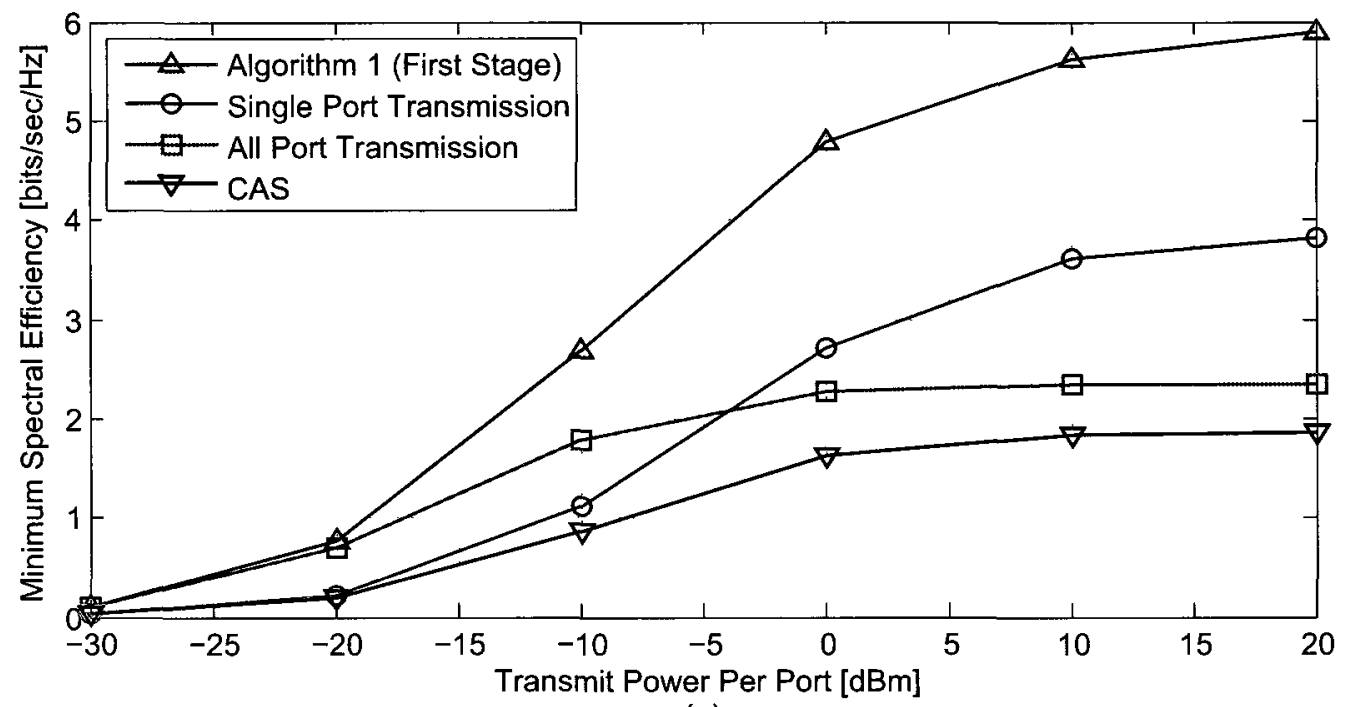

(a)

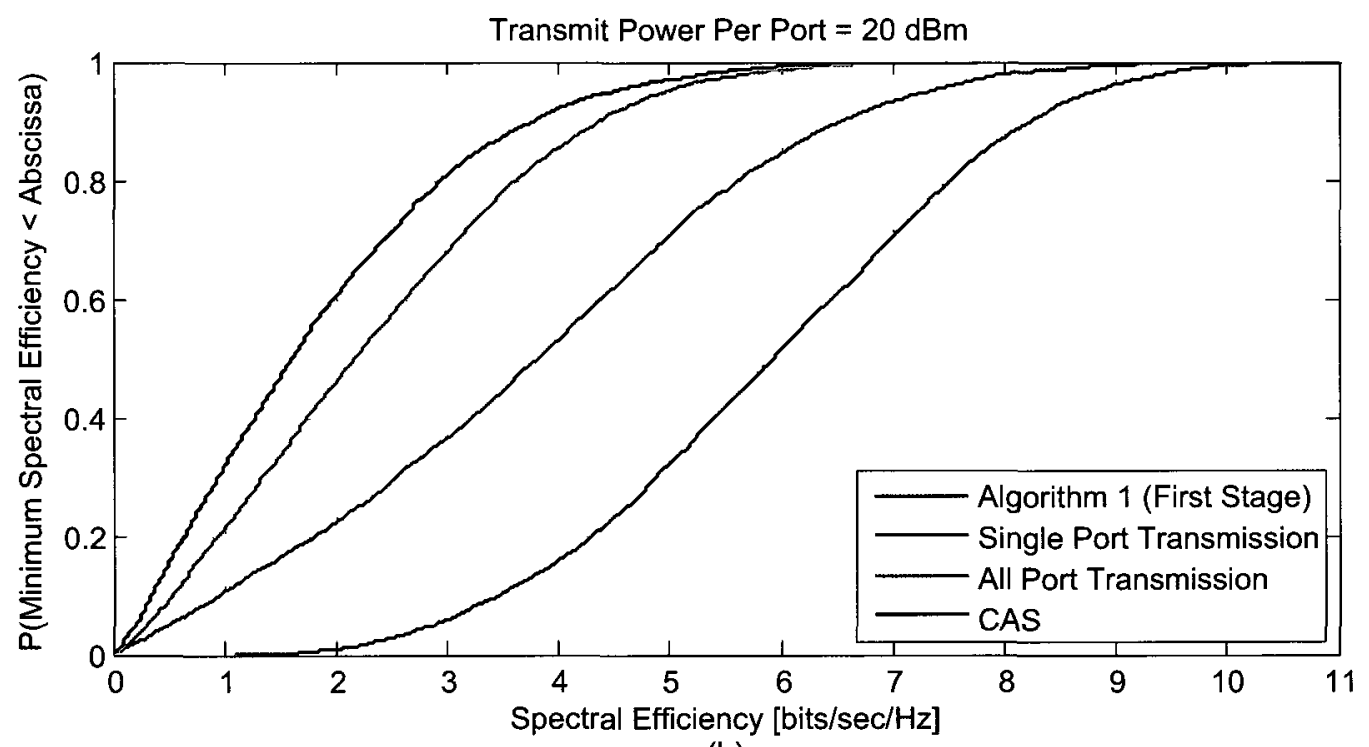

(b)

Figure 4.10: A comparison between the largest minimum spectral efficiency achieved by the port selection technique proposed in the first stage, and that achieved by the one-port (without coordination) and all-port strategies for a seven-cell cluster in the UMa scenario. 


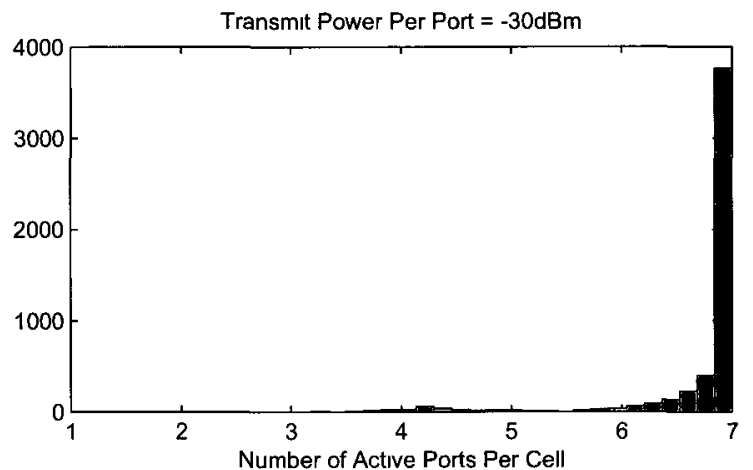

(a)

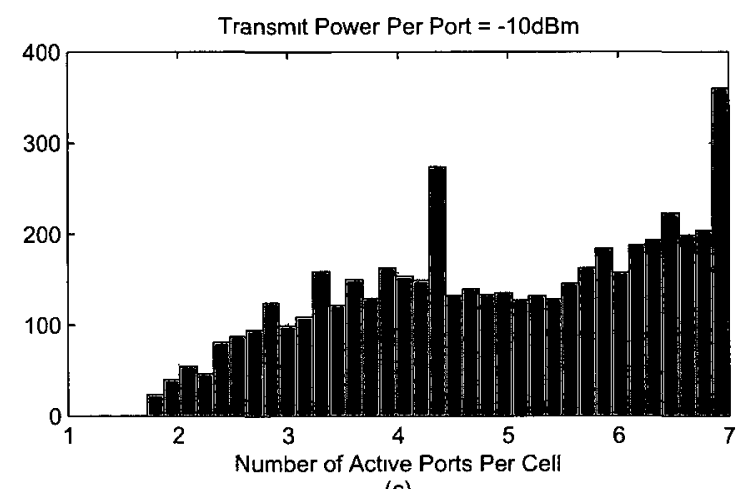

(c)

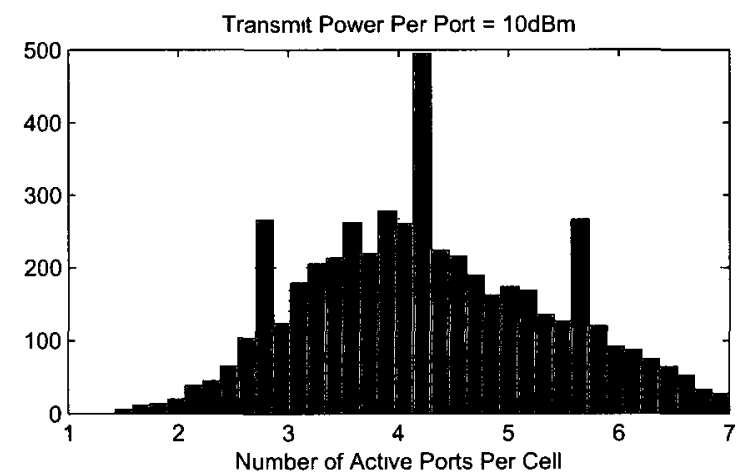

(e)

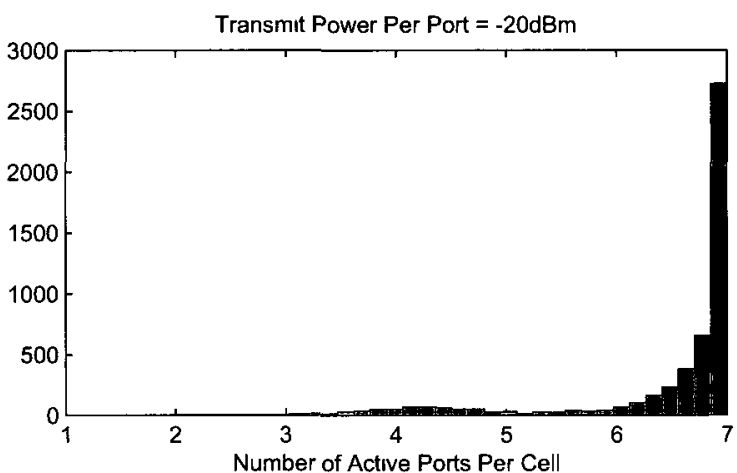

(b)

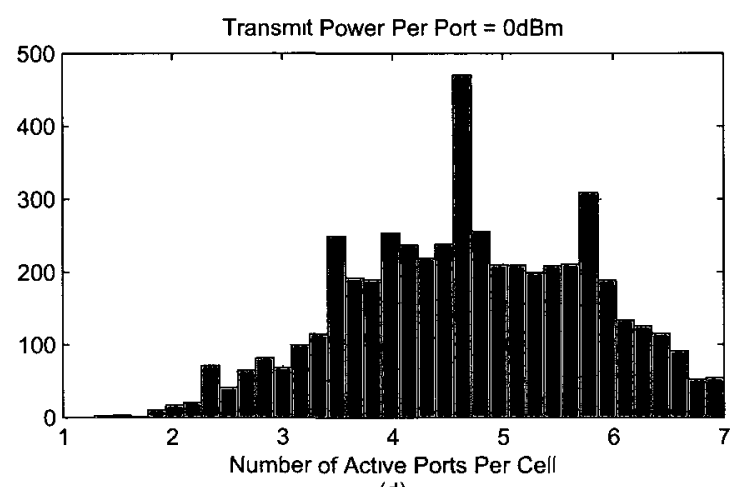

(d)

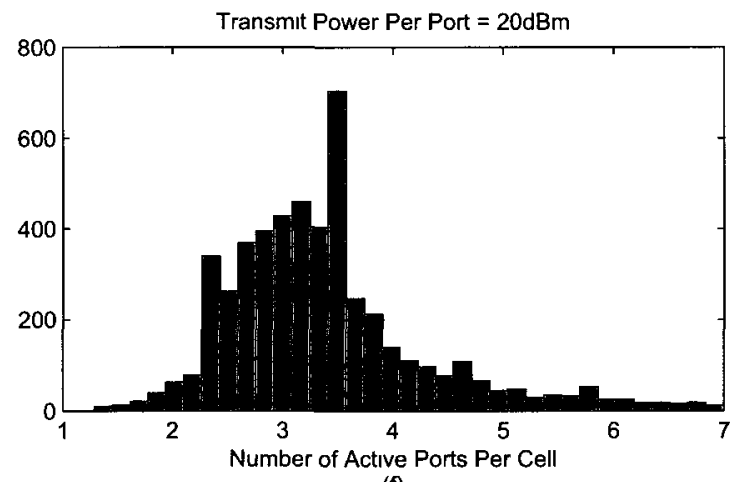

(f)

Figure 4.11: The distribution of the number of ports activated per cell by the port selection technique proposed in the first stage for a seven-cell cluster in the UMa scenario. 


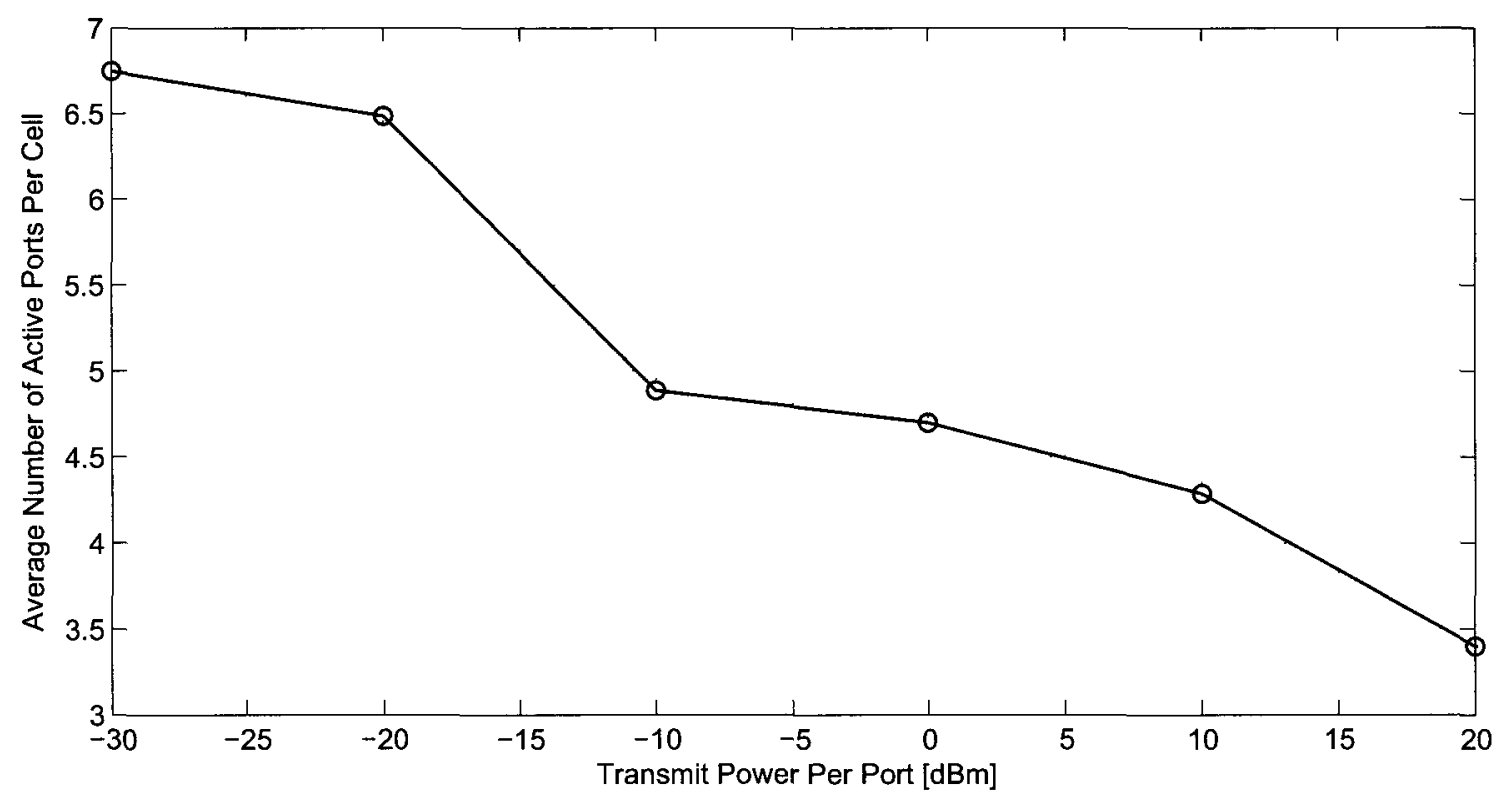

Figure 4.12: The average number of ports activated per cell by the selection technique proposed in the first stage for a seven-cell cluster in the UMa scenario.

Figures 4.14 and 4.15 show the largest minimum SINRs and corresponding spectral efficiencies, respectively, that are achieved by the two-stage approach in Section 4.7 with $\hat{J}=10,50$, and 100, and by the exhaustive search described above. For comparison, in these figures, the largest minimum SINRs and corresponding spectral efficiencies achieved by the techniques in Sections 4.5 and 4.6 are also shown. From the figures, it can be seen that the performance of the proposed two-stage technique approaches that of the close-to-optimal joint solution as $\hat{J}$ increases. Both techniques perform significantly better than the techniques presented in Sections 4.5 and 4.6 for the given initial beam steering coefficients and the port state vector generated in the first stage, respectively. 


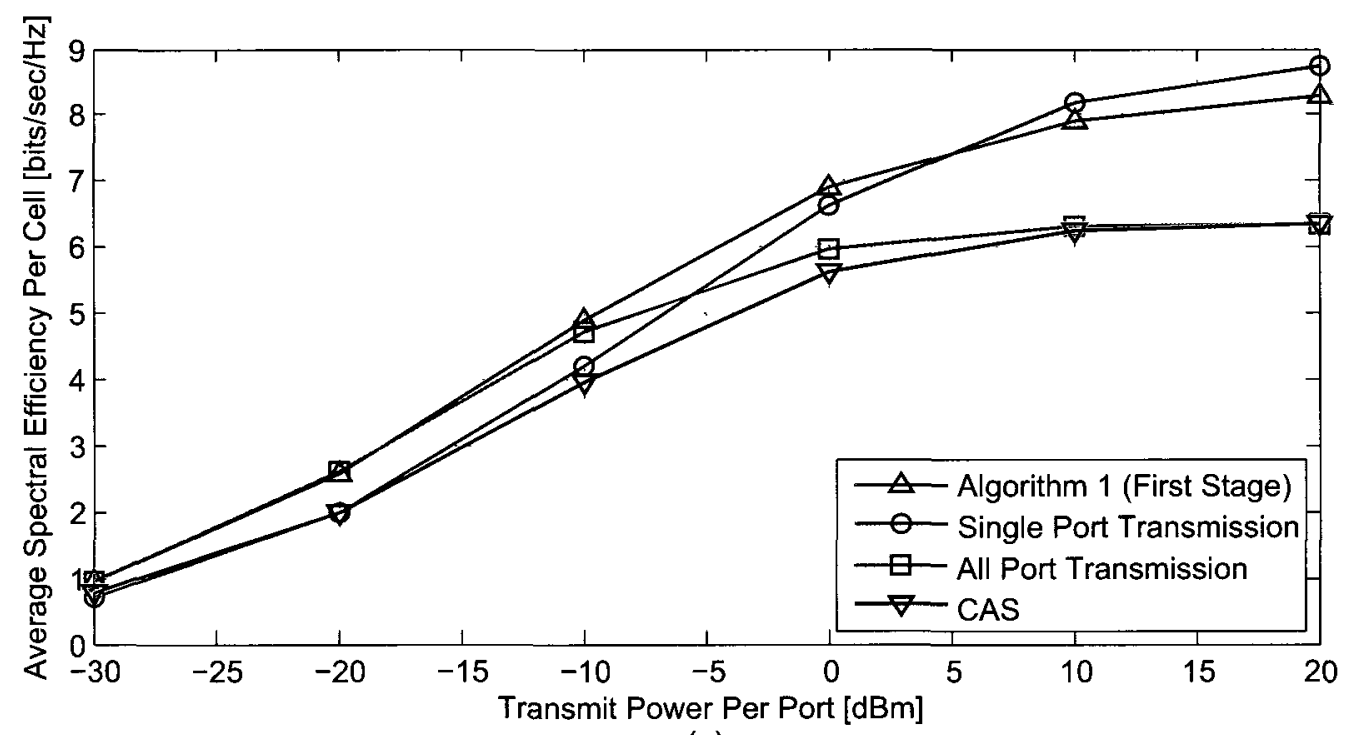

(a)

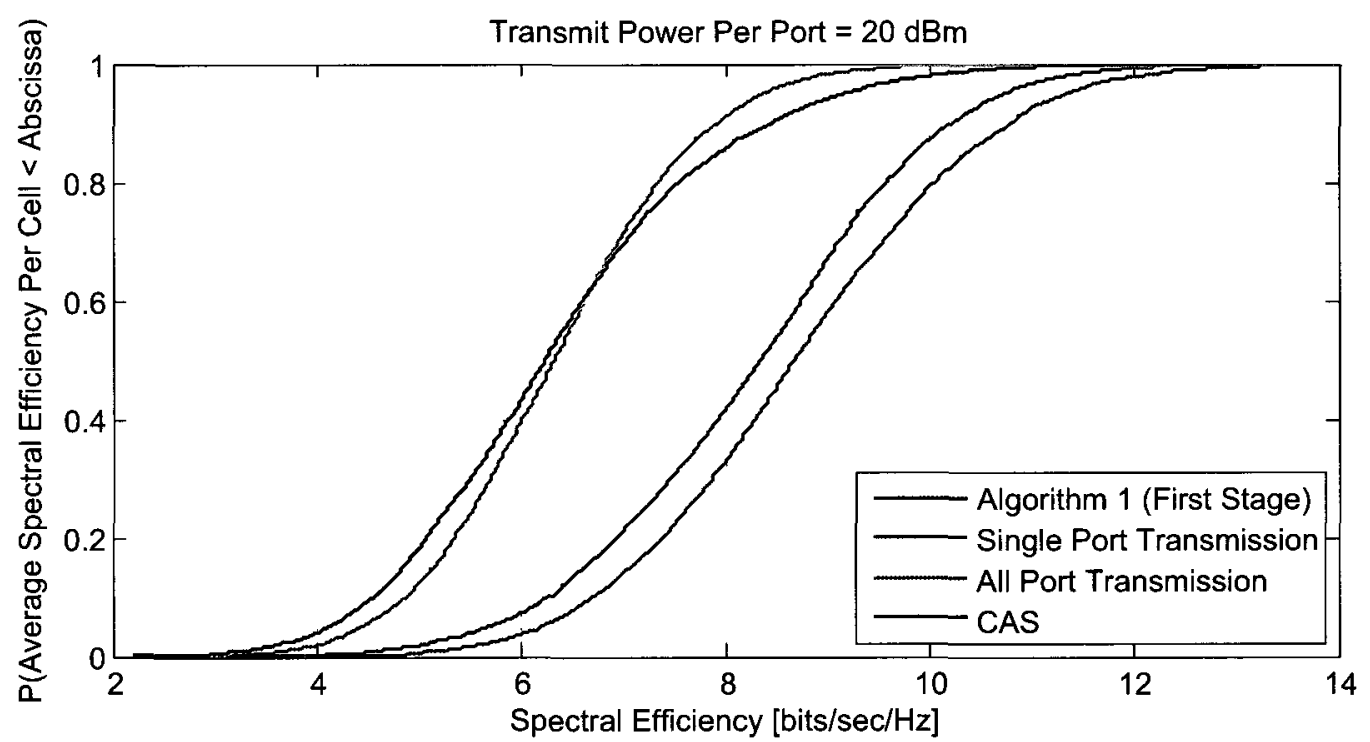

(b)

Figure 4.13: A comparison between the average spectral efficiency per cell achieved by the port selection technique proposed in the first stage, by the single-port (without coordination) and all-port transmission strategies, and by the CAS for a seven-cell cluster in the UMa scenario. 


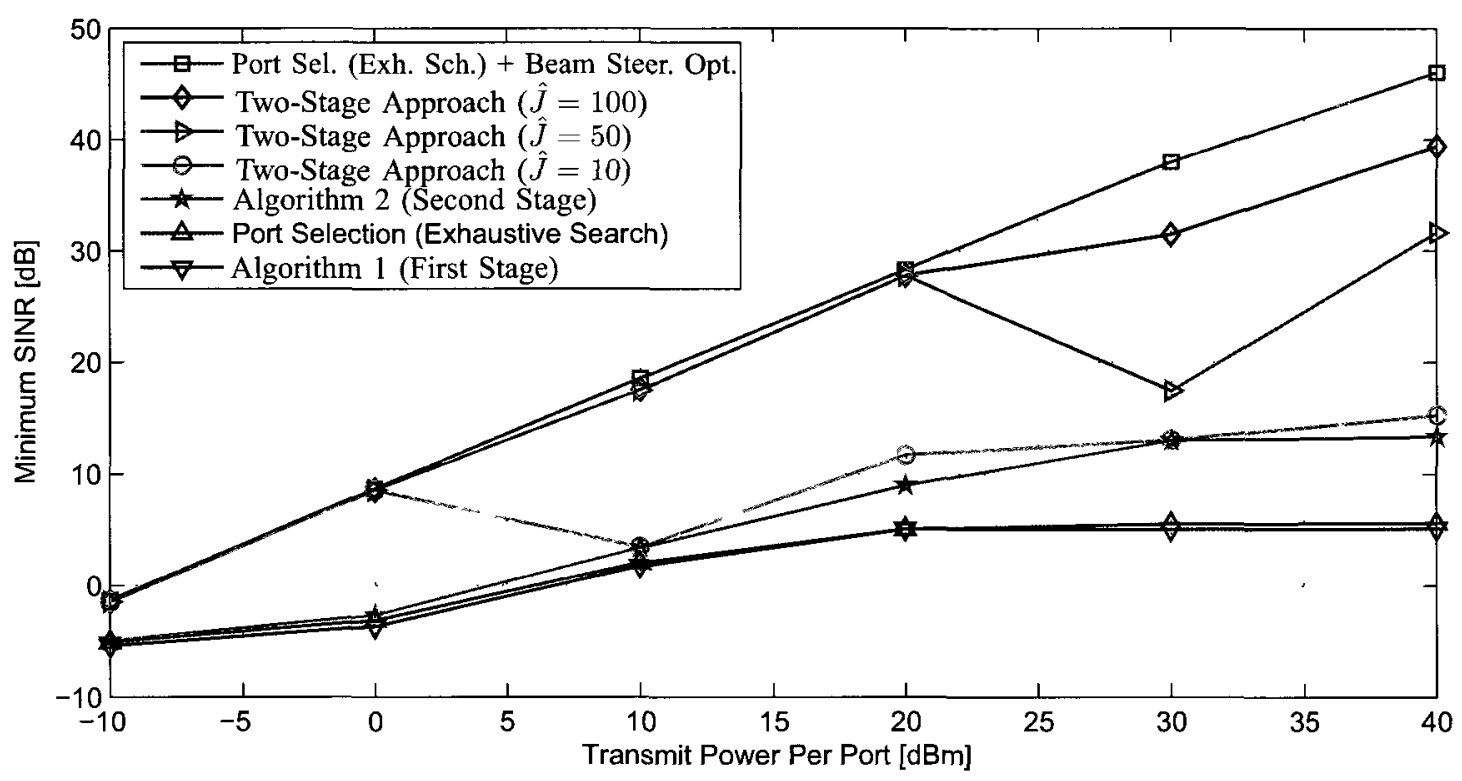

Figure 4.14: A comparison between the largest minimum SINR achieved by the computationally-efficient two-stage approach proposed herein, an exhaustive-search based close-to-optimal solution, and the port selection technique in the first stage for a two-cell cluster in the SMa scenario.

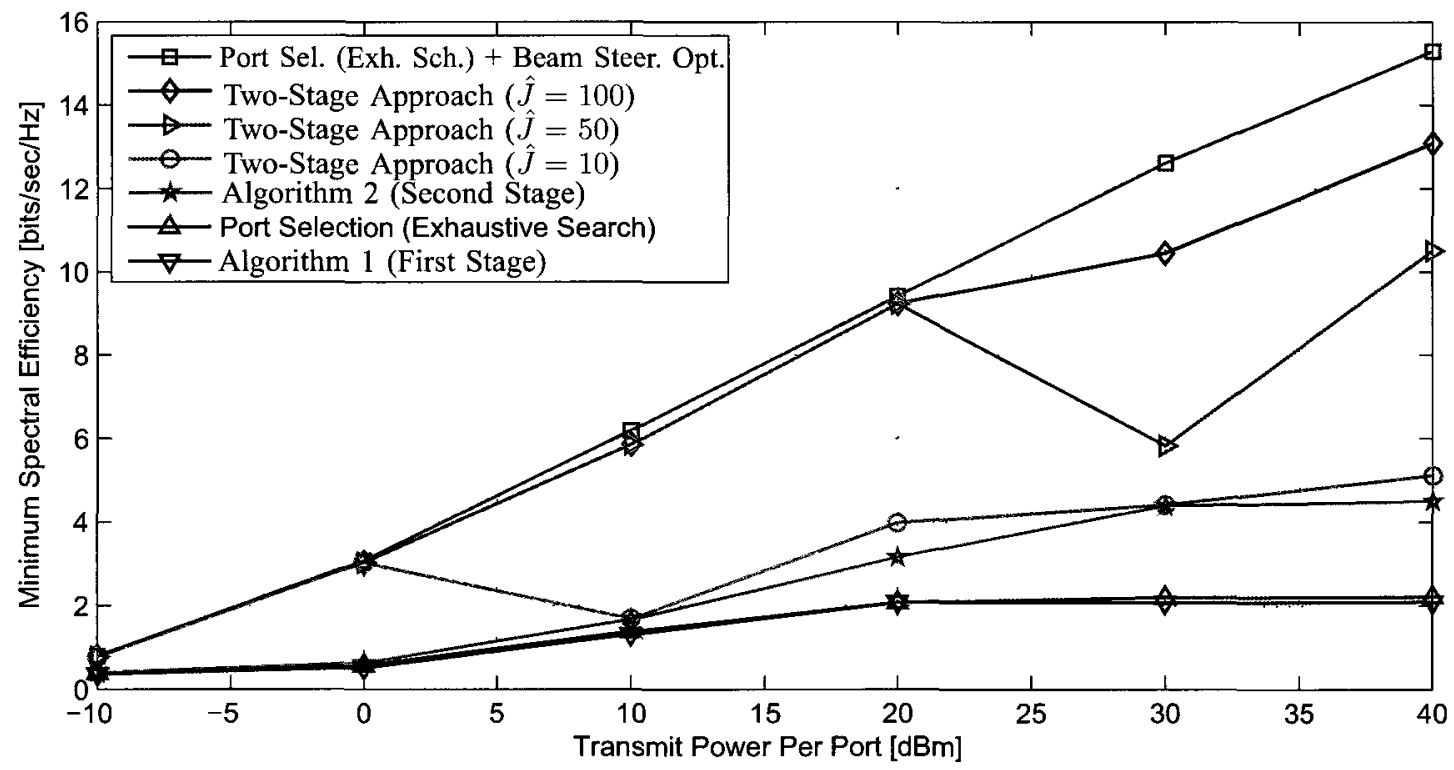

Figure 4.15: A comparison between the largest minimum spectral efficiency achieved by the computationally-efficient two-stage approach proposed herein, an exhaustivesearch based close-to-optimal solution, and the port selection technique in the first stage for a two-cell cluster in the SMa scenario. 
Table 4.6: Channel gains between the ports and the UTs in each cell $\left(\times 10^{-4}\right)$

\begin{tabular}{|c|rr|rr|}
\hline \multirow{2}{*}{ Port \# } & \multicolumn{2}{|c|}{ UT in the 1st cell } & \multicolumn{2}{c|}{ UT in the 2nd cell } \\
& 1st Cell & 2nd Cell & 1st Cell & 2nd Cell \\
\hline 1 & $-0.0083-j 0.0042$ & $-0.0025-j 0.0132$ & $-0.0005-j 0.0003$ & $-0.0053-j 0.0284$ \\
2 & $-0.2329+j 0.1166$ & $0.0261-j 0.0021$ & $-0.0043+j 0.0021$ & $0.0580-j 0.0047$ \\
3 & $0.0187-j 0.0141$ & $0.0002-j 0.0015$ & $0.0063-j 0.0047$ & $0.0008-j 0.0052$ \\
4 & $-0.0013+j 0.0083$ & $-0.0009+j 0.0022$ & $-0.0022+j 0.0140$ & $-0.0058+j 0.0141$ \\
5 & $0.0030+j 0.0010$ & $0.0005-j 0.0000$ & $0.0020+j 0.0007$ & $0.0128-j 0.0006$ \\
6 & $0.0024+j 0.0154$ & $-0.0001-j 0.0010$ & $0.0006+j 0.0035$ & $-0.0033-j 0.0415$ \\
7 & $0.1053+j 0.1351$ & $-0.0017+j 0.0039$ & $0.0098+j 0.0126$ & $-0.0152+j 0.0342$ \\
\hline
\end{tabular}




\section{Chapter 5}

\section{Conclusions and Future Work}

\section{$5.1 \quad$ Summary}

In this thesis, CoMP transmission schemes are integrated into the DAS architecture. In the first part of the thesis, two coordinated transmission schemes, namely DAS ZF-DPC and DAS BD, are developed to serve multiple UTs in a particular RB in each cell with no intra-cell interference. These schemes are also extended to the multi-cell processing case, wherein a central processing entity is used to coordinate the transmission between ports in a cluster of cells, which is subject to a total power constraint. Simulation is used to demonstrate the performance gains of the DAS as compared to the CAS, and also those attained by using centralized multi-cell processing instead of single-cell processing.

In the second part of the thesis, a coordinated multi-cell DAS is considered, wherein the transmit power of each port is fixed. For this system, the problem of jointly selecting ports and optimizing their beam steering coefficients to maximize the minimum SINR of the UTs is considered. This problem is NP-hard. A two-stage approach is proposed to circumvent this difficulty. In each stage, a sub-problem of the original joint optimization problem is considered. This problem is also NP-hard, and the SDR-based Gaussian randomization technique is used to generate close-tooptimal solutions in polynomial time. Furthermore, the two-stage approach provides an approximate solution to the joint optimization problem in polynomial time. The 
efficacy of the proposed approach is demonstrated using simulation results. Furthermore, it is shown that proper port selection not only results in improved performance, but also provides significant power saving opportunities in the cellular network.

\subsection{Contributions}

- The ZF-DPC and BD schemes are extended to the DAS architecture for intracell and inter-cell interference mitigation.

- The joint selection of ports and their corresponding beam steering coefficients that maximize the minimum SINR, which is an NP-hard optimization problem, is tackled using the SDR-based Gaussian randomization technique.

- The proposed two-stage approach generates an approximate solution to the joint optimization problem in polynomial time, and also provides significant power savings (by deactivating selected ports) without compromising performance.

\subsection{Future Work}

The following is a list of potential topics for future research that are related to the work presented in this thesis. These topics are organized based on relevance to the two main chapters in the thesis.

- Chapter 3:

- The design of transmission schemes such as DAS ZF-DPC and DAS BD with a per-port or per-antenna power constraint.

- The development of a distributed multi-cell processing algorithm, which, although is likely to be sub-optimal as compared to centralized multi-cell processing, would alleviate the load on the backbone network and loosen the CSI requirements for each BS. 
- An investigation of the effect of the number of ports in each cell and their layout on the performance of the cellular system.

- Chapter 4:

- The development of an algorithm using the SDR-based Gaussian randomization technique for the case in which the system is subject to a per-port power constraint, instead of the transmit power being fixed for each port.

- The development of an algorithm that would allow the BSs to determine the port states and beam steering coefficients in a distributed manner (to alleviate the requirement of a central processing entity and the related signalling overhead).

- The design of a port selection and beam steering optimization technique that maximizes the aggregate spectral efficiency. The formulation of such an optimization problem would be fundamentally different than the one in Chapter 4, and may not be amenable to the SDR-based Gaussian randomization technique employed herein.

- The joint optimization of the port states, beam steering coefficients, and the UT scheduling for a given frequency-time RB. This is a more general cross-layer design problem that subsumes the optimization problem considered in this thesis, and hence, can be expected to yield better performance.

- The development of a port selection and beam steering optimization technique for serving multiple UTs in each cell in the same RB.

A more general topic of practical importance is the investigation of the performance of CoMP schemes and algorithms such as the ones designed in this thesis in the presence of imperfect CSI. Such imperfections generally arise due to delay resulting from measurement and feedback to the processing entity and/or errors introduced by the feedback medium. 
Appendices 


\section{Appendix A}

\section{Proofs and Derivations for Chapter 4}

\section{A.1 Derivation of the matrix notation in (4.7)}

Consider the desired and interference signals received by the UT in the $m$-th cell. These signals can be collectively written as

$$
\sum_{n=1}^{M}\left(\sum_{\ell=1}^{L} \alpha_{\ell n} \sqrt{P_{\ell n}} h_{\ell n m} w_{\ell n}\right)^{2}=\sum_{n=1}^{M} \sum_{\jmath=1}^{L} \sum_{\ell=1}^{L} \alpha_{\ell n} \sqrt{P_{\ell n}} h_{\ell n m} w_{\ell n} w_{\jmath n}^{*} h_{\jmath n m}^{*} \sqrt{P_{\jmath n}} \alpha_{\jmath n}
$$

The right hand side of the above equation can also be expressed as $\sum_{n=1}^{M} \boldsymbol{\alpha}_{n}^{T} \boldsymbol{A}_{n, m} \boldsymbol{\alpha}_{n}$, where

$$
\boldsymbol{\alpha}_{n}=\left[\begin{array}{c}
\alpha_{1 n} \\
\vdots \\
\alpha_{L n}
\end{array}\right] \text { and } \boldsymbol{A}_{n, m}=\left[\begin{array}{ccc}
\left|h_{1 n m}\right|^{2} P_{1 n} & \ldots & \left|h_{1 n m} h_{L n m}\right| \sqrt{P_{1 n} P_{L n}} \\
\vdots & \ddots & \vdots \\
\left|h_{L n m} h_{1 n m}\right| \sqrt{P_{L n} P_{1 n}} & \cdots & \left|h_{L n m}\right|^{2} P_{L n}
\end{array}\right]
$$

Using this notation, the desired signal term in the numerator and the interference term in the denominator of the SINR expression in (4.4) can be expressed as $\boldsymbol{\alpha}^{T} \boldsymbol{C}_{m} \boldsymbol{\alpha}$ and 
$\boldsymbol{\alpha}^{T} \boldsymbol{D}_{m} \boldsymbol{\alpha}$, respectively, where

$\boldsymbol{\alpha} \triangleq\left[\begin{array}{c}\boldsymbol{\alpha}_{1} \\ \vdots \\ \boldsymbol{\alpha}_{M}\end{array}\right], \boldsymbol{C}_{m} \triangleq \oplus_{\imath=1}^{m-1} \mathbf{0} \oplus \boldsymbol{A}_{m, m} \oplus_{\imath=m+1}^{M} \mathbf{0}$, and $\boldsymbol{D}_{m} \triangleq \oplus_{\imath=1}^{m-1} \boldsymbol{B}_{m, \imath} \oplus \mathbf{0} \oplus_{\imath=m+1}^{M} \boldsymbol{B}_{m, \imath}$

The expression in (4.7) is obtained by using this notation.

\section{A.2 Proof for Lemma 4.1}

Using the definitions of $\boldsymbol{F}_{m}$ and $\boldsymbol{\Psi}$ in (4.11) and (4.14), respectively, we have

$$
\begin{aligned}
\operatorname{Tr}\left(\boldsymbol{F}_{m} \boldsymbol{\Psi}\right) & =\operatorname{Tr}\left(\left[\begin{array}{cc}
\boldsymbol{D}_{m} & \boldsymbol{D}_{m} \mathbf{1} \\
\mathbf{1}^{T} \boldsymbol{D}_{m} & \mathbf{1}^{T} \boldsymbol{D}_{m} \mathbf{1}+4 \sigma^{2}
\end{array}\right]\left[\begin{array}{cc}
\boldsymbol{X} & \boldsymbol{x} \\
\boldsymbol{x}^{T} & 1
\end{array}\right]\right) \\
& =4 \sigma^{2}+\operatorname{Tr}\left(\boldsymbol{D}_{m} \boldsymbol{X}+2 \boldsymbol{x}^{T} \boldsymbol{D}_{m} \mathbf{1}+\mathbf{1}^{T} \boldsymbol{D}_{m} \mathbf{1}\right) .
\end{aligned}
$$

Using (4.13b), the matrix $\boldsymbol{X}$ can be expressed as $\boldsymbol{X}=\boldsymbol{X}_{0}+\boldsymbol{x} \boldsymbol{x}^{T}$, where $\boldsymbol{X}_{0} \succeq \mathbf{0}$. Substituting this equation in (A.5),

$$
\begin{aligned}
\operatorname{Tr}\left(\boldsymbol{F}_{m} \boldsymbol{\Psi}\right) & =4 \sigma^{2}+\operatorname{Tr}\left(\boldsymbol{D}_{m} \boldsymbol{X}_{0}+\boldsymbol{x}^{T} \boldsymbol{D}_{m} \boldsymbol{x}+2 \boldsymbol{x}^{T} \boldsymbol{D}_{m} \mathbf{1}+\mathbf{1}^{T} \boldsymbol{D}_{m} \mathbf{1}\right) \\
& =4 \sigma^{2}+\operatorname{Tr}\left(\boldsymbol{D}_{m} \boldsymbol{X}_{0}\right)+(\boldsymbol{x}+\mathbf{1})^{T} \boldsymbol{D}_{m}(\boldsymbol{x}+\mathbf{1})
\end{aligned}
$$

The statement of the lemma follows from noting that $\sigma^{2}>0$ and that $\boldsymbol{X}_{0}$ and $\boldsymbol{D}_{m}$ are PSD for all $m$. The latter argument follows from the fact that, since $\boldsymbol{D}_{m}$ is symmetric and PSD, it can be expressed as $\boldsymbol{D}_{m}=\boldsymbol{R}_{m}^{T} \boldsymbol{R}_{m}$ using the Cholesky factorization [71, Section 10.3], where $\boldsymbol{R}_{m}$ is an upper-triangular matrix. Then

$$
\operatorname{Tr}\left(\boldsymbol{D}_{m} \boldsymbol{X}_{0}\right)=\operatorname{Tr}\left(\boldsymbol{R}_{m}^{T} \boldsymbol{R}_{m} \boldsymbol{X}_{0}\right)=\operatorname{Tr}\left(\boldsymbol{R}_{m} \boldsymbol{X}_{0} \boldsymbol{R}_{m}^{T}\right) \geq 0
$$




\section{Bibliography}

[1] A. Saleh, A. Rustako, and R. Roman, "Distributed antennas for indoor radio communications," IEEE Trans. Commun., vol. 35, no. 12, pp. 1245-1251, Dec. 1987.

[2] S. V. Hanly and P. Whiting, "Information-theoretic capacity of multi-receiver networks," Telecommunication Systems, vol. 1, no. 1, pp. 1-42, Dec. 1993.

[3] A. Wyner, "Shannon-theoretic approach to a gaussian cellular multiple-access channel," IEEE Trans. Inf. Theory, vol. 40, no. 6, pp. 1713-1727, Nov. 1994.

[4] S. Shamai and B. Zaidel, "Enhancing the cellular downlink capacity via coprocessing at the transmitting end," in Proc. IEEE Veh. Technol. Conf., vol. 3, 2001, pp. 1745-1749.

[5] G. Boudreau, J. Panicker, N. Guo, R. Chang, N. Wang, and S. Vrzic, "Interference coordination and cancellation for $4 \mathrm{G}$ networks," IEEE Commun. Mag., vol. 47, no. 4, pp. 74-81, Apr. 2009.

[6] M. Sawahashi, Y. Kishiyama, A. Morimoto, D. Nishikawa, and M. Tanno, "Coordinated multipoint transmission/reception techniques for LTE-advanced," IEEE Wireless Commun. Mag., vol. 17, no. 3, pp. 26-34, Jun. 2010.

[7] A. Ghosh, R. Ratasuk, B. Mondal, N. Mangalvedhe, and T. Thomas, "LTEAdvanced: next-generation wireless broadband technology," IEEE Wireless Commun. Mag., vol. 17, no. 3, pp. 10-22, Jun. 2010.

[8] 3rd Generation Partnership Project (3GPP), "Overview of 3GPP Release 11 V0.0.7," 3GPP, Tech. Rep., Jun. 2011. [Online]. Available: http: //www.3gpp.org/ftp/Information/WORK_PLAN/Description_Releases/

[9] _-, "Technical Specification Group Radio Access Network; Evolved Universal Terrestrial Radio Access (E-UTRA); Further advancements for E-UTRA physical layer aspects," 3GPP, TR 36.814, Mar. 2010. [Online]. Available: http://www.3gpp.org/ftp/Specs/archive/36_series/36.814/

[10] H. Yanikomeroglu and E. Sousa, "CDMA distributed antenna system for indoor wireless communications," in Proc. IEEE Int. Conf. on Universal Personal Commun., vol. 2, Oct. 1993, pp. 990-994. 
[11] - "CDMA sectorized distributed antenna system," in Proc. IEEE 5th Int. Symp. Spread Spectrum Technıques and Applıcatıons, vol. 3, Sep. 1998, pp. 792797.

[12] — - "Power control and number of antenna elements in CDMA distributed antenna systems," in Proc. IEEE Int. Conf. Commun., vol. 2, Jun. 1998, pp. $1040-1045$.

[13] A. Obaid and H. Yanikomeroglu, "Reverse-link power control in CDMA distributed antenna systems," in Proc. IEEE Wireless Commun. Networkıng Conf., vol. 2, 2000, pp. 608-612.

[14] H. Yanikomeroglu and E. Sousa, "Antenna gain against interference in cdma macrodiversity systems," IEEE Trans. Commun., vol. 50, no. 8, pp. 1356-1371, Aug. 2002.

[15] W. Roh and A. Paulraj, "Outage performance of the distributed antenna systems in a composite fading channel," in Proc. IEEE 56th Veh. Technol. Conf., vol. 3, Sep. 2002, pp. 1520-1524.

[16] _- "MIMO channel capacity for the distributed antenna systems," in Proc. IEEE 56th Veh. Technol. Conf., vol. 2, Sep. 2002, pp. 706-709.

[17] L. Xiao, L. Dai, H. Zhuang, S. Zhou, and Y. Yao, "Information-theoretic capacity analysis in MIMO distributed antenna systems," in Proc. IEEE 57th Veh. Technol. Conf., vol. 1, Apr. 2003, pp. 779-782.

[18] T. Wang, Y. Wang, K. Sun, and Z. Chen, "On the performance of downlink transmission for distributed antenna systems with multi-antenna arrays," in Proc. IEEE 70th Veh. Technol. Conf., Sep. 2009.

[19] L. Ling, T. Wang, Y. Wang, and C. Shi, "Schemes of power allocation and antenna port selection in OFDM distributed antenna systems," in Proc. IEEE 72nd Veh. Technol. Conf., Sep. 2010.

[20] X.-H. You, D.-M. Wang, B. Sheng, X.-Q. Gao, X.-S. Zhao, and M. Chen, "Cooperative distributed antenna systems for mobile communications," IEEE Wireless Commun. Mag., vol. 17, no. 3, pp. 35-43, Jun. 2010.

[21] H. Zhu, S. Karachontzitis, and D. Toumpakaris, "Low-complexity resource allocation and its application to distributed antenna systems," IEEE Wireless Commun. Mag., vol. 17, no. 3, pp. 44-50, Jun. 2010.

[22] W. Liu, S. Ng, and L. Hanzo, "Multicell cooperation based SVD assisted multiuser MIMO transmission," in Proc. IEEE Veh. Technol. Conf., Apr. 2009.

[23] H. Dahrouj and W. Yu, "Coordinated beamforming for the multicell multiantenna wireless system," IEEE Trans. Wireless Commun., vol. 9, no. 5, pp. 1748-1759, May 2010. 
[24] L. Venturino, N. Prasad, and X. Wang, "Coordinated scheduling and power allocation in downlink multicell OFDMA networks," IEEE Trans. Veh. Technol., vol. 58, no. 6, pp. 2835-2848, Jul. 2009.

[25] H. Huang and M. Trivellato, "Performance of multiuser MIMO and network coordination in downlink cellular networks," in Proc. Int. Symp. on Modeling and Optimization in Mobile, Ad Hoc, and Wireless Networks and Workshops, Apr. 2008, pp. 85-90.

[26] J. Zhang, R. Chen, J. Andrews, A. Ghosh, and R. Heath, "Networked MIMO with clustered linear precoding," IEEE Trans. Wireless Commun., vol. 8, no. 4, pp. 1910-1921, Apr. 2009.

[27] E. Björnson, R. Zakhour, D. Gesbert, and B. Ottersten, "Cooperative multicell precoding: Rate region characterization and distributed strategies with instantaneous and statistical CSI," IEEE Trans. Signal Process., vol. 58, no. 8, pp. 4298-4310, 2010.

[28] R. Zhang and L. Hanzo, "Joint and distributed linear precoding for centralised and decentralised multicell processing," in Proc. IEEE Veh. Technol. Conf., Sep. 2010 .

[29] G. Foschini, K. Karakayali, and R. Valenzuela, "Coordinating multiple antenna cellular networks to achieve enormous spectral efficiency," IEE Proc.-Commun., vol. 153, no. 4, pp. 548-555, Aug. 2006.

[30] S. Jing, D. Tse, J. Soriaga, J. Hou, J. Smee, and R. Padovani, "Multicell downlink capacity with coordinated processing," EURASIP J. Wirel. Commun. Netw., vol. 2008, pp. 18:1-18:19, Jan. 2008.

[31] S. Parkvall, E. Dahlman, A. Furuskär, Y. Jading, M. Olsson, S. Wänstedt, and K. Zangi, "LTE-Advanced - evolving LTE towards IMT-Advanced," in Proc. IEEE Veh. Technol. Conf., Sep. 2008.

[32] Y. Liang, A. Goldsmith, G. Foschini, R. Valenzuela, and D. Chizhik, "Evolution of base stations in cellular networks: Denser deployment versus coordination," in Proc. IEEE Int. Conf. Commun., May 2008, pp. 4128-4132.

[33] D. Gesbert, S. Hanly, H. Huang, S. Shamai Shitz, O. Simeone, and W. Yu, "Multi-cell mimo cooperative networks: A new look at interference," IEEE J. Sel. Areas Commun., vol. 28, no. 9, pp. 1380-1408, Dec. 2010.

[34] M. Karakayali, G. Foschini, and R. Valenzuela, "Network coordination for spectrally efficient communications in cellular systems," IEEE Wireless Commun. Mag., vol. 13, no. 4, pp. 56-61, Aug. 2006.

[35] E. Telatar, "Capacity of multi-antenna gaussian channels," European Transactions on Telecommunications, vol. 10, no. 6, pp. 585-595, Nov. 1999. 
[36] M. Costa, "Writing on dirty paper," IEEE Trans. Inf. Theory, vol. 29, no. 3, pp. 439-441, May 1983.

[37] H. Weingarten, Y. Steinberg, and S. Shamai, "The capacity region of the gaussian multiple-input multiple-output broadcast channel," IEEE Trans. Inf. Theory, vol. 52, no. 9, pp. 3936-3964, Sep. 2006.

[38] G. Caire and S. Shamal, "On the achievable throughput of a multiantenna gaussian broadcast channel," IEEE Trans. Inf. Theory, vol. 49, no. 7, pp. 1691-1706, Jul. 2003.

[39] Q. Spencer, A. Swindlehurst, and M. Haardt, "Zero-forcing methods for downlink spatial multiplexing in multiuser MIMO channels," IEEE Trans. Srgnal Process., vol. 52, no. 2, pp. 461-471, Feb. 2004.

[40] M. Karakayali, G. Foschini, R. Valenzuela, and R. Yates, "On the maximum common rate achievable in a coordinated network," in Proc. IEEE Int. Conf. Commun., vol. 9, Jun. 2006, pp. 4333-4338.

[41] A. Dabbagh and D. Love, "Precoding for multiple antenna gaussian broadcast channels with successive zero-forcing," IEEE Trans. Signal Process., vol. 55, no. 7, pp. 3837-3850, Jul. 2007.

[42] M. Maddah-Ali, M. Sadrabadi, and A. Khandani, "Broadcast in MIMO systems based on a generalized QR decomposition: Signaling and performance analysis," IEEE Trans. Inf. Theory, vol. 54, no. 3, pp. 1124-1138, Mar. 2008.

[43] L. Sun and M. McKay, "Eigenmode transmission for the MIMO broadcast channel with semi-orthogonal user selection," in Proc. IEEE Globecom, Nov. 2009, pp. 2212-2217.

[44] W. Yu and J. Cioffi, "Trellis precoding for the broadcast channel," in Proc. IEEE Globecom, vol. 2, 2001, pp. 1344-1348.

[45] R. Etkin, D. Tse, and H. Wang, "Gaussian interference channel capacity to within one bit," IEEE Trans. Inf. Theory, vol. 54, no. 12, pp. 5534-5562, Dec. 2008 .

[46] T. Moon and W. Stirling, Mathematıcal Methods and Algorithms for Sıgnal Processing. Upper Saddle River, NJ: Prentice Hall, 2000.

[47] R. Zhang, "Cooperative multi-cell block diagonalization with per-base-station power constraints," IEEE J. Sel. Areas Commun., vol. 28, no. 9, pp. 1435-1445, Dec. 2010.

[48] A. Tolli, M. Codreanu, and M. Juntti, "Linear cooperative multiuser MIMO transceiver design with per BS power constraints," in Proc. IEEE Int. Conf. Commun., Jun. 2007, pp. 4991-4996. 
[49] S. Shi, M. Schubert, N. Vucic, and H. Boche, "MMSE optimization with perbase-station power constraints for network MIMO systems," in Proc. IEEE Int. Conf. Commun., May 2008, pp. 4106-4110.

[50] Y. Hadisusanto, L. Thiele, and V. Jungnickel, "Distributed base station cooperation via block-diagonalization and dual-decomposition," in Proc. IEEE Globecom, Dec. 2008.

[51] D. Tse and P. Viswanath, Fundamentals of Wireless Communicatıons. New York: Cambridge University Press, 2005.

[52] R. A. Horn and C. R. Johnson, Matrux Analysıs. New York, NY: Cambridge University Press, 1985.

[53] J. Park, E. Song, and W. Sung, "Capacity analysis for distributed antenna systems using cooperative transmission schemes in fading channels," IEEE Trans. Wireless Commun., vol. 8, no. 2, pp. 586-592, Feb. 2009.

[54] W. Choi and J. Andrews, "Downlink performance and capacity of distributed antenna systems in a multicell environment," IEEE Trans. Wireless Commun., vol. 6, no. 1, pp. 69-73, Jan. 2007.

[55] X. Wang, P. Zhu, and M. Chen, "Antenna location design for generalized distributed antenna systems," IEEE Trans. Commun., vol. 13, no. 5, pp. 315-317, May 2009.

[56] T. Zhang, C. Zhang, L. Cuthbert, and Y. Chen, "Energy efficient antenna de-ployment design scheme in distributed antenna systems," in Proc. IEEE Veh. Technol. Conf., Sep. 2010.

[57] International Telecommunication Union (ITU), "Guidelines for evaluation of radio interface technologies for IMT-Advanced," ITU-R, TR M.2135-1, Dec. 2009. [Online]. Available: http://www.itu.int/pub/R-REP-M.2135-1-2009

[58] 3rd Generation Partnership Project (3GPP), "Evolved Universal Terrestrial Radio Access (E-UTRA); Radio Frequency (RF) system scenarios," 3GPP, TR 36.942, Dec. 2010. [Online]. Available: http://www.3gpp.org/ftp/Specs/ html-info/36942.htm

[59] L. Vandenberghe and S. Boyd, "Semidefinite programming," SIAM Rev., vol. 38, no. 1, pp. 49 95, Mar. 1996.

[60] N. Sidiropoulos, T. Davidson, and Z.-Q. Luo, "Transmit beamforming for physical-layer multicasting," IEEE Trans. Sıgnal Process., vol. 54, no. 6, pp. 2239-2251, 2006.

[61] S. Boyd and L. Vandenberghe, Convex Optımızatıon. Cambridge, U.K.: Cambridge Univ. Press, 2004. 
[62] Z.-Q. Luo, W.-K. Ma, A.-C. So, Y. Ye, and S. Zhang, "Semidefinite relaxation of quadratic optimization problems," IEEE Srgnal Process. Mag., vol. 27, no. 3, pp. 20-34, May 2010.

[63] W.-K. Ma, T. Davidson, K. M. Wong, Z.-Q. Luo, and P.-C. Ching, "Quasimaximum-likelihood multiuser detection using semi-definite relaxation with application to synchronous CDMA," IEEE Trans. Signal Process., vol. 50, no. 4, pp. 912-922, Apr. 2002.

[64] M. Goemans and D. Williamson, "Improved approximation algorithms for maximum cut and satisfiability problem using semi-definite programming," J. $A C M$, vol. 42, no. 6, pp. 1115-1145, Nov. 1995.

[65] E. Karipidis, N. Sidiropoulos, and Z.-Q. Luo, "Quality of service and max-min fair transmit beamforming to multiple cochannel multicast groups," IEEE Trans. Signal Process., vol. 56, no. 3, pp. 1268-1279, 2008.

[66] T.-H. Chang, Z.-Q. Luo, and C.-Y. Chi, "Approximation bounds for semidefinite relaxation of max-min-fair multicast transmit beamforming problem," IEEE Trans. Signal Process., vol. 56, no. 8, pp. 3932-3943, 2008.

[67] G. H. Golub and C. F. van Loan, Matrıx Computatıons, 3rd ed. Baltimore, MD: The Johns Hopkins University Press, 1996.

[68] M. Grant and S. Boyd, "CVX: Matlab software for disciplined convex programming, version 1.21," http://cvxr.com/cvx, Jan. 2011.

[69] Z.-Q. Luo, T. Davidson, G. Giannakis, and K. M. Wong, "Transceiver optimization for block-based multiple access through ISI channels," IEEE Trans. Signal Process., vol. 52, no. 4, pp. 1037-1052, Apr. 2004.

[70] C. Helmberg, F. Rendl, R. J. Vanderbei, and H. Wolkowicz, "An interior-point method for semidefinite programming," SIAM J. Optım., vol. 6, no. 2, pp. 342$361,1996$.

[71] N. J. Higham, Accuracy and Stabultty of Numerncal Algorithms. Philadelphia, PA: Society for Industrial and Applied Mathematics, 2002. 